\title{
Double crossed products of locally compact quantum groups
}

\author{
Journal of the Institute of Mathematics of Jussieu 4 (2005), 135-173.
}

By SaAd BaAJ And StefaAn Vaes

\begin{abstract}
Laboratoire de Mathématiques Pures; Université Blaise Pascal; Bâtiment de Mathématiques; F-63177 Aubière Cedex (France) e-mail: Saad.Baaj@math.univ-bpclermont.fr

Institut de Mathématiques de Jussieu; Algèbres d'Opérateurs et Représentations; 175, rue du Chevaleret; F-75013 Paris (France) e-mail: vaes@math.jussieu.fr
\end{abstract}

\begin{abstract}
For a matched pair of locally compact quantum groups, we construct the double crossed product as a locally compact quantum group. This construction generalizes Drinfeld's quantum double construction. We study the modular theory and the $\mathrm{C}^{*}$-algebraic properties of these double crossed products, as well as several links between double crossed products and bicrossed products. In an appendix, we study the Radon-Nikodym derivative of a weight under a quantum group action, following Yamanouchi and obtain, as a corollary, a new characterization of closed quantum subgroups.
\end{abstract}

\section{Introduction}

In this paper, we study the so-called double crossed product construction in the framework of locally compact quantum groups, generalizing Drinfeld's quantum double construction and bringing it into the topological setting.

The history of locally compact quantum groups goes back to the 1960's, when G. Kac tried to answer the following basic question: is it possible to unify locally compact (non-abelian) groups and their dual objects into one theory? A positive answer was given by Kac, Kac \& Vainerman and independently, by Enock \& Schwartz. They defined what Enock \& Schwartz called Kac algebras, see [5] for an overview. In the 1980 's, several new examples of objects certainly to be considered as quantum groups, were constructed and were shown not to be Kac algebras. Henceforth, many efforts were made to enlarge the definition of Kac algebras to include these new examples and in particular, to include Woronowicz' compact quantum groups, see $[18,19]$. Many important contributions have been made by Skandalis \& the first author [2], Woronowicz [20], Masuda \& Nakagami [11] and Van Daele [17]. Finally, the theory of locally compact quantum groups was proposed by Kustermans and the second author in [8, 9].

One of the most celebrated constructions in the early, algebraic theory of quantum groups is Drinfeld's quantum double construction [4]. More generally, Majid [10] defined the double crossed product for mutually matched Hopf algebras, yielding Drinfeld's quantum double when a Hopf algebra is matched with its dual in the canonical way.

It is a natural idea to perform analogous constructions in the topological framework of locally compact (l.c.) quantum groups. In fact, the double crossed product construction was developed for multiplicative unitaries in [2] (it is called $Z$-produit tensoriel, there). Nevertheless, some extra conditions had to be imposed in order to obtain the double crossed product and it was not clear if they were satisfied for all matchings of two multiplicative unitaries.

In this paper, we show that any matching of l.c. quantum groups allows to perform the double crossed product construction and to obtain again a l.c. quantum group. In particular, the quantum double of a l.c. quantum group is again a l.c. quantum group: a result already shown for Woronowicz algebras by Yamanouchi [23].

2000 Mathematics Subject Classification: Primary 46L89, Secondary 46L65.

Keywords and phrases: locally compact quantum groups, matched pair, Radon-Nikodym derivative, quantum subgroup. 
The notion of a matching of l.c. quantum groups was developed by Vainerman and the second author in [15] and the bicrossed product was constructed. We explain all these different constructions in a more detailed way for a matching of ordinary l.c. groups. One says that a pair of l.c. groups $G_{1}, G_{2}$ is a matched pair, if there exists a large group $G$, such that $G_{1}, G_{2}$ are closed subgroups, satisfying $G_{1} \cap G_{2}=\{e\}$ and such that the complement of $G_{1} G_{2}$ has Haar measure zero in $G$. In fact, this allows to define an action $\left(\alpha_{g}\right)_{g \in G_{1}}$ of $G_{1}$ by (almost everywhere defined) transformations of the measure space $G_{2}$ (not by automorphisms) and an action $\left(\beta_{s}\right)_{s \in G_{2}}$ of $G_{2}$ by transformations of the measure space $G_{1}$ such that $\alpha_{g}(s) \beta_{s}(g)=g s$ almost everywhere. Conversely, one can say that $G_{1}, G_{2}$ are matched when they act on each other in such a compatible way. Such a compatible pair of actions allows to reconstruct the l.c. group $G$, this is the double crossed product, and to construct a l.c. quantum group with underlying von Neumann algebra $G_{1 \alpha} \ltimes L^{\infty}\left(G_{2}\right)$, this is the bicrossed product.

In the quantum setting, a matching of two l.c. quantum groups was defined in [15] as a compatible pair of actions of the two quantum groups on each other. So, it is a natural question if one can find the double crossed product, i.e. a big l.c. quantum group such that the matched quantum groups are closed quantum subgroups and such that the matching is determined by this inclusion in the double crossed product, as above by the equation $\alpha_{g}(s) \beta_{s}(g)=g s$. In our main result, Theorem 5.3 below, we show that there always exists a double crossed product quantum group and we calculate its structural ingredients. In Proposition 7.1 below, we show that the notions of a matched pair and a quantum group with two closed quantum subgroups are in bijective correspondence.

In [3], Skandalis and the authors studied the $\mathrm{C}^{*}$-algebraic features of the bicrossed product associated with a matched pair $G_{1}, G_{2} \subset G$ of l.c. groups. The main result was that the bicrossed product is regular if and only if $G=G_{1} G_{2}$ homeomorphically, while it is semi-regular if and only if $G_{1} G_{2}$ is an open subset of $G$. Examples of non-semi-regular l.c. quantum groups were given using examples of matched pairs $G_{1}, G_{2} \subset G$ such that $G_{1} G_{2}$ has empty interior in $G$ (but, as needed, $G_{1} G_{2}$ is of full Haar measure in $G$ ). The fact that $G=G_{1} G_{2}$ homeomorphically can be expressed in $\mathrm{C}^{*}$-algebraic terms as $C_{0}(G)=C_{0}\left(G_{1}\right) \otimes C_{0}\left(G_{2}\right)$, while the fact that $G_{1} G_{2}$ is an open subset of $G$ becomes $C_{0}\left(G_{1}\right) \otimes C_{0}\left(G_{2}\right) \subset C_{0}(G)$. In Section 9 , we study the same kind of $\mathrm{C}^{*}$-algebraic questions in the quantum setting and provide, in particular, the same kind of necessary and sufficient condition for the bicrossed product to be (semi-)regular. More specifically, regularity is equivalent with the equation $A_{\mathrm{m}}=A_{1} \otimes A_{2}$ and semi-regularity with $A_{1} \otimes A_{2} \subset A_{\mathrm{m}}$, where $A_{1}, A_{2}$ and $A_{\mathrm{m}}$ denote the $\mathrm{C}^{*}$-algebras associated with the two matched quantum groups and the double crossed product, respectively.

The structure of the paper is as follows: we first recall the necessary preliminaries in Section 2, in particular the basics of the theory of l.c. quantum groups and the main results on matchings and bicrossed products from [15]. Sections $3-5$ are the core of the paper: we define the double crossed product for a matched pair and prove that it is a l.c. quantum group. In order to do so, we have to produce the Haar weights and we will need a continuous interplay between the double crossed product and the bicrossed product, about which we already know a lot from [15]. In the short Section 6, we show how actions of double crossed products on von Neumann algebras and actions of bicrossed products are related by an intermediate von Neumann algebra in the Jones tower. In Section 7, we prove the bijective correspondence between a matched pair and a quantum group with two closed quantum subgroups in a good position with respect to each other. The example of a (generalized) quantum double construction is treated in Section 8 and we deal with the $\mathrm{C}^{*}$-algebraic features of our constructions in Section 9.

One of our major tools is the Radon-Nikodym derivative of a weight under a l.c. quantum group action, as developed originally by Yamanouchi [22]. In an appendix, Section 10, we give a simplified account of Yamanouchi's interesting results. As an application, somehow aside of the main line of our paper, we give a characterization of closed quantum subgroups as von Neumann subalgebras invariant under the comultiplication and the antipode.

Acknowledgment The authors would like to thank Georges Skandalis for many fruitful discussions on the topic of this paper. 


\section{Preliminaries}

We use the following conventions: $\otimes$ will denote, depending on the case, the tensor product of Hilbert spaces, von Neumann algebras or $\mathrm{C}^{*}$-algebras. There should be no confusion. The domain of an unbounded mapping $S$ is denoted by $D(S)$. We write $\sigma$ for the flip map on the tensor product $M \otimes M$ of two von Neumann algebras and $\Sigma$ for the flip map on the tensor product of two Hilbert spaces $H \otimes H$.

\subsection{A little bit of weight theory}

Let $\varphi$ be a normal, semi-finite, faithful (n.s.f.) weight on a von Neumann algebra $M$. Then, we write

$$
\mathcal{M}_{\varphi}^{+}=\left\{x \in M^{+} \mid \varphi(x)<\infty\right\} \quad \text { and } \quad \mathcal{N}_{\varphi}=\left\{x \in M \mid \varphi\left(x^{*} x\right)<\infty\right\} .
$$

We represent $M$ on its GNS-space $H$ and obtain a GNS-map $\Lambda: \mathcal{N}_{\varphi} \rightarrow H$. Denote by $\left(\sigma_{t}\right)$ the modular group of $\varphi$.

Suppose that $k$ is a strictly positive, self-adjoint operator affiliated with $M$ and satisfying $\sigma_{t}(k)=\lambda^{t} k$ for all $t \in \mathbb{R}$ and a number $\lambda>0$. Then, we can define a new n.s.f. weight $\varphi_{k}$ which is formally given by $\varphi_{k}(x)=\varphi\left(k^{1 / 2} x k^{1 / 2}\right)$. For a precise definition, we refer to [14]. We also get a canonical GNS-map $\Lambda_{\varphi_{k}}: \mathcal{N}_{\varphi_{k}} \rightarrow H$ for the weight $\varphi_{k}$, which is formally given by $\Lambda_{\varphi_{k}}(x)=\Lambda\left(x k^{1 / 2}\right)$.

\subsection{Locally compact quantum groups}

Our references for the theory of l.c. quantum groups are $[8,9]$.

Definition 2.1. A pair $(M, \Delta)$ is called a (von Neumann algebraic) l.c. quantum group when

- $M$ is a von Neumann algebra and $\Delta: M \rightarrow M \otimes M$ is a normal and unital $*$-homomorphism satisfying the coassociativity relation : $(\Delta \otimes \iota) \Delta=(\iota \otimes \Delta) \Delta$;

- there exist n.s.f. weights $\varphi$ and $\psi$ on $M$ such that

$-\varphi$ is left invariant in the sense that $\varphi((\omega \otimes \iota) \Delta(x))=\varphi(x) \omega(1)$ for all $x \in \mathcal{M}_{\varphi}^{+}$and $\omega \in M_{*}^{+}$,

- $\psi$ is right invariant in the sense that $\psi((\iota \otimes \omega) \Delta(x))=\psi(x) \omega(1)$ for all $x \in \mathcal{M}_{\psi}^{+}$and $\omega \in M_{*}^{+}$.

Fix such a l.c. quantum group $(M, \Delta)$.

Represent $M$ in the GNS-construction of $\varphi$ with GNS-map $\Lambda: \mathcal{N}_{\varphi} \rightarrow H$. We define a unitary $W$ on $H \otimes H$ by

$$
W^{*}(\Lambda(a) \otimes \Lambda(b))=(\Lambda \otimes \Lambda)(\Delta(b)(a \otimes 1)) \quad \text { for all } a, b \in \mathcal{N}_{\varphi} .
$$

Here, $\Lambda \otimes \Lambda$ denotes the canonical GNS-map for the tensor product weight $\varphi \otimes \varphi$. One proves that $W$ satisfies the pentagonal equation: $W_{12} W_{13} W_{23}=W_{23} W_{12}$, and we say that $W$ is a multiplicative unitary. It is the left regular representation. The von Neumann algebra $M$ is the strong closure of the algebra $\left\{(\iota \otimes \omega)(W) \mid \omega \in \mathrm{B}(H)_{*}\right\}$ and $\Delta(x)=W^{*}(1 \otimes x) W$, for all $x \in M$. Next, the l.c. quantum group $(M, \Delta)$ has an antipode $S$, which is the unique $\sigma$-strong* closed linear map from $M$ to $M$ satisfying $(\iota \otimes \omega)(W) \in D(S)$ for all $\omega \in \mathrm{B}(H)_{*}$ and $S(\iota \otimes \omega)(W)=(\iota \otimes \omega)\left(W^{*}\right)$ and such that the elements $(\iota \otimes \omega)(W)$ form a $\sigma$-strong* core for $S$. The antipode $S$ has a polar decomposition $S=R \tau_{-i / 2}$ where $R$ is an anti-automorphism of $M$ and $\left(\tau_{t}\right)$ is a strongly continuous one-parameter group of automorphisms of $M$. We call $R$ the unitary antipode and $\left(\tau_{t}\right)$ the scaling group of $(M, \Delta)$. From [8], Proposition 5.26 we know that $\sigma(R \otimes R) \Delta=\Delta R$. So $\varphi R$ is a right invariant weight on $(M, \Delta)$ and we take $\psi:=\varphi R$.

We denote by $\left(\sigma_{t}\right)$ the modular automorphism group of $\varphi$. From [8], Proposition 6.8 we get the existence of a number $\nu>0$, called the scaling constant, such that $\psi \sigma_{t}=\nu^{-t} \psi$ for all $t \in \mathbb{R}$. Hence, we get the existence of a unique positive, self-adjoint operator $\delta$ affiliated to $M$, such that $\sigma_{t}(\delta)=\nu^{t} \delta$ for all $t \in \mathbb{R}$ and $\psi=\varphi_{\delta}$, see [8], Definition 7.1. The operator $\delta$ is called the modular element of $(M, \Delta)$ and the canonically associated 
GNS-map is denoted by $\Gamma: \mathcal{N}_{\psi} \rightarrow H$. Because of the right invariance of $\psi$, we find another multiplicative unitary, denoted by $V$ and called the right regular representation:

$$
V(\Gamma(x) \otimes \Gamma(y))=(\Gamma \otimes \Gamma)(\Delta(x)(1 \otimes y)) \quad \text { for all } \quad x, y \in \mathcal{N}_{\psi} .
$$

The scaling constant can be characterized as well by the relative invariance $\varphi \tau_{t}=\nu^{-t} \varphi$.

The dual l.c. quantum group $(\hat{M}, \hat{\Delta})$ is defined in [8], Section 8. Its von Neumann algebra $\hat{M}$ is the strong closure of the algebra $\left\{(\omega \otimes \iota)(W) \mid \omega \in \mathrm{B}(H)_{*}\right\}$ and the comultiplication is given by $\hat{\Delta}(x)=\Sigma W(x \otimes 1) W^{*} \Sigma$ for all $x \in \hat{M}$. On $\hat{M}$ there exists a canonical left invariant weight $\hat{\varphi}$ with GNS-map $\hat{\Lambda}: \mathcal{N}_{\hat{\varphi}} \rightarrow H$ and the associated multiplicative unitary is denoted by $\hat{W}$. From [8], Proposition 8.16, it follows that $\hat{W}=\Sigma W^{*} \Sigma$. Since $(\hat{M}, \hat{\Delta})$ is again a l.c. quantum group, we can introduce the antipode $\hat{S}$, the unitary antipode $\hat{R}$, the scaling group $\left(\hat{\tau}_{t}\right)$ and the modular element $\hat{\delta}$ exactly as we did it for $(M, \Delta)$. Also, we can again construct the dual of $(\hat{M}, \hat{\Delta})$, starting from the left invariant weight $\hat{\varphi}$ with GNS-construction $(H, \iota, \hat{\Lambda})$. From $[8]$, Theorem 8.29 we have that the bidual l.c. quantum group $(\hat{\hat{M}}, \hat{\Delta})$ is isomorphic to $(M, \Delta)$.

We denote by $\left(\hat{\sigma}_{t}\right)$ the modular automorphism groups of the weight $\hat{\varphi}$. The modular conjugations of the weights $\varphi$ and $\hat{\varphi}$ will be denoted by $J$ and $\hat{J}$ respectively. Then it is worthwhile to mention that

$$
R(x)=\hat{J} x^{*} \hat{J} \quad \text { for all } x \in M \quad \text { and } \quad \hat{R}(y)=J y^{*} J \quad \text { for all } y \in \hat{M} .
$$

Some important relations involving $J$ and $\hat{J}$ are

$$
\hat{J} J=\nu^{i / 4} J \hat{J}, \quad W^{*}=(\hat{J} \otimes J) W(\hat{J} \otimes J), \quad V=(\hat{J} \otimes \hat{J}) \Sigma W^{*} \Sigma(\hat{J} \otimes \hat{J}) .
$$

The modular operators of $\varphi$ and $\hat{\varphi}$ are denoted by $\nabla$ and $\hat{\nabla}$, respectively. Finally, we introduce the implementation $P$ of $\left(\tau_{t}\right)$ as the unique strictly positive, self-adjoint operator on $H$ such that $P^{i t} \Lambda(x)=\nu^{t / 2} \Lambda\left(\tau_{t}(x)\right)$. We mention that $P=\hat{P}$, i.e., $P$ is as well the implementation of $\left(\hat{\tau}_{t}\right)$. For all kinds of relations between the operators on $H$ introduced so far, we refer to Proposition 2.4 in [16].

We write $\Delta^{\mathrm{op}}$ for the opposite comultiplication $\Delta^{\mathrm{op}}:=\sigma \Delta$ and observe that $\left(M, \Delta^{\mathrm{op}}\right)$ is again a l.c. quantum group.

Every l.c. quantum group has an associated $C^{*}$-algebra of 'continuous functions tending to zero at infinity' and we denote them by $A$ and $\hat{A}$ :

$$
A:=\left[(\iota \otimes \omega)(W) \mid \omega \in \mathrm{B}(H)_{*}\right], \quad \hat{A}=\left[(\omega \otimes \iota)(W) \mid \omega \in \mathrm{B}(H)_{*}\right],
$$

where we use the following notation.

Notation 2.2. When $X$ is a subset of a Banach space, we denote by $[X]$ the closed linear span of $X$. We sometimes use a notation like $[(\iota \otimes \omega)(W)]$ instead of $\left[(\iota \otimes \omega)(W) \mid \omega \in \mathrm{B}(H)_{*}\right]$, because $\omega$ will always run through $\mathrm{B}(H)_{*}$ for the appropriate Hilbert space $H$.

For any multiplicative unitary $W$ on a Hilbert space $H$, we introduce (following $[1,2]$ ) the algebra $\mathcal{C}(W)$ by the formula

$$
\mathcal{C}(W):=\left\{(\iota \otimes \omega)(\Sigma W) \mid \omega \in \mathrm{B}(H)_{*}\right\} .
$$

We say that $(M, \Delta)$ is regular (see $[2])$ if $[\mathcal{C}(W)]=\mathcal{K}(H)$, the compact operators on $H$. We say that $(M, \Delta)$ is semi-regular (see [1]) if $\mathcal{K}(H) \subset[\mathcal{C}(W)]$. From Proposition 2.6 in [3], we get that

$$
[\mathcal{C}(W)]=[J A J \hat{J} \hat{A} \hat{J}] .
$$

Not all l.c. quantum groups are semi-regular, see [3]. 


\subsection{Actions of l.c. quantum groups}

We use [13] as a reference for actions of l.c. quantum groups, but we repeat the necessary elements of the theory. Let $(M, \Delta)$ be a l.c. quantum group and $N$ a von Neumann algebra. A faithful, normal *homomorphism $\alpha: N \rightarrow M \otimes N$ is called a (left) action of $(M, \Delta)$ on $N$ if $(\Delta \otimes \iota) \alpha=(\iota \otimes \alpha) \alpha$. One can define the crossed product as

$$
M_{\alpha} \ltimes N=(\alpha(N) \cup \hat{M} \otimes 1)^{\prime \prime} \in \mathrm{B}(H) \otimes N .
$$

Given an n.s.f. weight $\theta$ on $N$, one can define the dual weight $\tilde{\theta}$ on the crossed product $M_{\alpha} \ltimes N$. Representing $N$ on the GNS-space $H_{\theta}$ of $\theta$, we get a GNS-map $\Lambda_{\theta}: \mathcal{N}_{\theta} \rightarrow H_{\theta}$. From [13], Definition 3.4 and Proposition 3.10, we get a canonical GNS-construction for $\tilde{\theta}$ on the Hilbert space $H \otimes H_{\theta}$, with GNS-map determined by

$$
(x \otimes 1) \alpha(y) \mapsto \hat{\Lambda}(x) \otimes \Lambda_{\theta}(y) \quad \text { for } \quad x \in \mathcal{N}_{\hat{\varphi}}, y \in \mathcal{N}_{\theta}
$$

and where these elements $(x \otimes 1) \alpha(y)$ span a core for the GNS-map, when we equip $M_{\alpha} \ltimes N$ with its $\sigma$-strong* topology and $H \otimes H_{\theta}$ with the norm topology.

If we denote by $J_{\theta}$ the modular conjugation of $\theta$ and by $\tilde{J}_{\theta}$ the modular conjugation of the dual weight $\tilde{\theta}$ in the canonical GNS-construction for $\tilde{\theta}$, we can define the unitary $U_{\alpha}=\tilde{J}_{\theta}\left(\hat{J} \otimes J_{\theta}\right)$, which is called the unitary implementation of $\alpha$, see [13]. It satisfies

$$
U_{\alpha} \in M \otimes \mathrm{B}\left(H_{\theta}\right), \quad(\Delta \otimes \iota)\left(U_{\alpha}\right)=U_{\alpha, 23} U_{\alpha, 13} \quad \text { and } \quad \alpha(x)=U_{\alpha}(1 \otimes x) U_{\alpha}^{*} \text { for } x \in N .
$$

Because $U_{\alpha}^{*}$ is a corepresentation of $(M, \Delta)$, we get that $U_{\alpha} \in \mathrm{M}\left(A \otimes \mathcal{K}\left(H_{\theta}\right)\right)$, where $A \subset M$ is the $\mathrm{C}^{*}$-algebra associated with $(M, \Delta)$.

If $\alpha: N \rightarrow M \otimes N$ is an action, the fixed point algebra is denoted by $N^{\alpha}$ and defined as the von Neumann subalgebra of elements $x \in N$ satisfying $\alpha(x)=1 \otimes x$.

Up to now, we dealt with left actions, but this is only a matter of convention. We can (and will) also consider right actions $\alpha: N \rightarrow N \otimes M$ of $(M, \Delta)$ on a von Neumann algebra $N$, which means that $(\alpha \otimes \iota) \alpha=(\iota \otimes \Delta) \alpha$.

\subsection{Matchings and bicrossed products of l.c. quantum groups}

We give a quick overview of some of the results of [15]. Fix two l.c. quantum groups $\left(M_{1}, \Delta_{1}\right)$ and $\left(M_{2}, \Delta_{2}\right)$.

Definition 2.3. A normal, faithful ${ }^{*}$-homomorphism $\mathrm{m}: M_{1} \otimes M_{2} \rightarrow M_{1} \otimes M_{2}$ is called a matching of $\left(M_{1}, \Delta_{1}\right)$ and $\left(M_{2}, \Delta_{2}\right)$ if

$$
\left(\Delta_{1} \otimes \iota\right) \mathrm{m}=\mathrm{m}_{23} \mathrm{~m}_{13}\left(\Delta_{1} \otimes \iota\right) \quad \text { and } \quad\left(\iota \otimes \Delta_{2}\right) \mathrm{m}=\mathrm{m}_{13} \mathrm{~m}_{12}\left(\iota \otimes \Delta_{2}\right) .
$$

We fix a matching $\mathrm{m}$ of $\left(M_{1}, \Delta_{1}\right)$ and $\left(M_{2}, \Delta_{2}\right)$. As in [15], we define

$$
\alpha: M_{2} \rightarrow M_{1} \otimes M_{2}: \alpha(x)=\mathrm{m}(1 \otimes x) \quad \text { and } \quad \beta: M_{1} \rightarrow M_{1} \otimes M_{2}: \beta(y)=\mathrm{m}(y \otimes 1) .
$$

Then, $\alpha$ is a left action of $\left(M_{1}, \Delta_{1}\right)$ on the von Neumann algebra $M_{2}$, while $\beta$ is a right action of $\left(M_{2}, \Delta_{2}^{\mathrm{op}}\right)$ on $M_{1}$. So, we can define two crossed product von Neumann algebras $M$ and $\hat{M}$ on $H_{1} \otimes H_{2}$ :

$$
M=M_{1 \alpha} \ltimes M_{2}=\left(\alpha\left(M_{2}\right) \cup \hat{M}_{1} \otimes 1\right)^{\prime \prime} \quad \text { and } \quad \hat{M}=M_{1} \rtimes_{\beta} M_{2}=\left(\beta\left(M_{1}\right) \cup 1 \otimes \hat{M}_{2}\right)^{\prime \prime} .
$$

In [15], it is proven that we can define a l.c. quantum group structure $(M, \Delta)$ on the von Neumann algebra $M$ such that $\hat{M}$ is the dual quantum group. The comultiplications on $M$ and its dual $\hat{M}$ are given by

$$
\begin{array}{ll}
\Delta \alpha=(\alpha \otimes \alpha) \Delta_{2}, & \left.(\iota \otimes \Delta)\left(W_{1} \otimes 1\right)=W_{1,14}(\iota \otimes \alpha) \beta \otimes \iota\right)\left(W_{1}\right)_{1452}, \\
\hat{\Delta} \beta=(\beta \otimes \beta) \Delta_{1}, & (\hat{\Delta} \otimes \iota)\left(1 \otimes \hat{W}_{2}\right)=(\iota \otimes(\beta \otimes \iota) \alpha)\left(\hat{W}_{2}\right)_{2345} \hat{W}_{2,25} .
\end{array}
$$

The l.c. quantum group $(M, \Delta)$ is called the bicrossed product associated with the matching $\mathrm{m}$ of $\left(M_{1}, \Delta_{1}\right)$ and $\left(M_{2}, \Delta_{2}\right)$. 
Define $\varphi$ to be the dual weight on $M$ of the weight $\varphi_{2}$ on $M_{2}$, with canonical GNS-map determined by

$$
\Lambda((a \otimes 1) \alpha(x))=\hat{\Lambda}_{1}(a) \otimes \Lambda_{2}(x) \quad \text { for } a \in \mathcal{N}_{\hat{\varphi}_{1}}, x \in \mathcal{N}_{\varphi_{2}} .
$$

Then, $\varphi$ is the left invariant weight on $(M, \Delta)$ and the left regular representation is given by

$$
W=(\alpha \otimes \iota)\left(W_{2}\right)_{124}(\iota \otimes \beta)\left(\hat{W}_{1}\right)_{134} .
$$

Observe that, on $H_{1} \otimes H_{2}$, we have the modular conjugations $J$ and $\hat{J}$ of the invariant weights $\varphi$ and $\hat{\varphi}$. So, we also have

$$
\begin{array}{ll}
U_{\alpha}=J\left(\hat{J}_{1} \otimes J_{2}\right) \in M_{1} \otimes \mathrm{B}\left(H_{2}\right), & \alpha(x)=U_{\alpha}(1 \otimes x) U_{\alpha}^{*}, \\
U_{\beta}=\hat{J}\left(J_{1} \otimes \hat{J}_{2}\right) \in \mathrm{B}\left(H_{1}\right) \otimes M_{2}, & \beta(y)=U_{\beta}(y \otimes 1) U_{\beta}^{*} .
\end{array}
$$

Notation 2.4. Throughout Sections 3 - 6, we will fix a matching $m$ of two l.c. quantum groups $\left(M_{1}, \Delta_{1}\right)$ and $\left(M_{2}, \Delta_{2}\right)$. All notations with indices 1,2 refer to ingredients of these l.c. quantum groups $\left(M_{1}, \Delta_{1}\right)$, resp. $\left(M_{2}, \Delta_{2}\right)$. All notations without indices refer to the bicrossed product quantum group $(M, \Delta)$. Finally, we will discuss an associated double crossed product and then, the index $\mathrm{m}$ will be used.

\section{Double crossed products: definition and multiplicative unitary}

The matching $\mathrm{m}$ of $\left(M_{1}, \Delta_{1}\right)$ and $\left(M_{2}, \Delta_{2}\right)$ leads naturally to a coassociative comultiplication $\Delta_{\mathrm{m}}$ in the following definition.

Definition 3.1. Define the von Neumann algebra $M_{\mathrm{m}}=M_{1} \otimes M_{2}$ and

$$
\Delta_{\mathrm{m}}: M_{\mathrm{m}} \rightarrow M_{\mathrm{m}} \otimes M_{\mathrm{m}}: \Delta_{\mathrm{m}}=(\iota \otimes \sigma \mathrm{m} \otimes \iota)\left(\Delta_{1}^{\mathrm{op}} \otimes \Delta_{2}\right) .
$$

Then, $\Delta_{\mathrm{m}}$ is a co-associative comultiplication.

One of our main objectives will be to prove that $\left(M_{\mathrm{m}}, \Delta_{\mathrm{m}}\right)$ is always a l.c. quantum group. We will explicitly calculate all the ingredients of $\left(M_{\mathrm{m}}, \Delta_{\mathrm{m}}\right)$ in terms of $\left(M_{1}, \Delta_{1}\right),\left(M_{2}, \Delta_{2}\right)$ and the bicrossed product $(M, \Delta)$.

Notation 3.2. We write $Z=J \hat{J}\left(\hat{J}_{1} J_{1} \otimes \hat{J}_{2} J_{2}\right)$.

Lemma 3.3. We have $\mathrm{m}(z)=Z z Z^{*}$ for all $z \in M_{1} \otimes M_{2}$.

Proof. For $x \in M_{1}$, we have

$$
Z(x \otimes 1) Z^{*}=\hat{J} U_{\alpha}\left(J_{1} x J_{1} \otimes 1\right) U_{\alpha}^{*} \hat{J}=\hat{J}\left(J_{1} x J_{1} \otimes 1\right) \hat{J}=U_{\beta}(x \otimes 1) U_{\beta}^{*}=\beta(x)=\mathrm{m}(x \otimes 1) .
$$

We analogously have $\mathrm{m}(1 \otimes x)=Z(1 \otimes x) Z^{*}$ for $x \in M_{2}$.

It is easy to define a unitary implementing the comultiplication $\Delta_{\mathrm{m}}$, but it is less easy to prove that this unitary is multiplicative.

Definition 3.4. Define on $H_{1} \otimes H_{2} \otimes H_{1} \otimes H_{2}$, the unitary

$$
W_{\mathrm{m}}=\left(\Sigma V_{1}^{*} \Sigma\right)_{13} Z_{34}^{*} W_{2,24} Z_{34} .
$$

Proposition 3.5. The unitary $W_{\mathrm{m}}$ is multiplicative and $\Delta_{\mathrm{m}}(z)=W_{\mathrm{m}}^{*}(1 \otimes 1 \otimes z) W_{\mathrm{m}}$ for all $z \in M_{\mathrm{m}}$. Moreover, we have $(\mathrm{m} \otimes \iota \otimes \iota)\left(W_{\mathrm{m}}\right)=Z_{34}^{*} W_{2,24} Z_{34}\left(\Sigma V_{1}^{*} \Sigma\right)_{13}$. 
Proof. Let $z \in M_{\mathrm{m}}$. Then,

$$
\begin{aligned}
W_{\mathrm{m}}^{*}(1 \otimes 1 \otimes z) W_{\mathrm{m}} & =Z_{34}^{*} W_{2,24}^{*} Z_{34}\left(\Delta_{1}^{\mathrm{op}} \otimes \iota\right)(z)_{134} Z_{34}^{*} W_{2,24} Z_{34} \\
& =Z_{34}^{*}\left(\left(\iota \otimes \iota \otimes \Delta_{2}\right)(\iota \otimes \mathrm{m})\left(\Delta_{1}^{\mathrm{op}} \otimes \iota\right)(z)\right)_{1324} Z_{34}=\Delta_{\mathrm{m}}(z) .
\end{aligned}
$$

We now prove that $W_{\mathrm{m}}$ is a multiplicative unitary.

Because the bicrossed product is a l.c. quantum group, its multiplicative unitary $W$ satisfies $(\Sigma(1 \otimes J \hat{J}) W)^{3} \in$ $\mathbb{C}$ (we can apply Proposition 6.9 of [2]). In essentially the same way as in the first paragraph of the proof of Proposition 8.14 of [2], it follows that $\left(\Sigma(1 \otimes \mathcal{U}) W_{\mathrm{m}}\right)^{3} \in \mathbb{C}$, where $\mathcal{U}=\left(J_{1} \hat{J}_{1} \otimes J_{2} \hat{J}_{2}\right) Z$. Define $N:=\left(\hat{M}_{1}^{\prime} \otimes 1 \cup Z^{*}\left(1 \otimes \hat{M}_{2}\right) Z\right)^{\prime \prime}$. We claim that $\mathcal{U} N \mathcal{U}^{*} \subset N^{\prime}$. Clearly, $\mathcal{U} Z^{*}\left(1 \otimes \hat{M}_{2}\right) Z \mathcal{U}^{*}=1 \otimes \hat{M}_{2}^{\prime}$ and this commutes with $\hat{M}_{1}^{\prime} \otimes 1$. To prove that the commutator $\left[1 \otimes \hat{M}_{2}^{\prime}, Z^{*}\left(1 \otimes \hat{M}_{2}\right) Z\right]=\{0\}$, it suffices to check that $\left[1 \otimes \hat{M}_{2}, J \hat{J}\left(1 \otimes \hat{M}_{2}\right) \hat{J} J\right]=\{0\}$. We know this, because $1 \otimes \hat{M}_{2} \subset \hat{M}$, the dual bicrossed product. Observing that, up to a scalar, $\mathcal{U}=Z^{*}\left(J_{1} \hat{J}_{1} \otimes J_{2} \hat{J}_{2}\right)$, we check in an analogous way that $\mathcal{U}\left(\hat{M}_{1}^{\prime} \otimes 1\right) \mathcal{U}^{*} \subset N^{\prime}$ and this proves our claim. Because, $\left(\Sigma(1 \otimes \mathcal{U}) W_{\mathrm{m}}\right)^{3} \in \mathbb{C}$, we get

$$
\Sigma \hat{V}_{\mathrm{m}}^{*} \hat{W}_{\mathrm{m}}^{*}(1 \otimes \mathcal{U}) W_{\mathrm{m}}(\mathcal{U} \otimes \mathcal{U}) \in \mathbb{C},
$$

where $\hat{W}_{\mathrm{m}}=\Sigma W_{\mathrm{m}}^{*} \Sigma$ and $\hat{V}_{\mathrm{m}}=\left(\mathcal{U}^{*} \otimes 1\right) W_{\mathrm{m}}^{*}(\mathcal{U} \otimes 1)$. From our claim, it follows that $(1 \otimes \mathcal{U}) W_{\mathrm{m}}(\mathcal{U} \otimes \mathcal{U}) \in$ $\mathrm{B}\left(H_{1} \otimes H_{2}\right) \otimes N^{\prime}$ and hence, we get

$$
\hat{W}_{\mathrm{m}}^{*}(1 \otimes 1 \otimes z) \hat{W}_{\mathrm{m}}=\hat{V}_{\mathrm{m}}(z \otimes 1 \otimes 1) \hat{V}_{\mathrm{m}}^{*} \quad \text { for all } z \in N .
$$

So, for $x \in \hat{M}_{2}$,

$$
\begin{aligned}
\hat{W}_{\mathrm{m}}^{*}\left(1 \otimes 1 \otimes Z^{*}(1 \otimes x) Z\right) \hat{W}_{\mathrm{m}} & =\hat{V}_{\mathrm{m}}\left(Z^{*}(1 \otimes x) Z \otimes 1 \otimes 1\right) \hat{V}_{\mathrm{m}}^{*} \\
& =\left(\mathcal{U}^{*} \otimes Z^{*}\right) W_{2,24}^{*}\left(1 \otimes \hat{J}_{2} J_{2} x J_{2} \hat{J}_{2} \otimes 1 \otimes 1\right) W_{2,24}(\mathcal{U} \otimes Z) \\
& =\left(Z^{*} \otimes Z^{*}\right)\left(\hat{\Delta}_{2}(x)\right)_{24}(Z \otimes Z) .
\end{aligned}
$$

We conclude that $W_{\mathrm{m}}\left(Z^{*}(1 \otimes x) Z \otimes 1 \otimes 1\right) W_{\mathrm{m}}^{*}=\left(Z^{*} \otimes Z^{*}\right)\left(\hat{\Delta}_{2}^{\mathrm{op}}(x)\right)_{24}(Z \otimes Z)$. It is easy to check that $W_{\mathrm{m}}(y \otimes 1 \otimes 1 \otimes 1) W_{\mathrm{m}}^{*}=\hat{\Delta}_{1}^{\prime \mathrm{op}}(y)_{13}$ for all $y \in \hat{M}_{1}^{\prime}$, where $\hat{\Delta}_{1}^{\prime}$ is the natural comultiplication on the commutant $\hat{M}_{1}^{\prime}$ given by $\hat{\Delta}_{1}^{\prime}(y)=V_{1}^{*}(1 \otimes y) V_{1}$ for $y \in \hat{M}_{1}^{\prime}$. Then, we check that

$$
W_{\mathrm{m} 3456} W_{\mathrm{m} 1234} W_{\mathrm{m} 3456}^{*}=\left(\Sigma V_{1}^{*} \Sigma\right)_{13}\left(\Sigma V_{1}^{*} \Sigma\right)_{15} Z_{34}^{*} Z_{56}^{*} W_{2,24} W_{2,26} Z_{34} Z_{56}=W_{\mathrm{m} 1234} W_{\mathrm{m} 1256} .
$$

Hence, $W_{\mathrm{m}}$ is a multiplicative unitary. Then also

$$
\left(\Delta_{\mathrm{m}} \otimes \iota \otimes \iota\right)\left(W_{\mathrm{m}}\right)=W_{\mathrm{m} 1256} W_{\mathrm{m} 3456}
$$

and a small calculation yields the formula $(\mathrm{m} \otimes \iota \otimes \iota)\left(W_{\mathrm{m}}\right)=Z_{34}^{*} W_{2,24} Z_{34}\left(\Sigma V_{1}^{*} \Sigma\right)_{13}$.

In order to prove later that $\left(M_{\mathrm{m}}, \Delta_{\mathrm{m}}\right)$ is a l.c. quantum group, we need the following remarkable lemma.

Lemma 3.6. The following holds.

a) $\mathrm{m}$ is $a^{*}$-isomorphism,

b) $\mathrm{m}\left(\tau_{-t}^{1} \otimes \tau_{t}^{2}\right)=\left(\tau_{-t}^{1} \otimes \tau_{t}^{2}\right) \mathrm{m}$ for all $t \in \mathbb{R}$,

c) $\mathrm{m}\left(R_{1} \otimes R_{2}\right)=\left(R_{1} \otimes R_{2}\right) \mathrm{m}^{-1}$.

If we write $\eta_{t}=\tau_{-t}^{1} \otimes \tau_{t}^{2}$, then the elements $(\iota \otimes \iota \otimes \omega)\left(W_{\mathrm{m}}\right), \omega \in \mathrm{B}\left(H_{1} \otimes H_{2}\right)_{*}$ form a core for $\eta_{-i / 2}$ and

$$
\left(R_{1} \otimes R_{2}\right) \mathrm{m} \eta_{-i / 2}(\iota \otimes \iota \otimes \omega)\left(W_{\mathrm{m}}\right)=(\iota \otimes \iota \otimes \omega)\left(W_{\mathrm{m}}^{*}\right) .
$$


Proof. If $\omega=\omega_{\xi, \eta}, \xi, \eta \in H_{1} \otimes H_{2}$, we take a basis $\left(e_{i}\right)_{i \in I}$ for $H_{1} \otimes H_{2}$ and get

$$
(\iota \otimes \iota \otimes \omega)\left(W_{\mathrm{m}}\right)=\sum_{i \in I}\left(\iota \otimes \omega_{e_{i}, \eta}\right)\left(\left(\Sigma V_{1}^{*} \Sigma\right)_{12}\right) \otimes\left(\iota \otimes \omega_{\xi, e_{i}}\right)\left(Z_{23}^{*} W_{2,13} Z_{23}\right) .
$$

Using the closedness of $\eta_{-i / 2}$, it is easy to conclude that $(\iota \otimes \iota \otimes \omega)\left(W_{\mathrm{m}}\right) \in D\left(\eta_{-i / 2}\right)$ and

$$
\left(R_{1} \otimes R_{2}\right) \eta_{-i / 2}(\iota \otimes \iota \otimes \omega)\left(W_{\mathrm{m}}\right)=(\iota \otimes \iota \otimes \omega)\left(\left(\Sigma V_{1} \Sigma\right)_{13} Z_{34}^{*} W_{2,24}^{*} Z_{34}\right),
$$

for all $\omega \in \mathrm{B}\left(H_{1} \otimes H_{2}\right)_{*}$. We claim that the elements $(\iota \otimes \iota \otimes \omega)\left(W_{\mathrm{m}}\right)$ provide a core for $\eta_{-i / 2}$. Denote by $D_{0}$ the domain of the closure of the restriction of $\eta_{-i / 2}$ to these elements. We have to prove that $D_{0}=D\left(\eta_{-i / 2}\right)$. Taking $\omega$ of the form $(x \otimes 1) Z^{*} \omega(1 \otimes a)$ for $x \in \mathrm{B}\left(H_{1}\right)$ and $a \in M_{2}$, we observe that

$$
z:=(\iota \otimes \iota \otimes \omega)\left(\left(\Sigma V_{1}^{*} \Sigma\right)_{13} Z_{34}^{*}(\alpha(a)(x \otimes 1))_{34} W_{2,24}\right) \in D_{0}
$$

and

$$
\left(R_{1} \otimes R_{2}\right) \eta_{-i / 2}(z)=(\iota \otimes \iota \otimes \omega)\left(\left(\Sigma V_{1} \Sigma\right)_{13} Z_{34}^{*}(\alpha(a)(x \otimes 1))_{34} W_{2,24}^{*}\right) .
$$

Any element of $\mathrm{B}\left(H_{1}\right) \otimes M_{2}$ can be approximated in the strong* topology by a bounded net of elements in $\alpha\left(M_{2}\right)\left(\mathrm{B}\left(H_{1}\right) \otimes 1\right)$ and in particular the element $1 \otimes b$ for $b \in M_{2}$. So, we find that for all $b \in M_{2}$ and $\omega \in \mathrm{B}\left(H_{1} \otimes H_{2}\right)_{*}$,

$$
z:=(\iota \otimes \iota \otimes \omega)\left(\left(\Sigma V_{1}^{*} \Sigma\right)_{13} Z_{34}^{*}\left((1 \otimes b) W_{2}\right)_{24}\right) \in D_{0}
$$

and

$$
\left(R_{1} \otimes R_{2}\right) \eta_{-i / 2}(z)=(\iota \otimes \iota \otimes \omega)\left(\left(\Sigma V_{1} \Sigma\right)_{13} Z_{34}^{*}\left((1 \otimes b) W_{2}^{*}\right)_{24}\right) .
$$

Taking in the previous formula $\omega$ of the form $(1 \otimes x) \omega$, with $x \in \hat{M}_{2}^{\prime}$ and using the fact that every element of $\mathrm{B}\left(H_{2}\right)$ can be approximated by an element in $M_{2} \hat{M}_{2}^{\prime}$, we arrive at

$$
\left(\iota \otimes \omega_{1}\right)\left(\Sigma V_{1}^{*} \Sigma\right) \otimes\left(\iota \otimes \omega_{2}\right)\left(W_{2}\right) \in D_{0} .
$$

So, we have proven our claim. In particular, we get that the elements $(\iota \otimes \iota \otimes \omega)\left(Z_{34}^{*} W_{2,24} Z_{34}\left(\Sigma V_{1}^{*} \Sigma\right)_{13}\right)$ are dense in $M_{1} \otimes M_{2}$, because the adjoints of these elements are dense in the range of $\left(R_{1} \otimes R_{2}\right) \eta_{-i / 2}$, which in its turn is dense in $M_{1} \otimes M_{2}$. Because of Proposition 3.5, they are in the image of $\mathrm{m}$ and hence, $\mathrm{m}$ is a *-isomorphism.

Combining with Proposition 3.5, we get that

$$
\mathrm{m}^{-1}\left(R_{1} \otimes R_{2}\right) \eta_{-i / 2}(\iota \otimes \iota \otimes \omega)\left(W_{\mathrm{m}}\right)=(\iota \otimes \iota \otimes \omega)\left(W_{\mathrm{m}}^{*}\right)
$$

and the elements $(\iota \otimes \iota \otimes \omega)\left(W_{\mathrm{m}}\right)$ form a core. Completely analogously, one shows that

$$
\mathrm{m}^{-1}\left(R_{1} \otimes R_{2}\right) \eta_{i / 2}(\iota \otimes \iota \otimes \omega)\left(W_{\mathrm{m}}^{*}\right)=(\iota \otimes \iota \otimes \omega)\left(W_{\mathrm{m}}\right)
$$

and now, the elements $(\iota \otimes \iota \otimes \omega)\left(W_{\mathrm{m}}^{*}\right)$ form a core for $\eta_{i / 2}$. It follows that, as unbounded mappings,

$$
\mathrm{m}^{-1}\left(R_{1} \otimes R_{2}\right) \eta_{-i / 2}=\left(\mathrm{m}^{-1}\left(R_{1} \otimes R_{2}\right) \eta_{i / 2}\right)^{-1}=\eta_{-i / 2}\left(R_{1} \otimes R_{2}\right) \mathrm{m} .
$$

So, still as unbounded mappings, we have

$$
\eta_{-i}=\left(\eta_{-i / 2}\left(R_{1} \otimes R_{2}\right) \mathrm{m}\right)\left(\mathrm{m}^{-1}\left(R_{1} \otimes R_{2}\right) \eta_{-i / 2}\right)=\left(\mathrm{m}^{-1}\left(R_{1} \otimes R_{2}\right) \eta_{-i / 2}\right)\left(\eta_{-i / 2}\left(R_{1} \otimes R_{2}\right) \mathrm{m}\right)
$$

and we conclude that $\left(R_{1} \otimes R_{2}\right) \mathrm{m} \eta_{-i}=\eta_{-i}\left(R_{1} \otimes R_{2}\right) \mathrm{m}$. The right hand side, is equal to $\left(R_{1} \otimes R_{2}\right) \eta_{-i} \mathrm{~m}$ and we get $\mathrm{m} \eta_{-i}=\eta_{-i} \mathrm{~m}$. From Sections 4.3 and 4.4 in [6], it follows that $\mathrm{m}\left(\tau_{-t}^{1} \otimes \tau_{t}^{2}\right)=\left(\tau_{-t}^{1} \otimes \tau_{t}^{2}\right) \mathrm{m}$ for all $t \in \mathbb{R}$. But then, also $\eta_{-i / 2} \mathrm{~m}=\mathrm{m} \eta_{-i / 2}$ and we conclude from Equation (3.1) that $\mathrm{m}^{-1}\left(R_{1} \otimes R_{2}\right)=\left(R_{1} \otimes R_{2}\right) \mathrm{m}$. Hence, we are done. 


\section{More about bicrossed products}

We emphasize again that all notations without indices refer to the bicrossed product quantum group $(M, \Delta)$ associated to our fixed matched pair $\left(M_{1}, \Delta_{1}\right)$ and $\left(M_{2}, \Delta_{2}\right)$, cf. Notation 2.4 .

We give an explicit description of all corepresentations of the bicrossed product $(M, \Delta)$ and deduce from this result some useful information on the modular elements $\delta$ and $\hat{\delta}$ of $(M, \Delta)$ and $(\hat{M}, \hat{\Delta})$.

Proposition 4.1. Suppose that $X$ is a corepresentation of $(M, \Delta)$ on the Hilbert space $K$, i.e. $X \in M \otimes$ $B(K)$ and $(\Delta \otimes \iota)(X)=X_{125} X_{345}$. Then, there exists unique corepresentations $y$ of $\left(M_{2}, \Delta_{2}\right)$ and $z$ of $\left(\hat{M}_{1}, \hat{\Delta}_{1}\right)$ on $K$ such that

$$
X=(\alpha \otimes \iota)(y) z_{13} .
$$

Conversely, if $y$ and $z$ are corepresentations of $\left(M_{2}, \Delta_{2}\right)$ and $\left(\hat{M}_{1}, \hat{\Delta}_{1}\right)$ respectively, on the same Hilbert space $K$, the formula $X=(\alpha \otimes \iota)(y) z_{13}$ defines a corepresentation $X$ of $(M, \Delta)$ if and only if

$$
z_{13}^{*} y_{23} Z_{12}^{*} z_{13} Z_{12} \in M_{1}^{\prime} \otimes \mathrm{B}\left(H_{2} \otimes K\right) .
$$

Proof. Suppose that $X \in M \otimes \mathrm{B}(K)$ is unitary and $(\Delta \otimes \iota)(X)=X_{125} X_{345}$. Because we have a morphism from $(M, \Delta)$ to $\left(\hat{M}_{1}, \hat{\Delta}_{1}\right)$, we can define two actions of $\left(\hat{M}_{1}, \hat{\Delta}_{1}\right)$ on $M$. Explicitly, we have that $\theta: M \rightarrow M \otimes \hat{M}_{1}$ is a right action of $\left(\hat{M}_{1}, \hat{\Delta}_{1}\right)$ while $\mu: M \rightarrow \hat{M}_{1} \otimes M$ is a left action and $\theta, \mu$ are determined by

$$
(\theta \otimes \iota \otimes \iota)(W)=W_{1245}(\iota \otimes \beta)\left(\hat{W}_{1}\right)_{345} \quad \text { and } \quad(\mu \otimes \iota \otimes \iota)(W)=(\iota \otimes \beta)\left(\hat{W}_{1}\right)_{145} W_{2345} .
$$

For a more detailed treatment, see Proposition 3.1 in [15] (but observe that the action $\theta$ there corresponds to our $\sigma \theta$ and is, in that way, a left action of $\left.\left(\hat{M}_{1}, \hat{\Delta}_{1}^{\text {op }}\right)\right)$.

Because $\theta$ is in fact the dual action on the crossed product $M=M_{1 \alpha} \ltimes M_{2}$, we know that $M^{\theta}=\alpha\left(M_{2}\right)$. Further, we observe that $\mu=\sigma\left(R \otimes \hat{R}_{1}\right) \theta R$ and so, $M^{\mu}=\alpha\left(M_{2}\right)$ as well. From Equation (4.1), we conclude that $(\Delta \otimes \iota) \theta=(\iota \otimes \iota \otimes \theta) \Delta$. So, we calculate

$$
(\Delta \otimes \iota \otimes \iota)\left(X_{124}^{*}(\theta \otimes \iota)(X)\right)=X_{346}^{*} X_{126}^{*}(\iota \otimes \iota \otimes \theta \otimes \iota)\left(X_{125} X_{345}\right)=1 \otimes 1 \otimes\left(X_{124}^{*}(\theta \otimes \iota)(X)\right)
$$

and conclude that there exists a unique $z \in \hat{M}_{1} \otimes \mathrm{B}(K)$ such that $(\theta \otimes \iota)(X)=X_{124} z_{34}$. Because applying $\theta \otimes \iota \otimes \iota$ or $\iota \otimes \iota \otimes \hat{\Delta}_{1} \otimes \iota$ to the left hand side of this equation gives the same result, we get $\left(\hat{\Delta}_{1} \otimes \iota\right)(z)=z_{13} z_{23}$.

Define $\gamma_{1}: \hat{M}_{1} \rightarrow M: \gamma_{1}(a)=a \otimes 1$. It is easy to check that $\theta \gamma_{1}=\left(\gamma_{1} \otimes \iota\right) \hat{\Delta}_{1}$. If we apply $\iota \otimes \iota \otimes \theta \gamma_{1} \otimes \iota$ to both sides of the equation $(\theta \otimes \iota)(X)=X_{124} z_{34}$, we conclude that $(\theta \otimes \iota)\left(z_{13}\right)=z_{14} z_{34}$. But then, it follows that $(\theta \otimes \iota)\left(X z_{13}^{*}\right)=\left(X z_{13}^{*}\right)_{124}$ and, because $M^{\theta}=\alpha\left(M_{2}\right)$, we can take $y \in M_{2} \otimes \mathrm{B}(K)$ such that $X z_{13}^{*}=(\alpha \otimes \iota)(y)$. We conclude that $X=(\alpha \otimes \iota)(y) z_{13}$. We want to prove that $\left(\Delta_{2} \otimes \iota\right)(y)=y_{13} y_{23}$.

Because $X$ is a corepresentation and because of the formula for $(\theta \otimes \iota)(X)$, we get $((\theta \otimes \iota \otimes \iota) \Delta \otimes \iota)(X)=$ $X_{126} z_{36} X_{456}$. Using Equation (4.1), we get that $(\theta \otimes \iota \otimes \iota) \Delta=(\iota \otimes \iota \otimes \mu) \Delta$ and so, we conclude that $(\mu \otimes \iota)(X)=z_{14} X_{234}$. Because $X=(\alpha \otimes \iota)(y) z_{13}$ and $\alpha\left(M_{2}\right)=M^{\mu}$, it follows that

$$
(\mu \otimes \iota)\left(z_{13}\right)=(\alpha \otimes \iota)\left(y^{*}\right)_{234} z_{14}(\alpha \otimes \iota)(y)_{234} z_{24}
$$

Next, we get from Equation (4.1) that $\mu(x)=(\iota \otimes \beta)\left(\hat{W}_{1}^{*}\right)(1 \otimes x)(\iota \otimes \beta)\left(\hat{W}_{1}\right)$ for all $x \in M$. In particular, it follows that $\Delta \gamma_{1}=\left(\gamma_{1} \otimes \iota \otimes \iota\right) \mu \gamma_{1}$. Applying this to Equation (4.2), it follows that

$$
(\Delta \otimes \iota)\left(z_{13}\right)=(\alpha \otimes \iota)\left(y^{*}\right)_{345} z_{15}(\alpha \otimes \iota)(y)_{345} z_{35}
$$

Because $X$ is a corepresentation and $X=(\alpha \otimes \iota)(y) z_{13}$, we get

$$
\begin{aligned}
(\alpha \otimes \iota)(y)_{125} z_{15}(\alpha \otimes \iota)(y)_{345} z_{35} & =X_{125} X_{345}=(\Delta \otimes \iota)(X) \\
& =\left((\alpha \otimes \alpha) \Delta_{2} \otimes \iota\right)(y)(\alpha \otimes \iota)\left(y^{*}\right)_{345} z_{15}(\alpha \otimes \iota)(y)_{345} z_{35} .
\end{aligned}
$$

It follows that $\left((\alpha \otimes \alpha) \Delta_{2} \otimes \iota\right)(y)=(\alpha \otimes \iota)(y)_{125}(\alpha \otimes \iota)(y)_{345}$, which finally yields $\left(\Delta_{2} \otimes \iota\right)(y)=y_{13} y_{23}$. 
The uniqueness statement is obvious from the given construction. To prove the converse statement, the formula $z_{13}^{*} y_{23} Z_{12}^{*} z_{13} Z_{12} \in M_{1}^{\prime} \otimes \mathrm{B}\left(H_{2} \otimes K\right)$ is equivalent with

$z_{24}^{*} y_{34} Z_{23}^{*} z_{24} Z_{23} \quad$ commutes with $\hat{W}_{1,12}$,

which is equivalent with

$$
z_{24} \hat{W}_{1,12}^{*} z_{24}^{*} y_{34} Z_{23}^{*} z_{24} Z_{23} \hat{W}_{1,12}=y_{34} Z_{23}^{*} z_{24} Z_{23} .
$$

Using that $z$ is a corepresentation of $\left(\hat{M}_{1}, \hat{\Delta}_{1}\right)$, the last formula is equivalent with

$$
\hat{W}_{1,12}^{*} Z_{23}^{*} z_{24} Z_{23} \hat{W}_{1,12}=y_{34}^{*} z_{14} y_{34} Z_{23}^{*} z_{24} Z_{23} \text {. }
$$

Applying $\operatorname{Ad} Z_{23}$ and using the fact that $\Delta(a \otimes 1)=(\iota \otimes \beta)\left(\hat{W}_{1}^{*}\right)_{134}(1 \otimes 1 \otimes a \otimes 1)(\iota \otimes \beta)\left(\hat{W}_{1}\right)_{134}$ for $a \in \hat{M}_{1}$, the last formula is equivalent with

$$
(\Delta \otimes \iota)\left(z_{13}\right)=(\alpha \otimes \iota)\left(y^{*}\right)_{345} z_{15}(\alpha \otimes \iota)(y)_{345} z_{35} .
$$

Because $y$ is a corepresentation of $\left(M_{2}, \Delta_{2}\right)$ and $\Delta \alpha=(\alpha \otimes \alpha) \Delta_{2}$, it is clear that the final formula is equivalent with $X=(\alpha \otimes \iota)(y) z_{13}$ being a corepresentation of $(M, \Delta)$.

Proposition 4.2. There exist strictly positive, self-adjoint operators $\rho_{1}, \rho_{2}$ affiliated with $M_{1}, M_{2}$ such that

- $\delta^{i t}=\alpha\left(\rho_{2}^{i t}\right)\left(\hat{\delta}_{1}^{i t} \otimes 1\right)$ and both factors commute;

- $\hat{\delta}^{i t}=\beta\left(\rho_{1}^{i t}\right)\left(1 \otimes \hat{\delta}_{2}^{i t}\right)$ and both factors commute;

- $\Delta_{1}\left(\rho_{1}\right)=\rho_{1} \otimes \rho_{1}$ and $\Delta_{2}\left(\rho_{2}\right)=\rho_{2} \otimes \rho_{2}$;

- $\nu=\frac{\nu_{2}}{\nu_{1}}$;

- we have

$$
\begin{aligned}
J \delta^{i t} J & =\hat{J}_{1} \hat{\delta}_{1}^{i t} \hat{J}_{1} \otimes \rho_{2}^{-i t} \delta_{2}^{i t} J_{2} \delta_{2}^{i t} J_{2}, \\
\hat{J} \hat{\delta}^{i t} \hat{J} & =\rho_{1}^{-i t} \delta_{1}^{i t} J_{1} \delta_{1}^{i t} J_{1} \otimes \hat{J}_{2} \hat{\delta}_{2}^{i t} \hat{J}_{2}, \\
{\left[D \varphi_{2} \circ \alpha: D \varphi_{2}\right]_{t} } & =\mathrm{m}\left(\rho_{1}^{-i t / 2} \otimes \rho_{2}^{-i t / 2}\right)\left(\rho_{1}^{-i t / 2} \delta_{1}^{i t} \otimes \rho_{2}^{i t / 2}\right),
\end{aligned}
$$

where we used the Radon-Nikodym derivative introduced in Definition 10.2 of the appendix;

- $\mathrm{m}$ leaves invariant $\rho_{1}^{-i t / 2} \delta_{1}^{i t} \otimes \rho_{2}^{i t / 2} \delta_{2}^{-i t}$.

There exist positive numbers $\lambda_{1,2}>0$ such that $\sigma_{t}^{1}\left(\rho_{1}^{i s}\right)=\lambda_{1}^{\text {ist }} \rho_{1}^{\text {is }}$ and analogously for $\lambda_{2}$ and they satisfy $\frac{\lambda_{2}}{\lambda_{1}}=\frac{\nu_{2}}{\nu_{1}}$.

Proof. Because $\delta^{i t}$ is a one-dimensional corepresentation of $(M, \Delta)$, there exist unique one-dimensional corepresentations (i.e. group-like unitaries) $v_{t} \in \hat{M}_{1}$ and $u_{t} \in M_{2}$ such that $\delta^{i t}=\left(v_{t} \otimes 1\right) \alpha\left(u_{t}\right)$. Because $\alpha$ and $\beta$ are morphisms we know that

$$
\begin{array}{lll}
\operatorname{Ad}\left(\delta^{i t}\right) \circ \alpha=\alpha \circ \operatorname{Ad}\left(\delta_{2}^{i t}\right), & \operatorname{Ad}\left(\hat{\delta}^{i t}\right) \circ \alpha=\alpha \circ \operatorname{Ad}\left(\hat{\delta}_{2}^{i t}\right) & \text { on } M_{2}, \\
\operatorname{Ad}\left(\delta^{i t}\right) \circ \beta=\beta \circ \operatorname{Ad}\left(\hat{\delta}_{1}^{i t}\right), & \operatorname{Ad}\left(\hat{\delta}^{i t}\right) \circ \beta=\beta \circ \operatorname{Ad}\left(\delta_{1}^{i t}\right) & \text { on } M_{1} .
\end{array}
$$

Then,

$$
\begin{aligned}
\Delta^{\mathrm{op}}\left(v_{t} \otimes 1\right) & =(\beta \otimes \iota)\left(W_{1}\right)\left(v_{t} \otimes 1 \otimes 1\right)(\beta \otimes \iota)\left(W_{1}^{*}\right) \otimes 1 \\
& =\left((\beta \otimes \iota)\left(W_{1}\right)\left(\delta^{i t} \otimes 1\right)(\beta \otimes \iota)\left(W_{1}^{*}\right) \otimes 1\right)\left(\alpha\left(u_{t}^{*}\right) \otimes 1 \otimes 1\right) \\
& =\left((\beta \otimes \iota)\left(W_{1}\right)\left(\beta \circ \operatorname{Ad}\left(\hat{\delta}_{1}^{i t}\right) \otimes \iota\right)\left(W_{1}^{*}\right)\left(v_{t} \otimes 1 \otimes 1\right)\right) \otimes 1=v_{t} \otimes 1 \otimes \hat{\delta}_{1}^{i t} \otimes 1 .
\end{aligned}
$$

Hence, $\Delta^{\mathrm{op}}\left(\delta^{i t}\right)=\Delta^{\mathrm{op}}\left(\left(v_{t} \otimes 1\right) \alpha\left(u_{t}\right)\right)=\left(v_{t} \otimes 1\right) \alpha\left(u_{t}\right) \otimes\left(\hat{\delta}_{1}^{i t} \otimes 1\right) \alpha\left(u_{t}\right)$. Because $\delta^{i t}$ is group-like, we conclude that $v_{t}=\hat{\delta}_{1}^{i t}$. 
At the end of this proof, we will argue that in a l.c. quantum group, the modular element commutes with any group-like unitary. So, $\delta^{i t}$ commutes with $\alpha\left(u_{s}\right)$ for all $s, t \in \mathbb{R}$. As $\delta^{i t}$ commutes with $\delta^{i s}$, we get that $\delta^{i t}$ commutes with $\hat{\delta}_{1}^{i s} \otimes 1$ for all $s, t \in \mathbb{R}$. This means that $\left(\left(\hat{\delta}_{1}^{-i t} \otimes 1\right) \delta^{i t}\right)_{t}$ is a one-parameter group of unitaries. Then, there exists a unique strictly positive, self-adjoint operator $\rho_{2}$ affiliated with $M_{2}$ such that $\delta^{i t}=\left(\hat{\delta}_{1}^{i t} \otimes 1\right) \alpha\left(\rho_{2}^{i t}\right)$. By symmetry, we find $\rho_{1}$ and we have proven the first three statements of the proposition.

Let $a \in \mathcal{N}_{\hat{\varphi}_{1}}$ and $x \in \mathcal{N}_{\varphi_{2}}$. Then, we have

$$
(a \otimes 1) \alpha(x) \delta^{i t}=(a \otimes 1) \delta^{i t} \alpha\left(\delta_{2}^{-i t} x \delta_{2}^{i t}\right)=\left(a \hat{\delta}_{1}^{i t} \otimes 1\right) \alpha\left(\rho_{2}^{i t} \delta_{2}^{-i t} x \delta_{2}^{i t}\right) .
$$

We conclude that

$$
\Lambda\left((a \otimes 1) \alpha(x) \delta^{i t}\right)=\left(\frac{\nu_{2}}{\nu_{1}}\right)^{-t / 2}\left(\hat{J}_{1} \hat{\delta}_{1}^{-i t} \hat{J}_{1} \otimes \rho_{2}^{i t} \delta_{2}^{-i t} J_{2} \delta_{2}^{-i t} J_{2}\right) \Lambda((a \otimes 1) \alpha(x)) .
$$

On the other hand, we know that

$$
\Lambda\left((a \otimes 1) \alpha(x) \delta^{i t}\right)=\nu^{-t / 2} J \delta^{-i t} J \Lambda((a \otimes 1) \alpha(x)) .
$$

So, $\nu=\frac{\nu_{2}}{\nu_{1}}$ and $J \delta^{i t} J=\hat{J}_{1} \hat{\delta}_{1}^{i t} \hat{J}_{1} \otimes \rho_{2}^{-i t} \delta_{2}^{i t} J_{2} \delta_{2}^{i t} J_{2}$. We find the formula for $\hat{J} \hat{\delta}^{i t} \hat{J}$ in an analogous way.

From [16], Proposition 2.4, we know that

$$
\nabla^{i t}=\hat{\delta}^{-i t / 2} \delta^{-i t / 2} J \delta^{-i t / 2} J \hat{J} \hat{\delta}^{i t / 2} \hat{J}
$$

We can take positive numbers $\lambda_{1,2}>0$ such that $\sigma_{t}^{1}\left(\rho_{1}^{i s}\right)=\lambda_{1}^{i s t} \rho_{1}^{i s}$ and analogously for $\lambda_{2}$ (see the last paragraph of this proof). Because in any l.c. quantum group, $\operatorname{Ad} \hat{\delta}^{i t}=\tau_{-t} \operatorname{Ad}\left(\delta^{-i t}\right) \sigma_{-t}$ on $M$, we easily find the required formula for $\left[D \varphi_{2} \circ \alpha: D \varphi_{2}\right]_{t}:=\nabla^{i t}\left(\hat{\nabla}_{1}^{-i t} \otimes \nabla_{2}^{-i t}\right)$. We also get that $\hat{\delta}^{-i s} \alpha\left(\rho_{2}^{i t}\right) \hat{\delta}^{i s}=\lambda_{2}^{i s t} \alpha\left(\rho_{2}^{i t}\right)$ and $\delta^{i t} \beta\left(\rho_{1}^{i s}\right) \delta^{-i t}=\lambda_{1}^{-i s t} \beta\left(\rho_{1}^{i s}\right)$. A short calculation yields that $\delta^{i t} \hat{\delta}^{i s}=\left(\frac{\lambda_{2}}{\lambda_{1}}\right)^{i s t} \hat{\delta}^{i s} \delta^{i t}$. But, from the general theory, we know that $\delta^{i t} \hat{\delta}^{i s}=\nu^{i s t} \hat{\delta}^{i s} \delta^{i t}$, giving us the equality $\frac{\lambda_{2}}{\lambda_{1}}=\frac{\nu_{2}}{\nu_{1}}$.

Consider $\Delta_{2}^{\mathrm{op}}$ as an action of $\left(M_{2}, \Delta_{2}^{\mathrm{op}}\right)$ on $M_{2}$. Then, $\left[D \varphi_{2} \circ \Delta_{2}^{\mathrm{op}}: D \varphi_{2}\right]_{t}=\delta_{2}^{-i t} \otimes 1$. Because $\left(\iota \otimes \Delta_{2}^{\mathrm{op}}\right) \alpha=$ $(\mathrm{m} \sigma \otimes \iota)(\iota \otimes \alpha) \Delta_{2}^{\mathrm{op}}$ and because, by Lemma 3.6, $\mathrm{m}\left(\tau_{-t}^{1} \otimes \tau_{t}^{2}\right)=\left(\tau_{-t}^{1} \otimes \tau_{t}^{2}\right) \mathrm{m}$, we can apply Lemma 10.4 of our Appendix to conclude that

$$
\left(\iota \otimes \Delta_{2}^{\mathrm{op}}\right)\left(\left[D \varphi_{2} \circ \alpha: D \varphi_{2}\right]_{t}\right)=(\mathrm{m} \sigma \otimes \iota)\left(\delta_{2}^{-i t} \otimes\left[D \varphi_{2} \circ \alpha: D \varphi_{2}\right]_{t}\right)\left(1 \otimes \delta_{2}^{i t} \otimes 1\right) .
$$

Filling in the already obtained formula for $\left[D \varphi_{2} \circ \alpha: D \varphi_{2}\right]_{t}$ and carefully rewriting, we arrive at the final statement of the proposition.

As we promised above, we consider now a group-like unitary $u \in M$ for some l.c. quantum group $(M, \Delta)$. By the uniqueness of the Haar measure, we find $\lambda>0$ such that $\varphi\left(u x u^{*}\right)=\lambda \varphi(x)$ for all $x \in M^{+}$. It follows that $\sigma_{t}(u)=\lambda^{i t} u$ for all $t \in \mathbb{R}$. Because a group-like unitary is a one-dimensional corepresentation, we have $u \in D(S)$ and $S(u)=u^{*}$. But, also $u^{*}$ is a group-like element and so $S^{2}(u)=u$. It follows that $\tau_{t}(u)=u$ for all $t \in \mathbb{R}$ and $R(u)=u^{*}$. Then $\sigma_{t}^{\prime}(u)=R \sigma_{-t} R(u)=\lambda^{i t} u$, which gives $\sigma_{t}^{\prime} \sigma_{-t}(u)=u$ and so, $u$ commutes with $\delta^{i t}$ for all $t \in \mathbb{R}$.

\section{Double crossed products as locally compact quantum groups}

In this section, we compute the invariant weights on $\left(M_{\mathrm{m}}, \Delta_{\mathrm{m}}\right)$ and obtain that $\left(M_{\mathrm{m}}, \Delta_{\mathrm{m}}\right)$ is a l.c. quantum group. We compute all the operators associated with $\left(M_{\mathrm{m}}, \Delta_{\mathrm{m}}\right)$.

Notation 5.1. We use $\rho_{1}, \rho_{2}$ to denote the unbounded operators introduced in Proposition 4.2. We define strictly positive, self-adjoint operators $k_{1}, k_{2}$ such that

$$
k_{1}^{i t}=\rho_{1}^{i t} \delta_{1}^{-i t} \quad \text { and } \quad k_{2}^{i t}=\rho_{2}^{i t} \delta_{2}^{-i t} .
$$


In order to obtain invariant weights for $\left(M_{\mathrm{m}}, \Delta_{\mathrm{m}}\right)$, we need the following relative invariance result of the weight $\psi_{2}$ under the action $\alpha$.

Proposition 5.2. For all $x \in \mathcal{N}_{\psi_{2}}, \xi \in H_{1}$ and $\eta \in D\left(k_{1}^{1 / 2}\right)$, we have $\left(\omega_{\xi, \eta} \otimes \iota\right)\left(U_{\beta}^{*} \alpha(x)\right) \in \mathcal{N}_{\psi_{2}}$ and

$$
\Gamma_{2}\left(\left(\omega_{\xi, \eta} \otimes \iota\right)\left(U_{\beta}^{*} \alpha(x)\right)\right)=\left(\omega_{\xi, k_{1}^{1 / 2} \eta} \otimes \iota\right)\left(U_{\beta}^{*} U_{\alpha}\right) \Gamma_{2}(x) .
$$

On the other hand, for $x \in \mathcal{N}_{\psi_{1}}, \xi \in H_{2}$ and $\eta \in D\left(k_{2}^{1 / 2}\right)$, we have $\left(\iota \otimes \omega_{\xi, \eta}\right)\left(U_{\alpha}^{*} \beta(x)\right) \in \mathcal{N}_{\psi_{1}}$ and

$$
\Gamma_{1}\left(\left(\iota \otimes \omega_{\xi, \eta}\right)\left(U_{\alpha}^{*} \beta(x)\right)\right)=\left(\iota \otimes \omega_{\xi, k_{2}^{1 / 2} \eta}\right)\left(U_{\alpha}^{*} U_{\beta}\right) \Gamma_{1}(x) .
$$

Proof. Consider the dual weight $\tilde{\psi}_{2}$ on $M$ with a canonical GNS-map $\tilde{\Gamma}$ determined by

$$
\tilde{\Gamma}((a \otimes 1) \alpha(x))=\hat{\Lambda}_{1}(a) \otimes \Gamma_{2}(x) \quad \text { for all } \quad a \in \mathcal{N}_{\hat{\varphi}_{1}}, x \in \mathcal{N}_{\psi_{2}} .
$$

We denote by $\tilde{\nabla}$ the modular operator of $\tilde{\psi}_{2}$ in this GNS-construction. Observe that $\psi_{2}=\left(\varphi_{2}\right)_{\delta_{2}}$ and hence, $\tilde{\psi}_{2}=\varphi_{\alpha\left(\delta_{2}\right)}$. It then follows from [14], Proposition 2.5 that

$$
\begin{aligned}
\tilde{\nabla}^{i t} & =J \alpha\left(\delta_{2}^{i t}\right) J \alpha\left(\delta_{2}^{i t}\right) \nabla^{i t}, \\
\tilde{T} & =\nu_{2}^{i / 4} J \tilde{\nabla}^{1 / 2},
\end{aligned}
$$

where $\tilde{T}$ is determined by $\tilde{T} \tilde{\Gamma}(z)=\tilde{\Gamma}\left(z^{*}\right)$. From Proposition 4.2 , it follows that

$$
\begin{aligned}
\tilde{\nabla}^{i t} & =\alpha\left(\delta_{2}^{i t}\right)\left(1 \otimes J_{2} \delta_{2}^{i t} J_{2}\right) \nabla^{i t}=\alpha\left(\delta_{2}^{i t}\right)\left(1 \otimes J_{2} \delta_{2}^{i t} J_{2}\right)\left[D \varphi_{2} \circ \alpha: D \varphi_{2}\right]_{t}\left(\hat{\nabla}_{1}^{i t} \otimes \nabla_{2}^{i t}\right) \\
& =\mathrm{m}\left(\rho_{1}^{-i t / 2} \otimes \rho_{2}^{-i t / 2} \delta_{2}^{i t}\right)\left(\rho_{1}^{-i t / 2} \delta_{1}^{i t} \otimes \rho_{2}^{i t / 2} \delta_{2}^{-i t}\right)\left(\hat{\nabla}_{1}^{i t} \otimes \nabla_{2}^{i t}\right)=\beta\left(k_{1}^{-i t}\right)\left(\hat{\nabla}_{1}^{i t} \otimes \nabla_{2}^{i t}\right),
\end{aligned}
$$

where $\nabla_{2}$ denotes the modular operator of $\psi_{2}$.

We know that the elements $\alpha\left(x^{*}\right)\left(\mu \otimes \Gamma_{2}(y)\right), x, y \in \mathcal{N}_{\psi_{2}}, \mu \in D\left(\hat{\nabla}_{1}^{1 / 2}\right)$ span a core for $\tilde{T}$ and

$$
\tilde{T} \alpha\left(x^{*}\right)\left(\mu \otimes \Gamma_{2}(y)\right)=\alpha\left(y^{*}\right)\left(\hat{J}_{1} \hat{\nabla}_{1}^{1 / 2} \mu \otimes \Gamma_{2}(x)\right) .
$$

It follows that

$$
\tilde{\nabla}^{1 / 2} \alpha\left(x^{*}\right)\left(\mu \otimes \Gamma_{2}(y)\right)=\nu_{2}^{i / 4}\left(1 \otimes J_{2} y^{*} J_{2}\right) U_{\alpha}\left(\hat{\nabla}_{1}^{1 / 2} \mu \otimes J_{2} \Gamma_{2}(x)\right) .
$$

Because the product of $\beta\left(k_{1}^{-i t}\right)$ and $\hat{\nabla}_{1}^{i t} \otimes \nabla_{2}^{i t}$ yields the one-parameter group $\tilde{\nabla}^{i t}$, we get that $\beta\left(k_{1}\right)$ and $\hat{\nabla}_{1} \otimes \nabla_{2}$ commute strongly. If we assume now that $x$ is analytic w.r.t. the modular group $\left(\sigma_{t}^{2 \prime}\right)$ of $\psi_{2}$ and $y \in \mathcal{N}_{\psi_{2}} \cap \mathcal{N}_{\psi_{2}}^{*}$, we conclude that the left hand side of the previous equality is equal to

$$
\nu_{2}^{i / 4} \beta\left(k_{1}^{-1 / 2}\right) \alpha\left(\sigma_{-i / 2}^{2 \prime}\left(x^{*}\right)\right)\left(\hat{\nabla}_{1}^{1 / 2} \mu \otimes J_{2} \Gamma_{2}\left(y^{*}\right)\right) .
$$

By a typical density argument, we may conclude that for $x \in \mathcal{N}_{\psi_{2}}$, analytic w.r.t. $\left(\sigma_{t}^{2 \prime}\right), y \in \mathcal{N}_{\psi_{2}}$ and $\xi \in H_{1}$,

$$
\beta\left(k_{1}^{-1 / 2}\right) \alpha\left(\sigma_{-i / 2}^{2 \prime}\left(x^{*}\right)\right)\left(\xi \otimes J_{2} \Gamma_{2}(y)\right)=\left(1 \otimes J_{2} y J_{2}\right) U_{\alpha}\left(\xi \otimes J_{2} \Gamma_{2}(x)\right) .
$$

Applying $U_{\beta}^{*} \in \mathrm{B}\left(H_{1}\right) \otimes M_{2}$, it follows that for $x, y, \xi$ as above and $\eta \in D\left(k_{1}^{1 / 2}\right)$,

$$
\left(\omega_{\xi, \eta} \otimes \iota\right)\left(U_{\beta}^{*} \alpha\left(\sigma_{-i / 2}^{2 \prime}\left(x^{*}\right)\right)\right) J_{2} \Gamma_{2}(y)=J_{2} y J_{2}\left(\omega_{\xi, k_{1}^{1 / 2} \eta} \otimes \iota\right)\left(U_{\beta}^{*} U_{\alpha}\right) J_{2} \Gamma_{2}(x) .
$$

Using $J_{2} \Gamma_{2}(z)=\Gamma_{2}\left(\sigma_{-i / 2}^{2 \prime}\left(z^{*}\right)\right)$, we get, for $x, y \in \mathcal{N}_{\psi_{2}}, y$ analytic w.r.t. $\left(\sigma_{t}^{2 \prime}\right), \xi \in H_{1}$ and $\eta \in D\left(k_{1}^{1 / 2}\right)$,

$$
\Gamma_{2}\left(\left(\omega_{\xi, \eta} \otimes \iota\right)\left(U_{\beta}^{*} \alpha(x)\right) y\right)=J_{2} \sigma_{i / 2}^{2 \prime}(y)^{*} J_{2}\left(\omega_{\xi, k^{1 / 2} \eta} \otimes \iota\right)\left(U_{\beta}^{*} U_{\alpha}\right) \Gamma_{2}(x) .
$$

From this formula, our first result follows. The second result is analogous. 
We now prove our main result: the pair $\left(M_{\mathrm{m}}, \Delta_{\mathrm{m}}\right)$ is a l.c. quantum group and we calculate all its structural ingredients. Observe that in the case of ordinary l.c. group matchings, the same kind of formulas were obtained in [15], Propositions 4.14 and 4.15 .

Theorem 5.3. The pair $\left(M_{\mathrm{m}}, \Delta_{\mathrm{m}}\right)$ is a l.c. quantum group. The weight $\varphi_{\mathrm{m}}:=\psi_{1} \otimes\left(\varphi_{2}\right)_{k_{2}}$ is left invariant and

$$
W_{\mathrm{m}}=\left(\Sigma V_{1}^{*} \Sigma\right)_{13} Z_{34}^{*} W_{2,24} Z_{34}=\left(\Sigma V_{1}^{*} \Sigma\right)_{13} U_{\beta, 32}^{*}(\alpha \otimes \iota)\left(W_{2}\right)_{324}
$$

is the left regular representation when we use the canonical GNS-construction for $\varphi_{\mathrm{m}}$.

The canonical right invariant weight $\psi_{\mathrm{m}}$ is given by $\left(\varphi_{1}\right)_{k_{1}} \otimes \psi_{2}$ and the right regular representation by

$$
V_{\mathrm{m}}=Z_{12}^{*} \hat{W}_{1,13} Z_{12} V_{2,24}=(\iota \otimes \beta)\left(\hat{W}_{1}\right)_{132} U_{\alpha, 32} V_{2,24} .
$$

Further, we have

$$
\begin{array}{rlrl}
R_{\mathrm{m}} & =\mathrm{m}^{-1}\left(R_{1} \otimes R_{2}\right)=\left(R_{1} \otimes R_{2}\right) \mathrm{m}, & \nu_{\mathrm{m}} & =\frac{\nu_{2}}{\nu_{1}}, \\
\tau_{t}^{\mathrm{m}} & =\tau_{-t}^{1} \otimes \tau_{t}^{2}, & P_{\mathrm{m}} & =P_{1}^{-1} \otimes P_{2}, \\
J_{\mathrm{m}} & =\lambda_{1}^{i / 4}\left(J_{1} \otimes J_{2}\right), & \hat{\delta}_{\mathrm{m}}^{i t} & =\left(\hat{J}_{1} \hat{\delta}_{1}^{-i t} \hat{J}_{1} \otimes 1\right) Z^{*}\left(1 \otimes \hat{\delta}_{2}^{i t}\right) Z, \\
\hat{J}_{\mathrm{m}} & =\lambda_{1}^{i / 4}\left(\hat{J}_{1} \otimes \hat{J}_{2}\right) J \hat{J}\left(\hat{J}_{1} J_{1} \otimes \hat{J}_{2} J_{2}\right), & \hat{J}_{\mathrm{m}} \hat{\delta}_{\mathrm{m}}^{i t} \hat{J}_{\mathrm{m}}=\left(1 \otimes \hat{J}_{2} \hat{\delta}_{2}^{i t} \hat{J}_{2}\right) Z^{*}\left(\hat{\delta}_{1}^{-i t} \otimes 1\right) Z, \\
\delta_{\mathrm{m}}^{i t} & =\rho_{1}^{i t} \delta_{1}^{-2 i t} \otimes \rho_{2}^{-i t} \delta_{2}^{2 i t}, &
\end{array}
$$

where $\lambda_{1}>0$ is defined in Proposition 4.2. The dual of $\left(M_{\mathrm{m}}, \Delta_{\mathrm{m}}\right)$ is given by

$$
\hat{M}_{\mathrm{m}}=\left(\hat{M}_{1}^{\prime} \otimes 1 \cup Z^{*}\left(1 \otimes \hat{M}_{2}\right) Z\right)^{\prime \prime} \quad \text { and } \quad \hat{M}_{\mathrm{m}}^{\prime}=\left(Z^{*}\left(\hat{M}_{1} \otimes 1\right) Z \cup 1 \otimes \hat{M}_{2}^{\prime}\right)^{\prime \prime},
$$

and in this picture, $\left(\hat{M}_{1}^{\prime}, \hat{\Delta}_{1}^{\prime}\right)$ and $\left(\hat{M}_{2}, \hat{\Delta}_{2}\right)$ are closed quantum subgroups of $\left(\hat{M}_{\mathrm{m}}, \hat{\Delta}_{\mathrm{m}}\right)$.

Finally, we have the following commutation relations

$$
\begin{aligned}
J \hat{J}\left(J_{1} \otimes J_{2}\right) & =\left(\frac{\nu_{1}}{\nu_{2}}\right)^{i / 4}\left(J_{1} \otimes J_{2}\right) J \hat{J}, \\
(\iota \otimes \mathrm{m})\left(U_{\beta, 13} V_{1,12}\right) & =V_{1,12} U_{\beta, 13}, \\
(\mathrm{~m} \otimes \iota)\left(U_{\alpha, 13}\left(\Sigma V_{2} \Sigma\right)_{23}\right) & =\left(\Sigma V_{2} \Sigma\right)_{23} U_{\alpha, 13} .
\end{aligned}
$$

Recall that the notations $Z$ and $W_{\mathrm{m}}$ are introduced in Notation 3.2 and Proposition 3.5.

Proof. Define $\varphi_{\mathrm{m}}:=\psi_{1} \otimes\left(\varphi_{2}\right)_{k_{2}}$ and denote by $\Lambda_{\mathrm{m}}$ its canonical GNS-map. Let $x \in \mathcal{N}_{\psi_{1}}, y, z \in \mathcal{N}_{\left(\varphi_{2}\right)_{k_{2}}}$ where $z$ is analytic w.r.t. the modular group of $\left(\varphi_{2}\right)_{k_{2}}$. Take $\xi, \eta \in H_{1}$ and $\mu, \rho \in H_{2}$. Then,

$$
\left(\omega_{\xi \otimes \mu, \eta \otimes \rho} \otimes \iota \otimes \iota\right) \Delta_{\mathrm{m}}(x \otimes y)(1 \otimes z)=\left(\iota \otimes \omega_{\mu, \rho} \otimes \iota\right)\left(\left(U_{\alpha} \otimes 1\right)\left(1 \otimes \Delta_{2}(y)\right)\left(U_{\alpha}^{*} \beta\left(\left(\iota \otimes \omega_{\xi, \eta}\right) \Delta_{1}(x)\right) \otimes z\right)\right) .
$$

Let $\chi_{[1 / n, n]}$ be the characteristic function of the interval $[1 / n, n]$ and define $P_{n}=\chi_{[1 / n, n]}\left(k_{2}\right)$. Let $\left(e_{i}\right)$ be an orthonormal basis for $H_{2}$. Consider the following element in $M_{1} \otimes M_{2}$ :

$$
\begin{aligned}
r & :=\left(\iota \otimes \omega_{\mu, \rho} \otimes \iota\right)\left(\left(U_{\alpha} \otimes 1\right)\left(1 \otimes \Delta_{2}(y)\right)\left(1 \otimes P_{n} \otimes 1\right)\left(U_{\alpha}^{*} \beta\left(\left(\iota \otimes \omega_{\xi, \eta}\right) \Delta_{1}(x)\right) \otimes z\right)\right) \\
& =\sum_{i}\left(\iota \otimes \omega_{e_{i}, \rho} \otimes \iota\right)\left(\left(U_{\alpha} \otimes 1\right)\left(1 \otimes \Delta_{2}(y)\right)\right)\left(\left(\iota \otimes \omega_{\mu, P_{n} e_{i}}\right)\left(U_{\alpha}^{*} \beta\left(\left(\iota \otimes \omega_{\xi, \eta}\right) \Delta_{1}(x)\right)\right) \otimes z\right) .
\end{aligned}
$$

Denote every individual term of this sum by $r_{i}$. Because of Proposition 5.2, every $r_{i}$ belongs to $\mathcal{N}_{\varphi_{\mathrm{m}}}$ and

$$
\Lambda_{\mathrm{m}}\left(r_{i}\right)=\left(\iota \otimes \omega_{e_{i}, \rho} \otimes \iota\right)\left(\left(U_{\alpha} \otimes 1\right)\left(1 \otimes \Delta_{2}(y)\right)\right)\left(\left(\iota \otimes \omega_{\mu, k_{2}^{1 / 2} P_{n} e_{i}}\right)\left(U_{\alpha}^{*} U_{\beta}\right)\left(\iota \otimes \omega_{\xi, \eta}\right)\left(V_{1}\right) \Gamma_{1}(x) \otimes \Lambda_{\left(\varphi_{2}\right)_{k_{2}}}(z)\right) .
$$

This expression is summable over $i$ and we conclude that $r \in \mathcal{N}_{\varphi_{\mathrm{m}}}$ and

$$
\Lambda_{\mathrm{m}}(r)=\left(\iota \otimes \omega_{\mu, \rho} \otimes \iota\right)\left((\alpha \otimes \iota)\left(\Delta_{2}(y)\left(\left(k_{2}^{1 / 2} P_{n}\right) \otimes 1\right)\right)\left(U_{\beta} \otimes 1\right)\right)\left(\left(\iota \otimes \omega_{\xi, \eta}\right)\left(V_{1}\right) \Gamma_{1}(x) \otimes \Lambda_{\left(\varphi_{2}\right)_{k_{2}}}(z)\right) .
$$


Write $v:=\left(\iota \otimes \omega_{\xi, \eta}\right)\left(V_{1}\right) \Gamma_{1}(x)$ and take $w \in H_{1}$. Then, denoting by $\left(\tilde{\sigma}_{t}\right)$ the modular group of $\left(\varphi_{2}\right)_{k_{2}}$,

$$
\begin{aligned}
\left(\theta_{w}^{*} \otimes \iota\right) \Lambda_{\mathrm{m}}(r) & =\Lambda_{\left(\varphi_{2}\right)_{k_{2}}}\left(\left(\omega_{U_{\beta}(v \otimes \mu), w \otimes \rho} \circ \alpha \otimes \iota\right)\left(\Delta_{2}(y)\left(\left(k_{2}^{1 / 2} P_{n}\right) \otimes 1\right)\right) z\right) \\
& =J_{2} \tilde{\sigma}_{i / 2}(z)^{*} J_{2}\left(\omega_{\alpha\left(P_{n}\right) U_{\beta}(v \otimes \mu), w \otimes \rho} \circ \alpha \otimes \iota\right)\left(W_{2}^{*}\right) \Lambda_{\left(\varphi_{2}\right)_{k_{2}}}(y),
\end{aligned}
$$

where we used that $k_{2}$ is a group-like. Hence,

$$
\Lambda_{\mathrm{m}}(r)=\left(1 \otimes J_{2} \tilde{\sigma}_{i / 2}(z)^{*} J_{2}\right)\left(\iota \otimes \omega_{\mu, \rho} \otimes \iota\right)\left((\alpha \otimes \iota)\left(W_{2}^{*}\right)\left(\alpha\left(P_{n}\right) U_{\beta} \otimes 1\right)\right)\left(\left(\iota \otimes \omega_{\xi, \eta}\right)\left(V_{1}\right) \Gamma_{1}(x) \otimes \Lambda_{\left(\varphi_{2}\right)_{k_{2}}}(y)\right) .
$$

Taking limits over $n \rightarrow \infty$ and $z \rightarrow 1$, we get that

$$
\left(\omega_{\xi \otimes \mu, \eta \otimes \rho} \otimes \iota \otimes \iota\right) \Delta_{\mathrm{m}}(x \otimes y) \in \mathcal{N}_{\varphi_{\mathrm{m}}}
$$

and

$$
\Lambda_{\mathrm{m}}\left(\left(\omega_{\xi \otimes \mu, \eta \otimes \rho} \otimes \iota \otimes \iota\right) \Delta_{\mathrm{m}}(x \otimes y)\right)=\left(\omega_{\xi \otimes \mu, \eta \otimes \rho} \otimes \iota \otimes \iota\right)\left((\alpha \otimes \iota)\left(W_{2}^{*}\right)_{324} U_{\beta, 32}\left(\Sigma V_{1} \Sigma\right)_{13}\right) \Lambda_{\mathrm{m}}(x \otimes y) .
$$

It follows that $\varphi_{\mathrm{m}}$ is left invariant and that its associated left regular representation is given by

$$
\mathcal{W}_{\mathrm{m}}:=\left(\Sigma V_{1}^{*} \Sigma\right)_{13} U_{\beta, 32}^{*}(\alpha \otimes \iota)\left(W_{2}\right)_{324}
$$

Define $R_{\mathrm{m}}:=\left(R_{1} \otimes R_{2}\right) \mathrm{m}$. From Lemma 3.6, we know that $R_{\mathrm{m}}=\mathrm{m}^{-1}\left(R_{1} \otimes R_{2}\right)$ as well and then, it is easy to verify that $\Delta_{\mathrm{m}} R_{\mathrm{m}}=\left(R_{\mathrm{m}} \otimes R_{\mathrm{m}}\right) \Delta_{\mathrm{m}}^{\mathrm{op}}$.

So, $\varphi_{\mathrm{m}} R_{\mathrm{m}}$ is a right invariant weight and $\left(M_{\mathrm{m}}, R_{\mathrm{m}}\right)$ is a l.c. quantum group. It follows that $\mathcal{W}_{\mathrm{m}}$ is a multiplicative unitary and we want to prove that $\mathcal{W}_{\mathrm{m}}=W_{\mathrm{m}}$.

An analogous computation as above shows that $\left(\varphi_{1}\right)_{k_{1}} \otimes \psi_{2}$ is a right invariant weight with associated right regular representation

$$
V_{\mathrm{m}}:=(\iota \otimes \beta)\left(\hat{W}_{1}\right)_{132} U_{\alpha, 32} V_{2,24} .
$$

Observe that

$$
\begin{aligned}
\left(\Delta_{\mathrm{m}} \otimes \iota \otimes \iota\right)\left(\mathcal{W}_{\mathrm{m}}\right) & \left.=\sigma_{23} \mathrm{~m}_{23}\left(\left(\Sigma V_{1}^{*} \Sigma\right)_{15}\left(\Sigma V_{1}^{*} \Sigma\right)_{25} U_{\beta, 53}^{*} U_{\beta, 54}^{*}\left(\iota \otimes \Delta_{2}\right) \alpha \otimes \iota\right)\left(W_{2}\right)_{5346}\right) \\
& =\sigma_{23} \mathrm{~m}_{23}\left(\left(\Sigma V_{1}^{*} \Sigma\right)_{15}\left(\Sigma V_{1}^{*} \Sigma\right)_{25} U_{\beta, 53}^{*} U_{\beta, 54}^{*}\left(\mathrm{~m}_{13}(\alpha \otimes \iota \otimes \iota)\left(W_{2,13} W_{2,23}\right)\right)_{5346}\right) \\
& \left.=\left(\Sigma V_{1}^{*} \Sigma\right)_{15}\left((\iota \otimes \mathrm{m})\left(V_{1,12}^{*} U_{\beta, 13}^{*}\right)\right)_{532}(\iota \otimes \alpha) \alpha \otimes \iota\right)\left(W_{2}\right)_{5326} U_{\beta, 54}^{*}(\alpha \otimes \iota)\left(W_{2}\right)_{546} .
\end{aligned}
$$

On the other hand, we know that

$$
\left(\Delta_{\mathrm{m}} \otimes \iota \otimes \iota\right)\left(\mathcal{W}_{\mathrm{m}}\right)=\left(\mathcal{W}_{\mathrm{m}}\right)_{1256}\left(\mathcal{W}_{\mathrm{m}}\right)_{3456}=\left(\Sigma V_{1}^{*} \Sigma\right)_{15} U_{\beta, 52}^{*}(\alpha \otimes \iota)\left(W_{2}\right)_{526}\left(\Sigma V_{1}^{*} \Sigma\right)_{35} U_{\beta, 54}^{*}(\alpha \otimes \iota)\left(W_{2}\right)_{546}
$$

We conclude that

$$
(\iota \otimes \mathrm{m})\left(U_{\beta, 13} V_{1,12}\right)=V_{1,12} U_{\beta, 13} .
$$

Making an analogous computation with $V_{\mathrm{m}}$, we arrive at the commutation relations (5.1) and (5.2).

We now claim that

$$
Z_{23}^{*} W_{2,13}^{*} Z_{23}=(\alpha \otimes \iota)\left(W_{2}^{*}\right)_{213} U_{\beta, 21},
$$

yielding the equality $\mathcal{W}_{\mathrm{m}}=W_{\mathrm{m}}$. Analogously, one proves the second formula for the right regular representation: $V_{\mathrm{m}}=Z_{12}^{*} \hat{W}_{1,13} Z_{12} V_{2,24}$. To prove the claim, we make the following calculation:

$$
\begin{aligned}
Z_{23}^{*} W_{2,13}^{*} Z_{23} & =\left(\hat{J}_{2} \otimes J_{1} \otimes \hat{J}_{2}\right) U_{\alpha, 23}^{*} U_{\beta, 23}\left(\iota \otimes \Delta_{2}^{\mathrm{op}}\right)\left(U_{\beta}^{*}\right)_{213}\left(\Sigma V_{2} \Sigma\right)_{13} U_{\alpha, 23}\left(\hat{J}_{2} \otimes J_{1} \otimes \hat{J}_{2}\right) \\
& =\left(\hat{J}_{2} \otimes J_{1} \otimes \hat{J}_{2}\right) U_{\alpha, 23}^{*} U_{\beta, 21}^{*}\left(\Sigma V_{2} \Sigma\right)_{13} U_{\alpha, 23}\left(\hat{J}_{2} \otimes J_{1} \otimes \hat{J}_{2}\right) .
\end{aligned}
$$

Observe that

$$
Z^{*}=\nu_{1}^{-i / 4} \tilde{U}_{\alpha}^{*} U_{\beta}^{*} \quad \text { where } \quad \tilde{U}_{\alpha}=\left(J_{1} \otimes \hat{J}_{2}\right) U_{\alpha}\left(J_{1} \otimes \hat{J}_{2}\right) \in M_{1}^{\prime} \otimes \mathrm{B}\left(H_{2}\right)
$$


We combine Lemma 3.3 and Equation (5.2) with the calculation above, to get

$$
\begin{aligned}
Z_{23}^{*} W_{2,13}^{*} Z_{23} & =\nu_{1}^{-i / 4}\left(\hat{J}_{2} \otimes J_{1} \otimes \hat{J}_{2}\right) U_{\alpha, 23}^{*} \tilde{U}_{\alpha, 21} Z_{21}^{*}\left(\Sigma V_{2} \Sigma\right)_{13} U_{\alpha, 23}\left(\hat{J}_{2} \otimes J_{1} \otimes \hat{J}_{2}\right) \\
& =\nu_{1}^{-i / 4}\left(\hat{J}_{2} \otimes J_{1} \otimes \hat{J}_{2}\right) U_{\alpha, 23}^{*} \tilde{U}_{\alpha, 21} U_{\alpha, 23}\left(\Sigma V_{2} \Sigma\right)_{13} Z_{21}^{*}\left(\hat{J}_{2} \otimes J_{1} \otimes \hat{J}_{2}\right) \\
& =\nu_{1}^{-i / 4}\left(\hat{J}_{2} \otimes J_{1} \otimes \hat{J}_{2}\right) \tilde{U}_{\alpha, 21}\left(\Sigma V_{2} \Sigma\right)_{13} Z_{21}^{*}\left(\hat{J}_{2} \otimes J_{1} \otimes \hat{J}_{2}\right) \\
& =U_{\alpha, 21} W_{2,13}^{*} U_{\alpha, 21}^{*} U_{\beta, 21}=(\alpha \otimes \iota)\left(W_{2}^{*}\right)_{213} U_{\beta, 21} .
\end{aligned}
$$

This proves our claim.

Defining $\tau_{t}^{\mathrm{m}}:=\tau_{-t}^{1} \otimes \tau_{t}^{2}$, it follows from Lemma 3.6 that for all $\omega \in \mathrm{B}\left(H_{1} \otimes H_{2}\right)_{*}$,

$$
R_{\mathrm{m}} \tau_{-i / 2}^{\mathrm{m}}(\iota \otimes \iota \otimes \omega)\left(W_{\mathrm{m}}\right)=(\iota \otimes \iota \otimes \omega)\left(W_{\mathrm{m}}^{*}\right)
$$

From Lemma 3.6, we also know that $R_{\mathrm{m}}$ and $\tau_{t}^{\mathrm{m}}$ commute. So, we can conclude that $R_{\mathrm{m}}$ is the unitary antipode of $\left(M_{\mathrm{m}}, \Delta_{\mathrm{m}}\right)$ and $\left(\tau_{t}^{\mathrm{m}}\right)$ is its scaling group.

Taking the $i t$-th power of the Radon-Nikodym derivative of $\left(\varphi_{1}\right)_{k_{1}} \otimes \psi_{2}$ w.r.t. $\varphi_{\mathrm{m}}$, we get $\rho_{1}^{i t} \delta_{1}^{-2 i t} \otimes \rho_{2}^{-i t} \delta_{2}^{2 i t}$. These unitaries are group-like because of Proposition 4.2. By uniqueness of the Haar weights, $\varphi_{\mathrm{m}} R_{\mathrm{m}}$ is proportional to $\left(\varphi_{1}\right)_{k_{1}} \otimes \psi_{2}$ and also the Radon-Nikodym derivative of $\varphi_{\mathrm{m}} R_{\mathrm{m}}$ w.r.t. $\varphi_{\mathrm{m}}$ is group-like. Because a non-trivial multiple of a group-like is no longer group-like, we conclude that $\varphi_{\mathrm{m}} R_{\mathrm{m}}=\left(\varphi_{1}\right)_{k_{1}} \otimes \psi_{2}$. So, $\left(\varphi_{1}\right)_{k_{1}} \otimes \psi_{2}$ is indeed the canonical right invariant weight of $\left(M_{\mathrm{m}}, \Delta_{\mathrm{m}}\right)$.

We also conclude that $\delta_{\mathrm{m}}=\rho_{1}^{i t} \delta_{1}^{-2 i t} \otimes \rho_{2}^{-i t} \delta_{2}^{2 i t}$. Because clearly $\varphi_{\mathrm{m}} \tau_{t}^{\mathrm{m}}=\left(\nu_{1} / \nu_{2}\right)^{t} \varphi_{\mathrm{m}}$, we get that $\nu_{\mathrm{m}}=\frac{\nu_{2}}{\nu_{1}}$.

From [14], Proposition 2.5 and from Proposition 4.2 above, we know that $J_{\mathrm{m}}=\lambda_{1}^{i / 4}\left(J_{1} \otimes J_{2}\right)$. Denote by $\hat{J}_{\mathrm{m}}$ the modular conjugation of the canonical left invariant weight on the dual of $\left(M_{\mathrm{m}}, \Delta_{\mathrm{m}}\right)$. Write $U_{\mathrm{m}}:=\hat{J}_{\mathrm{m}} J_{\mathrm{m}}$. Then, we know that

$$
\begin{aligned}
\left(1 \otimes 1 \otimes U_{\mathrm{m}}\right)\left(\Sigma V_{1}^{*} \Sigma\right)_{13} Z_{34}^{*} W_{2,24} Z_{34}\left(1 \otimes 1 \otimes U_{\mathrm{m}}^{*}\right) & =\left(1 \otimes 1 \otimes U_{\mathrm{m}}\right) W_{\mathrm{m}}\left(1 \otimes 1 \otimes U_{\mathrm{m}}^{*}\right) \\
& =\Sigma V_{\mathrm{m}} \Sigma=Z_{34}^{*} W_{1,13}^{*} Z_{34}\left(\Sigma V_{2} \Sigma\right)_{24}
\end{aligned}
$$

Taking slices on the first two legs, it follows that $U_{\mathrm{m}}(x \otimes 1) U_{\mathrm{m}}^{*}=Z^{*}\left(u_{1} x u_{1}^{*} \otimes 1\right) Z$ for all $x \in \hat{M}_{1}^{\prime}$, where we use the notation $u_{i}=\hat{J}_{i} J_{i}$. Applying $\operatorname{Ad}\left(1 \otimes 1 \otimes U_{\mathrm{m}}^{*}\right)$ to Equation (5.3), realizing that $U_{\mathrm{m}}$ and $U_{\mathrm{m}}^{*}$ only differ up to a scalar and again taking slices, we also conclude that $U_{\mathrm{m}}(1 \otimes y) U_{\mathrm{m}}^{*}=Z^{*}\left(1 \otimes u_{2} y u_{2}^{*}\right) Z$ for all $y \in \hat{M}_{2}^{\prime}$. We conclude that $\operatorname{Ad} U_{\mathrm{m}}=\operatorname{Ad}\left(Z^{*}\left(u_{1} \otimes u_{2}\right)\right)$ on $\hat{M}_{1}^{\prime} \otimes \hat{M}_{2}^{\prime}$. As we know $J_{\mathrm{m}}$, it follows that

$$
\hat{J}_{\mathrm{m}} z \hat{J}_{\mathrm{m}}=Z^{*}\left(\hat{J}_{1} \otimes \hat{J}_{1}\right) z\left(\hat{J}_{1} \otimes \hat{J}_{2}\right) Z,
$$

for all $z \in \hat{M}_{1}^{\prime} \otimes \hat{M}_{2}^{\prime}$. If next $z \in M_{1} \otimes M_{2}$, the left hand side equals $R_{\mathrm{m}}\left(z^{*}\right)$, but the right hand side as well because $\mathrm{m}^{-1}\left(R_{1} \otimes R_{2}\right)\left(z^{*}\right)=R_{\mathrm{m}}\left(z^{*}\right)$. Because their quotient commutes with both $\hat{M}_{1}^{\prime} \otimes \hat{M}_{2}^{\prime}$ and $M_{1} \otimes M_{2}$, we conclude that $\hat{J}_{\mathrm{m}}$ and $Z^{*}\left(\hat{J}_{1} \otimes \hat{J}_{2}\right)$ only differ up to a scalar.

Because $\hat{J}_{\mathrm{m}}^{2}=1$, we also get $\left(Z^{*}\left(\hat{J}_{1} \otimes \hat{J}_{2}\right)\right)^{2}=1$. Writing this out, it follows that

$$
\left(J_{1} \otimes J_{2}\right) J \hat{J}=\hat{J} J\left(J_{1} \otimes J_{2}\right) .
$$

Using this commutation relation, we arrive at

$$
J_{\mathrm{m}} Z^{*}\left(\hat{J}_{1} \otimes \hat{J}_{2}\right)=\lambda_{1}^{i / 2}\left(\frac{\nu_{1}}{\nu_{2}}\right)^{i / 4} Z^{*}\left(\hat{J}_{1} \otimes \hat{J}_{2}\right) J_{\mathrm{m}}
$$

On the other hand,

$$
J_{\mathrm{m}} \hat{J}_{\mathrm{m}}=\nu_{\mathrm{m}}^{-i / 4} \hat{J}_{\mathrm{m}} J_{\mathrm{m}}=\left(\frac{\nu_{1}}{\nu_{2}}\right)^{i / 4} \hat{J}_{\mathrm{m}} J_{\mathrm{m}}
$$

We conclude that

$$
\hat{J}_{\mathrm{m}}=\lambda_{1}^{i / 4} Z^{*}\left(\hat{J}_{1} \otimes \hat{J}_{2}\right)=\lambda_{1}^{i / 4}\left(J_{1} \hat{J}_{1} \otimes J_{2} \hat{J}_{2}\right) \hat{J} J\left(\hat{J}_{1} \otimes \hat{J}_{2}\right)=\lambda_{1}^{i / 4}\left(\hat{J}_{1} \otimes \hat{J}_{2}\right) J \hat{J}\left(\hat{J}_{1} J_{1} \otimes \hat{J}_{2} J_{2}\right)
$$


Because we know $\left(\tau_{t}^{\mathrm{m}}\right)$ and the GNS-construction for $\varphi_{\mathrm{m}}$, we immediately get that $P_{\mathrm{m}}=P_{1}^{-1} \otimes P_{2}$. Because

$$
\nabla_{\mathrm{m}}^{i t}=\nabla_{1}^{i t} \otimes \nabla_{2}^{i t} k_{2}^{i t} J_{2} k_{2}^{i t} J_{2} \quad \text { and } \quad \nabla_{\mathrm{m}}^{i t}=\nabla_{1}^{i t} k_{1}^{i t} J_{1} k_{1}^{i t} J_{1} \otimes \nabla_{2}^{i t},
$$

where $\nabla_{1}, \nabla_{2}$ and $\nabla_{\mathrm{m}}$ denote the modular operators of $\psi_{1}, \psi_{2}$ and $\psi_{\mathrm{m}}$ respectively, it is easy to find, out of Proposition 2.4 in [16], the formula

$$
\hat{\delta}_{\mathrm{m}}^{i t}=\hat{J}_{1} \hat{\delta}_{1}^{-i t} \hat{J}_{1} k_{1}^{-i t} J_{1} k_{1}^{-i t} J_{1} \otimes \hat{\delta}_{2}^{i t} .
$$

But, from Proposition 4.2, it follows that $\hat{J}\left(1 \otimes \hat{\delta}_{2}^{i t}\right) \hat{J}=k_{1}^{-i t} J_{1} k_{1}^{-i t} J_{1} \otimes \hat{J}_{2} \hat{\delta}_{2}^{i t} \hat{J}_{2}$. Because $1 \otimes \hat{\delta}_{2}^{i t}$ is a group-like unitary of $(\hat{M}, \hat{\Delta})$, we know that $J\left(1 \otimes \hat{\delta}_{2}^{i t}\right) J=1 \otimes \hat{\delta}_{2}^{i t}$ and so $\hat{\delta}_{\mathrm{m}}^{i t}=\left(\hat{J}_{1} \hat{\delta}_{1}^{-i t} \hat{J}_{1} \otimes 1\right) Z^{*}\left(1 \otimes \hat{\delta}_{2}^{i t}\right) Z$. The formula for $\hat{J}_{\mathrm{m}} \hat{\delta}_{\mathrm{m}}^{i t} \hat{J}_{\mathrm{m}}$ is proven analogously.

\section{Actions of double crossed products and bicrossed products}

Suppose that $\rho: N \rightarrow M_{\mathrm{m}} \otimes N$ is an action of the double crossed product $\left(M_{\mathrm{m}}, \Delta_{\mathrm{m}}\right)$ on the von Neumann algebra $N$. We will show how to construct another von Neumann algebra $L$ with an action of the bicrossed product $(M, \Delta)$ and we show that the strict outerness of one action is equivalent to the strict outerness of the other action. As a definition of strict outerness, we take Definition 5.5 in [13]: $M_{\mathrm{m} \rho} \ltimes N \cap \rho(N)^{\prime}=\mathbb{C}$. In [12], the second author proves that every l.c. quantum group can act strictly outerly in this strong sense on a factor.

It is clear that we have morphisms $\left(M_{\mathrm{m}}, \Delta_{\mathrm{m}}\right) \rightarrow\left(M_{1}, \Delta_{1}^{\mathrm{op}}\right)$ and $\left(M_{\mathrm{m}}, \Delta_{\mathrm{m}}\right) \rightarrow\left(M_{2}, \Delta_{2}\right)$. Although these morphisms do not exist on the von Neumann algebra level, they can nevertheless be used to restrict the action $\rho$ to an action of $\left(M_{1}, \Delta_{1}^{\text {op }}\right)$, resp. $\left(M_{2}, \Delta_{2}\right)$. We briefly explain how to do this.

In the von Neumann algebra language, a morphism appears rather as a special type of action (the restricted left or right translation), as was pointed out by J. Kustermans in [7]. We clearly have a left action $\zeta_{1}$ : $M_{\mathrm{m}} \rightarrow M_{1} \otimes M_{\mathrm{m}}: \zeta_{1}=\Delta_{1}^{\mathrm{op}} \otimes \iota$ of $\left(M_{1}, \Delta_{1}^{\mathrm{op}}\right)$ on $M_{\mathrm{m}}$ satisfying $\left(\iota \otimes \Delta_{\mathrm{m}}\right) \zeta_{1}=\left(\zeta_{1} \otimes \iota \otimes \iota\right) \Delta_{\mathrm{m}}$. Analogously, we have the right action $\iota \otimes \Delta_{2}: M_{\mathrm{m}} \rightarrow M_{\mathrm{m}} \otimes M_{2}$, which can be composed with the unitary antipodes to yield the left action of $\left(M_{2}, \Delta_{2}\right)$ on $M_{\mathrm{m}}$ given by $\zeta_{2}: M_{\mathrm{m}} \rightarrow M_{2} \otimes M_{\mathrm{m}}: \zeta_{2}=\sigma_{12} \mathrm{~m}_{12}\left(\iota \otimes \Delta_{2}\right)$.

This allows us to define the action $\eta_{1}: N \rightarrow M_{1} \otimes N$ of $\left(M_{1}, \Delta_{1}^{\text {op }}\right)$ on $N$ such that $(\iota \otimes \rho) \eta_{1}=\left(\zeta_{1} \otimes \iota\right) \rho$ and the action $\eta_{2}: N \rightarrow M_{2} \otimes N$ of $\left(M_{2}, \Delta_{2}\right)$ on $N$ such that $(\iota \otimes \rho) \eta_{2}=\left(\zeta_{2} \otimes \iota\right) \rho$. Denote

$$
L:=M_{1 \eta_{1}} \ltimes N=\left(\eta_{1}(N) \cup \hat{M}_{1}^{\prime} \otimes 1\right)^{\prime \prime} .
$$

It is easy to check that $\left(\iota \otimes \eta_{2}\right) \eta_{1}=\rho$ and applying $\iota \otimes \eta_{2}$ to $L$, we arrive at another representation of $L$ : $\left(\rho(N) \cup \hat{M}_{1}^{\prime} \otimes 1 \otimes 1\right)^{\prime \prime}$. This shows how $L$ is an intermediate von Neumann algebra

$$
N \hookrightarrow L \hookrightarrow M_{\mathrm{m} \rho} \ltimes N
$$

On $L$, we will define a natural action $\eta$ of the bicrossed product $(M, \Delta)$ such that

$$
N \hookrightarrow L \hookrightarrow M_{\mathrm{m} \rho} \ltimes N \hookrightarrow M_{\eta} \ltimes L \hookrightarrow \hat{M}_{\mathrm{m} \hat{\rho}} \ltimes\left(M_{\mathrm{m} \rho} \ltimes N\right) .
$$

Proposition 6.1. There exists a unique action $\eta: L \rightarrow M \otimes L$ of $(M, \Delta)$ on $L$ such that

$$
\eta\left(\eta_{1}(x)\right)=\left(\alpha \otimes \eta_{1}\right) \eta_{2}(x) \quad \text { for all } x \in N \quad \eta\left(\hat{J}_{1} y \hat{J}_{1} \otimes 1\right)=\left(\hat{J} \otimes \hat{J}_{1}\right) \mu(y)\left(\hat{J} \otimes \hat{J}_{1}\right) \otimes 1 \quad \text { for all } y \in \hat{M}_{1},
$$

where $\mu: \hat{M}_{1} \rightarrow M \otimes \hat{M}_{1}$ is the left action of $\left(M, \Delta^{\mathrm{op}}\right)$ on $\hat{M}_{1}$ defined by $\mu(y) \otimes 1=\Delta^{\mathrm{op}}(y \otimes 1)$.

Moreover, the action $\eta$ is strictly outer if and only if the action $\rho$ is strictly outer. Finally, the inclusion of Equation (6.1) holds.

Proof. During the proof, we replace $L$ by its representation $\left(\rho(N) \cup \hat{M}_{1}^{\prime} \otimes 1 \otimes 1\right)^{\prime \prime}$ in $\mathrm{B}\left(H_{1} \otimes H_{2}\right) \otimes N$. Define for $z \in L$,

$$
\eta(z)=\mathcal{U}_{123}(\iota \otimes \iota \otimes \rho)(z) \mathcal{U}_{123}^{*} \quad \text { where } \quad \mathcal{U}=\left(\hat{J} \otimes \hat{J}_{1}\right)(\beta \otimes \iota)\left(W_{1}\right)\left(\hat{J}_{1} \otimes \hat{J}_{2} \otimes \hat{J}_{1}\right)
$$


Represent $N$ on its standard Hilbert space, with anti-unitary $J_{N}$ and use the notation

$$
\tilde{\rho}(y)=\left(\hat{J}_{1} \otimes \hat{J}_{2} \otimes J_{N}\right) \rho\left(J_{N} y J_{N}\right)\left(\hat{J}_{1} \otimes \hat{J}_{2} \otimes J_{N}\right) \quad \text { for } \quad y \in N^{\prime} .
$$

Let $x \in N$. We have

$$
\begin{aligned}
& \eta(\rho(x))=\mathcal{U}_{123}\left(\Delta_{\mathrm{m}} \otimes \iota\right) \rho(x) \mathcal{U}_{123}^{*} \\
& =\left(\hat{J} \otimes \hat{J}_{1} \otimes \hat{J}_{2} \otimes J_{N}\right)(\beta \otimes \iota)\left(W_{1}\right)_{123}\left(\sigma_{23} \mathrm{~m}_{23}^{-1}\left(\Delta_{1} \otimes \Delta_{2}^{\mathrm{op}} \otimes \iota\right) \tilde{\rho}\left(J_{N} x J_{N}\right)\right)(\beta \otimes \iota)\left(W_{1}^{*}\right)_{123}\left(\hat{J} \otimes \hat{J}_{1} \otimes \hat{J}_{2} \otimes J_{N}\right) \\
& =\left(\hat{J} \otimes \hat{J}_{1} \otimes \hat{J}_{2} \otimes J_{N}\right)(\alpha \otimes \iota \otimes \iota \otimes \iota)\left(\sigma_{12} \mathrm{~m}_{12}^{-1}\left(\iota \otimes \Delta_{2}^{\mathrm{op}} \otimes \iota\right) \tilde{\rho}\left(J_{N} x J_{N}\right)\right)\left(\hat{J} \otimes \hat{J}_{1} \otimes \hat{J}_{2} \otimes J_{N}\right) \\
& =(\alpha \otimes \iota \otimes \iota \otimes \iota)\left(\sigma_{12} \mathrm{~m}_{12}\left(\iota \otimes \Delta_{2} \otimes \iota\right) \rho(x)\right)=(\alpha \otimes \rho) \eta_{2}(x) .
\end{aligned}
$$

On the other hand, it is obvious that $\eta\left(\hat{J}_{1} y \hat{J}_{1} \otimes 1 \otimes 1\right)=\left(\hat{J} \otimes \hat{J}_{1}\right) \mu(y)\left(\hat{J} \otimes \hat{J}_{1}\right) \otimes 1 \otimes 1$ for $y \in \hat{M}_{1}$. Then, it is also clear that $\eta: L \rightarrow M \otimes L$ and that $\eta$ is an action of $(M, \Delta)$ on $L$.

By definition, $M_{\eta} \ltimes L=(\eta(L) \cup \hat{M} \otimes 1 \otimes 1 \otimes 1)^{\prime \prime}$, but using $\mathcal{U}$, we easily identify

$$
M_{\eta} \ltimes L=\left(L \cup\left(\hat{J}_{1} \otimes \hat{J}_{2}\right) \hat{J} \hat{M} \hat{J}\left(\hat{J}_{1} \otimes \hat{J}_{2}\right) \otimes 1\right)^{\prime \prime} \subset \mathrm{B}\left(H_{1} \otimes H_{2}\right) \otimes N .
$$

In this way, we see that the inclusion of Equation (6.1) is given by

$$
\rho(N) \subset L \subset\left(\rho(N) \cup \hat{M}_{\mathrm{m}} \otimes 1\right)^{\prime \prime} \subset\left(L \cup\left(\hat{J}_{1} \otimes \hat{J}_{2}\right) \hat{J} \hat{M} \hat{J}\left(\hat{J}_{1} \otimes \hat{J}_{2}\right) \otimes 1\right)^{\prime \prime} \subset \mathrm{B}\left(H_{1} \otimes H_{2}\right) \otimes N .
$$

Suppose first that $\rho$ is strictly outer and let $z \in\left(M_{\eta} \ltimes L\right) \cap L^{\prime}$. Then, we observe that $z \in \mathrm{B}\left(H_{1} \otimes H_{2}\right) \otimes$ $N \cap \rho(N)^{\prime}$, which equals $M_{1}^{\prime} \otimes M_{2}^{\prime} \otimes 1$ by the strict outerness of $\rho$. So, $z=y \otimes 1$ with $y \in M_{1}^{\prime} \otimes M_{2}^{\prime}$. But $z$ also commutes with $\hat{M}_{1}^{\prime} \otimes 1 \otimes 1$, which yields $z=1 \otimes a \otimes 1$ with $a \in M_{2}^{\prime}$. Because

$$
\left(\iota \otimes \iota \otimes \eta_{2}\right) \rho=\left(\iota \otimes \iota \otimes \eta_{2}\right)\left(\iota \otimes \eta_{2}\right) \eta_{1}=\left(\iota \otimes \Delta_{2} \otimes \iota\right) \rho
$$

we see that $\left(\iota \otimes \iota \otimes \eta_{2}\right)(z)=V_{2,23} z_{124} V_{2,23}^{*}$ for all $z \in M_{\eta} \ltimes L$. So, $a \in \hat{M}_{2}^{\prime}$ and hence, $a \in \mathbb{C}$. We get that $\eta$ is strictly outer.

Suppose next that $\eta$ is strictly outer and let $z \in \mathrm{B}\left(H_{1} \otimes H_{2}\right) \otimes N \cap\left(M_{\mathrm{m} \rho} \ltimes N\right)^{\prime}$. We claim that,

$$
\mathcal{U}_{123}(\iota \otimes \iota \otimes \rho)(y) \mathcal{U}_{123}^{*} \in \mathrm{B}\left(H_{1} \otimes H_{2}\right) \otimes L \quad \text { for all } \quad y \in \mathrm{B}\left(H_{1} \otimes H_{2}\right) \otimes N .
$$

When $y \in \rho(N)$, this is clear and when $y \in \mathrm{B}\left(H_{1} \otimes H_{2}\right) \otimes 1$, it follows because $\mathcal{U} \in \mathrm{B}\left(H_{1} \otimes H_{2}\right) \otimes \hat{M}_{1}^{\prime}$. Because $\rho$ is an action, we know that

$$
\mathrm{B}\left(H_{1} \otimes H_{2}\right) \otimes N=\left(\rho(N) \cup \mathrm{B}\left(H_{1} \otimes H_{2}\right) \otimes 1\right)^{\prime \prime}
$$

and our claim is proven. So, we find in particular that $\mathcal{U}_{123}(\iota \otimes \iota \otimes \rho)(z) \mathcal{U}_{123}^{*} \in \mathrm{B}\left(H_{1} \otimes H_{2}\right) \otimes L \cap \eta(L)^{\prime}$. From the strict outerness of $\eta$, it follows that $\mathrm{B}\left(H_{1} \otimes H_{2}\right) \otimes L \cap \eta(L)^{\prime}=M^{\prime} \otimes 1 \otimes 1 \otimes 1$ and we can take $a \in M^{\prime}$ such that

$$
(\iota \otimes \iota \otimes \rho)(z)=\mathcal{U}_{123}^{*}(a \otimes 1 \otimes 1 \otimes 1) \mathcal{U}_{123}
$$

But, $\rho(N) \subset M_{1} \otimes M_{2} \otimes N$, while the last leg of $\mathcal{U}$ sits in $\hat{M}_{1}^{\prime}$. Hence, $z=y \otimes 1$ for some $y \in \mathrm{B}\left(H_{1} \otimes H_{2}\right)$. Writing $x=\left(\hat{J}_{1} \otimes \hat{J}_{2}\right) y\left(\hat{J}_{1} \otimes \hat{J}_{2}\right)$ and $b=\hat{J} a \hat{J} \in M^{\prime}$, we get

$$
(\beta \otimes \iota)\left(W_{1}\right)(x \otimes 1)(\beta \otimes \iota)\left(W_{1}^{*}\right)=b \otimes 1 .
$$

If we write $\zeta(c)=(\beta \otimes \iota)\left(W_{1}\right)(c \otimes 1)(\beta \otimes \iota)\left(W_{1}^{*}\right)$ for $c \in \mathrm{B}\left(H_{1} \otimes H_{2}\right)$, we see that $\zeta: \mathrm{B}\left(H_{1} \otimes H_{2}\right) \rightarrow$ $\mathrm{B}\left(H_{1} \otimes H_{2}\right) \otimes \hat{M}_{1}$ is a right action of $\left(\hat{M}_{1}, \hat{\Delta}_{1}^{\text {op }}\right)$ on $\mathrm{B}\left(H_{1} \otimes H_{2}\right)$. Because $\zeta(x)=b \otimes 1$, we find that $\zeta(b) \otimes 1=(\zeta \otimes \iota) \zeta(x)=\left(\iota \otimes \iota \otimes \hat{\Delta}_{1}^{\mathrm{op}}\right) \zeta(x)=b \otimes 1 \otimes 1$. Hence, $\zeta(b)=b \otimes 1$ and $x=b$. We arrive at the conclusion that $x \in M^{\prime} \cap \beta\left(M_{1}\right)^{\prime}=\alpha\left(M_{2}\right)^{\prime} \cap\left(\hat{M}_{1} \otimes 1\right)^{\prime} \cap \beta\left(M_{1}\right)^{\prime}=M_{1}^{\prime} \otimes M_{2}^{\prime} \cap\left(\hat{M}_{1} \otimes 1\right)^{\prime}=1 \otimes M_{2}^{\prime}$. This gives us that $z=1 \otimes d \otimes 1$ with $d \in M_{2}^{\prime}$. Because $z \in\left(M_{\mathrm{m} \rho} \propto N\right)^{\prime}, 1 \otimes d$ must commute with $Z^{*}\left(1 \otimes \hat{M}_{2}\right) Z$. By Theorem 5.3, $Z^{*}$ equals $\left(\hat{J}_{1} J_{1} \otimes 1\right) U_{\beta}^{*} J\left(J_{1} \otimes J_{2}\right)$, up to a scalar. The first part belongs to $\mathrm{B}\left(H_{1}\right) \otimes M_{2}$ and we find that $1 \otimes d$ must commute with $J\left(1 \otimes \hat{M}_{2}\right) J$. This means that $\alpha\left(J_{2} d J_{2}\right)$ commutes with $1 \otimes \hat{M}_{2}$. Hence, $\alpha\left(J_{2} d J_{2}\right) \in M_{1} \otimes 1$. Because $\alpha$ is an action of $\left(M_{1}, \Delta_{1}\right)$ on $M_{2}$, it follows that $J_{2} d J_{2} \in \mathbb{C}$. So, $z \in \mathbb{C}$ and we are done. 


\section{A characterization of double crossed products}

In Definition 2.3, we defined a matching $m$ of l.c. quantum groups $\left(M_{1}, \Delta_{1}\right),\left(M_{2}, \Delta_{2}\right)$ and we constructed the associated double crossed product $\left(M_{\mathrm{m}}, \Delta_{\mathrm{m}}\right)$ as a l.c. quantum group in Section 5 . It is clear that the mappings $\Delta_{1}^{\mathrm{op}} \otimes \iota$ and $\iota \otimes \Delta_{2}$ define, respectively, a left action of $\left(M_{1}, \Delta_{1}^{\mathrm{op}}\right)$ and a right action of $\left(M_{2}, \Delta_{2}\right)$ on the von Neumann algebra $M_{\mathrm{m}} \cong M_{1} \otimes M_{2}$. In fact, these actions are precisely the actions that correspond to the natural morphisms from $\left(M_{\mathrm{m}}, \Delta_{\mathrm{m}}\right)$ to $\left(M_{1}, \Delta_{1}^{\mathrm{op}}\right)$ and $\left(M_{2}, \Delta_{2}\right)$.

We claim that this characterizes double crossed products: whenever we have a l.c. quantum group $(N, \Gamma)$ and morphisms from $(N, \Gamma)$ to $\left(M_{1}, \Delta_{1}^{\mathrm{op}}\right)$ and $\left(M_{2}, \Delta_{2}\right)$, such that $N$, equipped with the naturally associated left action of $\left(M_{1}, \Delta_{1}^{\text {op }}\right)$ and right action of $\left(M_{2}, \Delta_{2}\right)$, is isomorphic to $M_{1} \otimes M_{2}$ as an $M_{1}, M_{2}$-bicomodule, we get that $(N, \Gamma)$ is a double crossed product.

We can interpret this in the classical, commutative case as follows. We are given l.c. groups $G, G_{1}$ and $G_{2}$. We suppose that $G_{1}^{\text {op }}$ acts on the left and $G_{2}$ on the right on $G$ such that, as a measure space, $G$ is isomorphic to $G_{1} \times G_{2}$ where the isomorphism intertwines the actions of $G_{1}^{\text {op }}$ and $G_{2}$ on $G$ with the natural actions of $G_{1}^{\text {op }}$ and $G_{2}$ on $G_{1} \times G_{2}$. So, we assume in fact that $G_{1}^{\text {op }}$ and $G_{2}$ are closed subgroups of $G$ and that there exists an element $x \in G$ such that the map $G_{1} \times G_{2} \rightarrow G:(g, s) \mapsto g x s$ is a measure class isomorphism. Then, it is clear that, considering $x^{-1} G_{1} x$ and $G_{2}$ as closed subgroups of $G$, we arrive at a matched pair (in the sense of [3], Definition 3.1) of l.c. groups with $G$ as the double crossed product. It is obvious that it is really necessary to allow for the presence of $x \in G$ and this explains the presence of the group-like unitary $u \in \hat{N}$ in Proposition 7.1.

Proposition 7.1. Let $(N, \Gamma),\left(M_{1}, \Delta_{1}\right)$ and $\left(M_{2}, \Delta_{2}\right)$ be l.c. quantum groups. Suppose that there are morphisms from $(N, \Gamma)$ to $\left(M_{1}, \Delta_{1}^{\mathrm{op}}\right)$ and $\left(M_{2}, \Delta_{2}\right)$ respectively, with associated actions $\zeta_{1}: N \rightarrow M_{1} \otimes N$, $\zeta_{2}: N \rightarrow N \otimes M_{2}$ of $\left(M_{1}, \Delta_{1}^{\mathrm{op}}\right)$ on the left and $\left(M_{2}, \Delta_{2}\right)$ on the right on $N$ (in the sense of Proposition 12.1 in [7]). Suppose that there exists an isomorphism of von Neumann algebras $\pi: N \rightarrow M_{1} \otimes M_{2}$ intertwining $\zeta_{1}$ with $\Delta_{1}^{\mathrm{op}} \otimes \iota$ and $\zeta_{2}$ with $\iota \otimes \Delta_{2}$.

Then, there exists a matching $\mathrm{m}: M_{1} \otimes M_{2} \rightarrow M_{1} \otimes M_{2}$ and a group-like unitary $u \in \hat{N}$ such that $\pi \circ \operatorname{Ad} u:$ $N \rightarrow M_{1} \otimes M_{2}$ is an isomorphism between the l.c. quantum groups $(N, \Gamma)$ and the double crossed product $\left(M_{\mathrm{m}}, \Delta_{\mathrm{m}}\right)$.

Proof. Denote by $W_{N}$ the left regular representation of $(N, \Gamma)$. Because the actions $\zeta_{1}, \zeta_{2}$ are defined by morphisms, we know that

$$
\begin{aligned}
& \left(\zeta_{1} \otimes \iota\right) \Gamma=(\iota \otimes \Gamma) \zeta_{1}, \quad\left(\iota \otimes \zeta_{2}\right) \Gamma=(\Gamma \otimes \iota) \zeta_{2} \\
& \left(\zeta_{1} \otimes \iota\right)\left(W_{N}\right)=X_{1,13} W_{N, 23}, \quad\left(\zeta_{2} \otimes \iota\right)\left(W_{N}\right)=W_{N, 13} X_{2,23}
\end{aligned}
$$

where $X_{1} \in M_{1} \otimes \hat{N}$ is a corepresentation of $\left(M_{1}, \Delta_{1}^{\text {op }}\right)$ and $X_{2} \in M_{2} \otimes \hat{N}$ is a corepresentation of $\left(M_{2}, \Delta_{2}\right)$. Defining $\tilde{W}_{N}=(\pi \otimes \iota)\left(W_{N}\right) \in M_{1} \otimes M_{2} \otimes \hat{N}$, we deduce that

$$
\left(\Delta_{1}^{\mathrm{op}} \otimes \iota \otimes \iota\right)\left(\tilde{W}_{N}\right)=X_{1,14} \tilde{W}_{N, 234}, \quad\left(\iota \otimes \Delta_{2} \otimes \iota\right)\left(\tilde{W}_{N}\right)=\tilde{W}_{N, 124} X_{2,34}
$$

It then follows that $X_{1,13}^{*} \tilde{W}_{N}$ is invariant under $\Delta_{1}^{\mathrm{op}} \otimes \iota \otimes \iota$ and we find $Y \in M_{2} \otimes \hat{N}$ such that $\tilde{W}_{N}=X_{1,13} Y_{23}$. Using next the formula for $\left(\iota \otimes \Delta_{2} \otimes \iota\right)\left(\tilde{W}_{N}\right)$, we get $\left(\Delta_{2} \otimes \iota\right)(Y)=Y_{13} X_{2,23}$. Hence, $Y X_{2}^{*}$ is invariant under $\Delta_{2} \otimes \iota$ and we find a unitary $u \in \hat{N}$ such that

$$
\tilde{W}_{N}=X_{1,13}(1 \otimes 1 \otimes u) X_{2,23}
$$

We know that $\left(\iota \otimes \iota \otimes \hat{\Delta}_{N}\right)\left(\tilde{W}_{N}\right)=\tilde{W}_{N, 124} \tilde{W}_{N, 123}$, from which we conclude that $\left(\iota \otimes \hat{\Delta}_{N}\right)\left(X_{i}\right)=X_{i, 13} X_{i, 12}$ for $i=1,2$. Writing this out, it follows that $\hat{\Delta}(u)=u \otimes u$.

The equation $\hat{\Delta}(u)=u \otimes u$ gives us that $(\operatorname{Ad} u \otimes \iota)\left(W_{N}\right)=\left(1 \otimes u^{*}\right) W_{N}$. From Equation (7.1), it is clear that $\zeta_{2}$ intertwines $\operatorname{Ad} u$ and $\operatorname{Ad}(u \otimes 1)$, while defining $\tilde{\zeta}_{1}=\left(\iota \otimes \operatorname{Ad} u^{*}\right) \circ \zeta_{1} \circ \operatorname{Ad} u$ and $\tilde{X}_{1}=\left(1 \otimes u^{*}\right) X_{1}(1 \otimes u)$, we get

$$
\left(\tilde{\zeta}_{1} \otimes \iota\right)\left(W_{N}\right)=\tilde{X}_{1,13} W_{N, 23}
$$


Finally, we put $\tilde{\pi}=\pi \circ \operatorname{Ad} u$ and observe that $(\tilde{\pi} \otimes \iota)\left(W_{N}\right)=\tilde{X}_{1,13} X_{2,23}$. Also, $\tilde{\pi}$ intertwines $\tilde{\zeta}_{1}$ with $\Delta_{1}^{\mathrm{op}} \otimes \iota$ and $\zeta_{2}$ with $\iota \otimes \Delta_{2}$. As a conclusion, we see that changing the morphism from $(N, \Gamma)$ to $\left(M_{1}, \Delta_{1}^{\mathrm{op}}\right)$ by using $u$, we may in fact suppose from the beginning that $u=1$ and now prove the existence of a matching $\mathrm{m}: M_{1} \otimes M_{2} \rightarrow M_{1} \otimes M_{2}$ such that $\pi$ is an isomorphism between the l.c. quantum groups $(N, \Gamma)$ and $\left(M_{\mathrm{m}}, \Delta_{\mathrm{m}}\right)$.

So, we assume that $u=1$ and get $\tilde{W}_{N}=X_{1,13} X_{2,23}$. Because a morphism commutes with the antipode (Remark 12.1 in [7]), we know that $\zeta_{1} \tau_{t}^{N}=\left(\tau_{-t}^{1} \otimes \tau_{t}^{N}\right) \zeta_{1}$ and $\zeta_{2} \tau_{t}^{N}=\left(\tau_{t}^{N} \otimes \tau_{t}^{2}\right) \zeta_{2}$. Combining this with Equation (7.1), we get that $X_{1}$ is invariant under $\tau_{-t}^{1} \otimes \hat{\tau}_{t}^{N}$, while $X_{2}$ is invariant under $\tau_{t}^{2} \otimes \hat{\tau}_{t}^{N}$. Because $\tilde{W}_{N}=X_{1,13} X_{2,23}$, it follows that $\pi \tau_{t}^{N}=\left(\tau_{-t}^{1} \otimes \tau_{t}^{2}\right) \pi$. Moreover, because $X_{1}$ and $X_{2}$ are corepresentations, it follows that, for all $\omega \in \hat{N}_{*}$, the element $y:=(\iota \otimes \iota \otimes \omega)\left(X_{1,13} X_{2,23}\right) \in D\left(S_{1}^{-1} \otimes S_{2}\right)$ and

$$
\left(S_{1}^{-1} \otimes S_{2}\right)(y)=(\iota \otimes \iota \otimes \omega)\left(X_{1,13}^{*} X_{2,23}^{*}\right) .
$$

Define $\mathrm{m}:=\left(R_{1} \otimes R_{2}\right) \pi R_{N} \pi^{-1}$, use that $S_{N}(\iota \otimes \omega)\left(W_{N}\right)=(\iota \otimes \omega)\left(W_{N}^{*}\right)$ and combine this with the formulas above, to conclude that

$$
(\mathrm{m} \otimes \iota)\left(X_{1,13} X_{2,23}\right)=X_{2,23} X_{1,13} .
$$

It follows that $\left((\iota \otimes \sigma \mathrm{m} \otimes \iota)\left(\Delta_{1}^{\mathrm{op}} \otimes \Delta_{2}\right) \otimes \iota\right)\left(\tilde{W}_{N}\right)=\tilde{W}_{N, 125} \tilde{W}_{N, 345}$ and hence, $(\iota \otimes \sigma \mathrm{m} \otimes \iota)\left(\Delta_{1}^{\mathrm{op}} \otimes \Delta_{2}\right) \pi=(\pi \otimes \pi) \Gamma$. We also verify easily that

$$
\begin{array}{ll} 
& \left(\left(\Delta_{1} \otimes \iota\right) \mathrm{m} \otimes \iota\right)\left(X_{1,13} X_{2,23}\right)=X_{2,34} X_{1,24} X_{1,14} \\
\text { and } \quad \mathrm{m}_{23} \mathrm{~m}_{13}\left(\Delta_{1} \otimes \iota \otimes \iota\right)\left(X_{1,13} X_{2,23}\right)=\mathrm{m}_{23} \mathrm{~m}_{13}\left(X_{1,24} X_{1,14} X_{2,34}\right)=X_{2,34} X_{1,24} X_{1,14} .
\end{array}
$$

Because the slices of $\tilde{W}_{N}$ are dense in $\pi(N)=M_{1} \otimes M_{2}$, we conclude that $\left(\Delta_{1} \otimes \iota\right) \mathrm{m}=\mathrm{m}_{23} \mathrm{~m}_{13}\left(\Delta_{1} \otimes \iota\right)$. Completely analogously, we verify that $\left(\iota \otimes \Delta_{2}\right) \mathrm{m}=\mathrm{m}_{13} \mathrm{~m}_{12}\left(\iota \otimes \Delta_{2}\right)$. Hence, $\mathrm{m}$ is a matching and above, we have seen already that $\pi: N \rightarrow M_{\mathrm{m}}$ is an isomorphism of l.c. quantum groups.

\section{Example: generalized quantum doubles}

Let $\left(M_{1}, \Delta_{1}\right)$ and $\left(M_{2}, \Delta_{2}\right)$ be l.c. quantum groups. Suppose that $\mathcal{Z} \in M_{1} \otimes M_{2}$ is a bicharacter in the sense that

$$
\left(\Delta_{1} \otimes \iota\right)(\mathcal{Z})=\mathcal{Z}_{23} \mathcal{Z}_{13} \quad \text { and } \quad\left(\iota \otimes \Delta_{2}\right)(\mathcal{Z})=\mathcal{Z}_{13} \mathcal{Z}_{12}
$$

(We see that, in fact, $\mathcal{Z}^{*}$ is the bicharacter.) Then, we can define a matching $\mathrm{m}$ as the inner isomorphism:

$$
\mathrm{m}(x)=\mathcal{Z} x \mathcal{Z}^{*} \quad \text { for all } \quad x \in M_{1} \otimes M_{2} .
$$

It is clear that $\mathrm{m}$ is a matching of $\left(M_{1}, \Delta_{1}\right)$ and $\left(M_{2}, \Delta_{2}\right)$. Hence, we have an associated bicrossed product $(M, \Delta)$ and double crossed product $\left(M_{\mathrm{m}}, \Delta_{\mathrm{m}}\right)$, as above.

A particular case is that of the quantum double: let $\left(N, \Delta_{N}\right)$ be a l.c. quantum group and put $\left(M_{1}, \Delta_{1}\right)=$ $\left(N, \Delta_{N}^{\mathrm{op}}\right),\left(M_{2}, \Delta_{2}\right)=\left(\hat{N}, \hat{\Delta}_{N}\right)$ and $\mathcal{Z}=W_{N}$. The associated double crossed products is the quantum double of $\left(N, \Delta_{N}\right)$. Such a quantum double construction in the framework of l.c. quantum groups (or rather, Woronowicz algebras) has been done for the first time by T. Yamanouchi [23].

For this reason, we call the double crossed product associated with an inner matching as in Equation (8.1) a generalized quantum double.

Suppose for the rest of this section that we have a bicharacter $\mathcal{Z}$ as above and an inner matching $\mathrm{m}$ defined by Equation (8.1).

Proposition 8.1. Through the isomorphism $\operatorname{Ad} \mathcal{Z}^{*}$, the bicrossed product $(M, \Delta)$ is isomorphic with the tensor product $\left(\hat{M}_{1} \otimes M_{2},(\iota \otimes \sigma \otimes \iota)\left(\hat{\Delta}_{1} \otimes \Delta_{2}\right)\right)$.

The ingredients of the double crossed product (i.e., the generalized quantum double) are given by Theorem 5.3, combined with the formulas: 
- $J=\mathcal{Z}\left(\hat{J}_{1} \otimes J_{2}\right) \mathcal{Z}^{*} \quad$ and $\quad \hat{J}=\mathcal{Z}\left(J_{1} \otimes \hat{J}_{2}\right) \mathcal{Z}^{*}$;

- there exist group-like unitaries $k_{1}^{i t} \in M_{1}$ and $k_{2}^{i t} \in M_{2}$ such that

$$
\mathcal{Z}^{*}\left(\hat{\delta}_{1}^{i t} \otimes 1\right) \mathcal{Z}=\hat{\delta}_{1}^{i t} \otimes k_{2}^{-i t} \quad \text { and } \quad \mathcal{Z}^{*}\left(1 \otimes \hat{\delta}_{2}^{i t}\right) \mathcal{Z}=k_{1}^{-i t} \otimes \hat{\delta}_{2}^{i t}
$$

and $\rho_{1}^{i t}=k_{1}^{i t} \delta_{1}^{i t}, \rho_{2}^{i t}=k_{2}^{i t} \delta_{2}^{i t}$ (observe that $\rho_{1}$ and $\rho_{2}$ are defined in Proposition 4.2, associated with a matching $\mathrm{m})$;

- $Z=\nu_{1}^{i / 4} \mathcal{Z}\left(J_{1} \otimes J_{2}\right) \mathcal{Z}\left(J_{1} \otimes J_{2}\right)$.

The double crossed product is unimodular if and only if $k_{1}=\delta_{1}$ and $k_{2}=\delta_{2}$. This is the case for the ordinary quantum double of a l.c. quantum group.

Proof. To determine the bicrossed product, we calculate:

$$
\begin{aligned}
\left(\mathcal{Z}^{*} \otimes \mathcal{Z}^{*}\right) W(\mathcal{Z} \otimes \mathcal{Z}) & =\mathcal{Z}_{34}^{*} W_{2,24} \mathcal{Z}_{12}^{*} \mathcal{Z}_{34} \hat{W}_{1,13} \mathcal{Z}_{12} \\
& =W_{2,24}\left(\iota \otimes \Delta_{2}\right)\left(\mathcal{Z}^{*}\right)_{324} \mathcal{Z}_{12}^{*} \mathcal{Z}_{34}\left(\Delta_{1}^{\mathrm{op}} \otimes \iota\right)(\mathcal{Z})_{132} \hat{W}_{1,13}=W_{2,24} \hat{W}_{1,13} .
\end{aligned}
$$

This proves that $\operatorname{Ad} \mathcal{Z}^{*}$ maps $(M, \Delta)$ onto the tensor product $\left(\hat{M}_{1} \otimes M_{2},(\iota \otimes \sigma \otimes \iota)\left(\hat{\Delta}_{1} \otimes \Delta_{2}\right)\right)$. From Proposition 4.2 in [13], the formulas for $J$ and $\hat{J}$ follow.

Because $\mathcal{Z}^{*}$ is a corepresentation for $\left(M_{1}, \Delta_{1}\right)$, we get that $(\iota \otimes \omega)\left(\mathcal{Z}^{*}\right) \in D\left(S_{1}\right)$ for all $\omega \in M_{2 *}$ and $S_{1}(\iota \otimes \omega)\left(\mathcal{Z}^{*}\right)=(\iota \otimes \omega)(\mathcal{Z})$. An analogous statement holds for the left slices of $\mathcal{Z}$ and $S_{2}$. Combining both, we conclude that $\mathcal{Z}$ is invariant under $\tau_{-t}^{1} \otimes \tau_{t}^{2}$ and $R_{1} \otimes R_{2}$. The formula for $Z$ then follows.

Finally, $\eta_{t}^{1}(x)=\hat{\delta}_{1}^{i t} x \hat{\delta}_{1}^{-i t}$ defines a one-parameter group of automorphisms of $M_{1}$ satisfying $\Delta_{1} \eta_{t}^{1}=\left(\eta_{t}^{1} \otimes \iota\right) \Delta_{1}$. It follows that $\left(\eta_{t}^{1} \otimes \iota\right)\left(\mathcal{Z}^{*}\right) \mathcal{Z}$ is invariant under $\Delta_{1} \otimes \iota$. Hence, there exist unitaries $k_{2}^{i t} \in M_{2}$ such that $\mathcal{Z}^{*}\left(\hat{\delta}_{1}^{i t} \otimes 1\right) \mathcal{Z}=\hat{\delta}_{1}^{i t} \otimes k_{2}^{-i t}$. Applying $\iota \otimes \Delta_{2}$ to this equality, we find that $k_{2}^{i t}$ is group-like. We analogously find $k_{1}^{i t}$. Because the modular element of the tensor product $\hat{M}_{1} \otimes M_{2}$ is given by $\hat{\delta}_{1} \otimes \delta_{2}$, we get the following formula for the modular element $\delta$ of $(M, \Delta)$ :

$$
\delta^{i t}=\mathcal{Z}\left(\hat{\delta}_{1}^{i t} \otimes \delta_{2}^{i t}\right) \mathcal{Z}^{*}=\left(\hat{\delta}_{1}^{i t} \otimes 1\right) \alpha\left(k_{2}^{i t} \delta_{2}^{i t}\right) .
$$

So, by definition, $\rho_{2}^{i t}=k_{2}^{i t} \delta_{2}^{i t}$.

It follows immediately from Theorem 5.3 that $\left(M_{\mathrm{m}}, \Delta_{\mathrm{m}}\right)$ is unimodular if and only if $k_{1}=\delta_{1}$ and $k_{2}=\delta_{2}$. This is the case for the ordinary quantum double: we have $\left(M_{1}, \Delta_{1}\right)=\left(N, \Delta_{N}^{\text {op }}\right)$ and hence, $\hat{\delta}_{1}^{i t}=\hat{J}_{N} \hat{\delta}_{N}^{-i t} \hat{J}_{N}$ satisfying

$$
\mathcal{Z}^{*}\left(\hat{\delta}_{1}^{i t} \otimes 1\right) \mathcal{Z}=\left(\hat{J}_{N} \otimes J_{N}\right) W_{N}\left(\hat{\delta}_{N}^{-i t} \otimes 1\right) W_{N}^{*}\left(\hat{J}_{N} \otimes J_{N}\right)=\hat{J}_{N} \hat{\delta}_{N}^{-i t} \hat{J}_{N} \otimes \hat{\delta}_{N}^{-i t}=\hat{\delta}_{1}^{i t} \otimes \delta_{2}^{-i t} .
$$

So, we are done.

\section{$9 \quad \mathrm{C}^{*}$-algebraic features of bicrossed and double crossed products}

Throughout this section, we fix two l.c. quantum groups $\left(M_{1}, \Delta_{1}\right),\left(M_{2}, \Delta_{2}\right)$ and a matching $\mathrm{m}$ on them. We have an associated bicrossed product $(M, \Delta)$ and double crossed product $\left(M_{\mathrm{m}}, \Delta_{\mathrm{m}}\right)$. We continue to use the notations fixed in Notation 2.4. So, the $\mathrm{C}^{*}$-algebras associated to $\left(M_{1}, \Delta_{1}\right),\left(M_{2}, \Delta_{2}\right),(M, \Delta)$ and $\left(M_{\mathrm{m}}, \Delta_{\mathrm{m}}\right)$ are denoted by $A_{1}, A_{2}, A$ and $A_{\mathrm{m}}$, respectively.

We denote by $\mathcal{K}$ the $\mathrm{C}^{*}$-algebra of compact operators on $H_{1} \otimes H_{2}$ and by $\mathcal{K}_{i}$ the $\mathrm{C}^{*}$-algebra of compact operators on $H_{i}, i=1,2$.

The following result is rather easy to prove. We give formulas for the $\mathrm{C}^{*}$-algebras $A, \hat{A}$ associated with the bicrossed product and for the $\mathrm{C}^{*}$-algebra $\hat{A}_{\mathrm{m}}$ associated with the double crossed product. Observe that it is much more delicate to describe the $\mathrm{C}^{*}$-algebra $A_{\mathrm{m}}$, see Proposition 9.5 where we give a formula in the case of regular $\left(M_{1}, \Delta_{1}\right)$ and $\left(M_{2}, \Delta_{2}\right)$. 
Proposition 9.1. We have

$$
A=\left[\left(\hat{A}_{1} \otimes 1\right) \alpha\left(A_{2}\right)\right] \quad \text { and } \quad \hat{A}=\left[\left(1 \otimes \hat{A}_{2}\right) \beta\left(A_{1}\right)\right] .
$$

Further, we have

$$
\hat{A}_{\mathrm{m}}=\left[\left(\hat{J}_{1} \hat{A}_{1} \hat{J}_{1} \otimes 1\right) Z^{*}\left(1 \otimes \hat{A}_{2}\right) Z\right] .
$$

Proof. We have

$$
\begin{aligned}
A & =\left[(\iota \otimes \iota \otimes \omega \otimes \mu)\left((\alpha \otimes \iota \otimes \iota)\left(W_{2,13} U_{\beta, 23}\right) \hat{W}_{1,13}\right)\right] \\
& =\left[(\iota \otimes \iota \otimes \omega \otimes \mu)\left((\alpha \otimes \iota \otimes \iota)\left(U_{\beta, 23} W_{2,13} U_{\beta, 21}^{*}\right) \hat{W}_{1,13}\right)\right] \\
& =\left[\alpha\left(A_{2}\right)(\iota \otimes \iota \otimes \omega)\left((\alpha \otimes \iota)\left(U_{\beta, 21}^{*}\right) \hat{W}_{1,13}\right)\right]=\left[\alpha\left(A_{2}\right)\left(\hat{A}_{1} \otimes 1\right)\right],
\end{aligned}
$$

where we used that $\left(\iota \otimes \Delta_{2}\right)\left(U_{\beta}\right)=U_{\beta, 13} U_{\beta, 12}$ and the fact that $U_{\beta} \in \mathrm{M}\left(\mathcal{K}_{1} \otimes A_{2}\right)$.

We know that $A$ is a $\mathrm{C}^{*}$-algebra. This proves the formula for $A$ and the formula for $\hat{A}$ is proven analogously. The formula for $\hat{A}_{\mathrm{m}}$ is an immediate corollary of the formula for $W_{\mathrm{m}}$ in Theorem 5.3.

Next, we present a quantum version of Theorem 3.11 in [3]: we give a necessary and sufficient condition for $(M, \Delta)$ to be (semi-)regular.

Proposition 9.2. We have that $(M, \Delta)$ is semi-regular if and only if both $\left(M_{1}, \Delta_{1}\right)$ and $\left(M_{2}, \Delta_{2}\right)$ are semi-regular and $A_{1} \otimes A_{2} \subset A_{\mathrm{m}}$.

We have that $(M, \Delta)$ is regular if and only if both $\left(M_{1}, \Delta_{1}\right)$ and $\left(M_{2}, \Delta_{2}\right)$ are regular and one of the following conditions holds true: $A_{1} \otimes A_{2}=A_{\mathrm{m}}$ or $\mathrm{m}\left(A_{1} \otimes A_{2}\right)=A_{1} \otimes A_{2}$.

Proof. First, observe that

$$
[\mathcal{C}(W)]=\left[(\iota \otimes \iota \otimes \omega \otimes \mu)\left(\Sigma_{13} \Sigma_{24} W_{2,24} U_{\beta, 34} U_{\alpha, 12}^{*} \hat{W}_{1,13}\right)\right] .
$$

Because $\left(\iota \otimes \Delta_{2}\right)\left(U_{\beta}\right)=U_{\beta, 13} U_{\beta, 12}$ and $\left(\Delta_{1} \otimes \iota\right)\left(U_{\alpha}\right)=U_{\alpha, 23} U_{\alpha, 13}$, we get

$$
[\mathcal{C}(W)]=U_{\beta}\left[(\iota \otimes \iota \otimes \omega \otimes \mu)\left(\left(\Sigma W_{2}\right)_{24}\left(U_{\beta}^{*} U_{\alpha}\right)_{12}\left(\Sigma \hat{W}_{1}\right)_{13}\right)\right] U_{\alpha}^{*}=U_{\beta}\left[\left(1 \otimes \mathcal{C}\left(W_{2}\right)\right) U_{\beta}^{*} U_{\alpha}\left(\mathcal{C}\left(\hat{W}_{1}\right) \otimes 1\right)\right] U_{\alpha}^{*} .
$$

Suppose that $(M, \Delta)$ is semi-regular. From Proposition 5.7 in [3], it follows that semi-regularity passes to closed quantum subgroups. So, $\left(M_{1}, \Delta_{1}\right)$ and $\left(M_{2}, \Delta_{2}\right)$ are semi-regular. From the calculation above, we conclude that

$$
\mathcal{K} \subset\left[\left(1 \otimes \mathcal{C}\left(W_{2}\right)\right) U_{\beta}^{*} U_{\alpha}\left(\mathcal{C}\left(\hat{W}_{1}\right) \otimes 1\right)\right]
$$

and hence,

$$
\mathcal{K}=\left[\left(1 \otimes \mathcal{K}_{2}\right) \mathcal{K}\left(\mathcal{K}_{1} \otimes 1\right)\right] \subset\left[\left(1 \otimes \mathcal{K}_{2}\right) U_{\beta}^{*} U_{\alpha}\left(\mathcal{K}_{1} \otimes 1\right)\right] .
$$

Hence, $\mathcal{K} \subset\left[\left(1 \otimes \mathcal{K}_{2}\right) Z^{*}\left(\mathcal{K}_{1} \otimes 1\right)\right]$.

On the other hand, we have

$$
\begin{aligned}
A_{\mathrm{m}} & =\left[(\iota \otimes \iota \otimes \omega \otimes \mu)\left(\left(\Sigma V_{1}^{*} \Sigma\right)_{13} Z_{34}^{*} W_{2,24}\right)\right]=\left[(\iota \otimes \iota \otimes \omega \otimes \mu)\left(\left(\Sigma V_{1}^{*} \Sigma\right)_{13}\left(\left(1 \otimes \mathcal{K}_{2}\right) Z^{*}\left(\mathcal{K}_{1} \otimes 1\right)\right)_{34} W_{2,24}\right)\right] \\
& \supset\left[(\iota \otimes \iota \otimes \omega \otimes \mu)\left(\left(\Sigma V_{1}^{*} \Sigma\right)_{13}\left(\mathcal{K}_{1} \otimes \mathcal{K}_{2}\right)_{34} W_{2,24}\right)\right]=A_{1} \otimes A_{2} .
\end{aligned}
$$

Conversely, suppose that $\left(M_{1}, \Delta_{1}\right)$ and $\left(M_{2}, \Delta_{2}\right)$ are both semi-regular and $A_{1} \otimes A_{2} \subset A_{\mathrm{m}}$. It is easy to check that $A_{1} \otimes A_{2}=\left[A_{\mathrm{m}}\left(A_{1} \otimes 1\right)\right]$. Hence,

$$
\mathrm{m}\left(A_{1} \otimes A_{2}\right)=\left[\left(R_{1} \otimes R_{2}\right) R_{\mathrm{m}}\left(A_{\mathrm{m}}\right) \beta\left(A_{1}\right)\right] \supset\left[\left(A_{1} \otimes A_{2}\right) \beta\left(A_{1}\right)\right] .
$$

But, using Proposition 9.1, we have

$$
[A \hat{A}]=\left[\left(\hat{A}_{1} \otimes 1\right) \mathrm{m}\left(A_{1} \otimes A_{2}\right)\left(1 \otimes \hat{A}_{2}\right)\right] \supset\left[\left(\hat{A}_{1} A_{1} \otimes A_{2}\right) \beta\left(A_{1}\right)\left(1 \otimes \hat{A}_{2}\right)\right] \supset\left[\left(\mathcal{K}_{1} \otimes A_{2}\right) \beta\left(A_{1}\right)\left(1 \otimes \hat{A}_{2}\right)\right] .
$$


Because $[A \hat{A}]$ is a $\mathrm{C}^{*}$-algebra, we get

$$
[A \hat{A}] \supset \mathcal{K}_{1} \otimes\left[A_{2}(\omega \otimes \iota) \beta\left(A_{1}\right) \hat{A}_{2}\right]=\mathcal{K}_{1} \otimes\left[A_{2} \hat{A}_{2}\right] \supset \mathcal{K}_{1} \otimes \mathcal{K}_{2}
$$

where we used that, for $x \in A_{1}, \beta(x)=U_{\beta}(x \otimes 1) U_{\beta}^{*}$ with $U_{\beta} \in \mathrm{M}\left(\mathcal{K}_{1} \otimes A_{2}\right)$. Hence, $(M, \Delta)$ is semi-regular. This concludes the proof of the first statement of the proposition.

In order to prove the second statement, we can replace above every inclusion by an equality to obtain the equivalence between regularity of $(M, \Delta)$ and regularity of $\left(M_{1}, \Delta_{1}\right),\left(M_{2}, \Delta_{2}\right)$ and the equality $A_{\mathrm{m}}=A_{1} \otimes A_{2}$. The only different point is to prove that the regularity of $(M, \Delta)$ implies the regularity of $\left(M_{1}, \Delta_{1}\right)$ and $\left(M_{2}, \Delta_{2}\right)$. But, suppose that $(M, \Delta)$ is regular. Then, $(\alpha \otimes \iota)\left(W_{1}\right)$ is a corepresentation of $(M, \Delta)$ and satisfies as such

$$
\left[\left(\mathcal{K}_{1} \otimes \mathcal{K}_{2} \otimes 1\right)(\alpha \otimes \iota)\left(W_{1}\right)\left(1 \otimes 1 \otimes \mathcal{K}_{1}\right)\right]=\mathcal{K}_{1} \otimes \mathcal{K}_{2} \otimes \mathcal{K}_{1}
$$

Because $\alpha(x)=U_{\alpha}(1 \otimes x) U_{\alpha}^{*}$, it follows immediately that $\left(M_{1}, \Delta_{1}\right)$ is regular. We get the regularity of $\left(M_{2}, \Delta_{2}\right)$ in an analogous way.

Finally, we suppose that $\left(M_{1}, \Delta_{1}\right)$ and $\left(M_{2}, \Delta_{2}\right)$ are regular and $\mathrm{m}\left(A_{1} \otimes A_{2}\right)=A_{1} \otimes A_{2}$. Using Proposition 9.1 , we immediately have

$$
[A \hat{A}]=\left[\left(\hat{A}_{1} \otimes 1\right) \mathrm{m}\left(A_{1} \otimes A_{2}\right)\left(1 \otimes \hat{A}_{2}\right)\right]=\left[\hat{A}_{1} A_{1} \otimes A_{2} \hat{A}_{2}\right]=\mathcal{K}_{1} \otimes \mathcal{K}_{2}
$$

yielding the regularity of $(M, \Delta)$.

The (semi-)regularity of the double crossed product $\left(M_{\mathrm{m}}, \Delta_{\mathrm{m}}\right)$ is characterized more easily in the following proposition.

Proposition 9.3. We have that $\left(M_{\mathrm{m}}, \Delta_{\mathrm{m}}\right)$ is semi-regular (resp., regular) if and only if both $\left(M_{1}, \Delta_{1}\right)$ and $\left(M_{2}, \Delta_{2}\right)$ are semi-regular (resp., regular).

Proof. First observe that

$$
\left[\mathcal{C}\left(W_{\mathrm{m}}\right)\right]=\left[(\iota \otimes \iota \otimes \omega \otimes \mu)\left(\left(\Sigma V_{1}\right)_{13}^{*} Z_{32}^{*}\left(\Sigma W_{2}\right)_{24}\right)\right]=\left[(\iota \otimes \iota \otimes \omega)\left(\left(\Sigma V_{1}\right)_{13}^{*} Z_{32}^{*}\right)\left(1 \otimes \mathcal{C}\left(W_{2}\right)\right)\right] .
$$

Suppose that $\left(M_{1}, \Delta_{1}\right)$ and $\left(M_{2}, \Delta_{2}\right)$ are semi-regular. Then, it follows that

$$
\left[\mathcal{C}\left(W_{\mathrm{m}}\right)\right] \supset\left[(\iota \otimes \iota \otimes \omega)\left(\left(\Sigma V_{1}\right)_{13}^{*} Z_{32}^{*}\right)\left(1 \otimes \mathcal{K}_{2}\right)\right]=\left[(\iota \otimes \iota \otimes \omega)\left(\left(\Sigma V_{1}\right)_{13}^{*}\right)\left(1 \otimes \mathcal{K}_{2}\right)\right] \supset \mathcal{K}_{1} \otimes \mathcal{K}_{2} .
$$

Hence, $\left(M_{\mathrm{m}}, \Delta_{\mathrm{m}}\right)$ is semi-regular.

Suppose, conversely, that $\left(M_{\mathrm{m}}, \Delta_{\mathrm{m}}\right)$ is semi-regular. Because $\left(\hat{M}_{1}^{\prime}, \hat{\Delta}_{1}^{\prime}\right)$ and $\left(\hat{M}_{2}, \hat{\Delta}_{2}\right)$ are closed quantum subgroups of $\left(\hat{M}_{\mathrm{m}}, \hat{\Delta}_{\mathrm{m}}\right)$, we get that they are semi-regular, using Proposition 5.7 in [3]. Hence, the same holds for $\left(M_{1}, \Delta_{1}\right)$ and $\left(M_{2}, \Delta_{2}\right)$.

To prove the statement about regularity, we proceed exactly as in the final paragraph of the proof of the previous proposition.

Finally, we want to give some more detailed information about the $\mathrm{C}^{*}$-algebras $A_{\mathrm{m}}$ and $A$, as well as the crossed product $A \rtimes_{\mathrm{r}} A$ of the right action of $(A, \Delta)$ on itself. Our results will be quantum versions of the following results in [3]: Proposition 3.6, Lemma 3.9 and Proposition 3.10.

To obtain our results, we need actions of $\mathrm{C}^{*}$-algebraic quantum groups on $\mathrm{C}^{*}$-algebras. As shown in Section 5 of [3], the notion of continuity of such an action is problematic when the acting quantum group is non-regular. So, for the rest of this section, we suppose that $\left(M_{1}, \Delta_{1}\right)$ and $\left(M_{2}, \Delta_{2}\right)$ are regular.

\section{Proposition 9.4. Defining}

$$
T_{1}=\left[(\iota \otimes \mu) \beta\left(A_{1}\right) \mid \mu \in \mathrm{B}\left(H_{2}\right)_{*}\right] \quad \text { and } \quad T_{2}=\left[(\omega \otimes \iota) \alpha\left(A_{2}\right) \mid \omega \in \mathrm{B}\left(H_{1}\right)_{*}\right],
$$

we have that $T_{1}$ and $T_{2}$ are $C^{*}$-algebras, such that the restrictions of $\alpha$ and $\beta$ :

$$
\alpha: T_{2} \rightarrow \mathrm{M}\left(A_{1} \otimes T_{2}\right), \quad \beta: T_{1} \rightarrow \mathrm{M}\left(T_{1} \otimes A_{2}\right)
$$


define $C^{*}$-algebraic actions of $\left(A_{1}, \Delta_{1}\right)$ on the left on $T_{2}$ and of $\left(A_{2}, \Delta_{2}^{\mathrm{op}}\right)$ on the right on $T_{1}$, which are continuous in the strong sense:

$$
\left[\alpha\left(T_{2}\right)\left(A_{1} \otimes 1\right)\right]=A_{1} \otimes T_{2} \quad \text { and } \quad\left[\beta\left(T_{1}\right)\left(1 \otimes A_{2}\right)\right]=T_{1} \otimes A_{2} .
$$

Moreover, we have

$$
A=A_{1 \mathrm{r}} \ltimes T_{2} \quad \text { and } \quad \hat{A}=T_{1} \rtimes_{\mathrm{r}} A_{2} .
$$

Proof. As in the proof of Proposition 5.7 in [3], we conclude that $T_{1}$ and $T_{2}$ are $\mathrm{C}^{*}$-algebras. Further, we observe that

$$
\begin{aligned}
{\left[\alpha\left(T_{2}\right)\left(A_{1} \otimes 1\right)\right] } & =\left[(\omega \otimes \iota \otimes \iota)\left(V_{1,12} \alpha\left(A_{2}\right)_{13} V_{1,12}^{*}\left(1 \otimes A_{1} \otimes 1\right)\right)\right] \\
& =\left[(\omega \otimes \iota \otimes \iota)\left(\left(\left(\mathcal{K}_{1} \otimes 1\right) V_{1}\left(1 \otimes A_{1}\right)\right)_{12} \alpha\left(A_{2}\right)_{13}\right)\right]=A_{1} \otimes T_{2},
\end{aligned}
$$

where we used that $V_{1} \in \mathrm{M}\left(\mathcal{K}_{1} \otimes A_{1}\right)$ and $\left[\left(\mathcal{K}_{1} \otimes 1\right) V_{1}\left(1 \otimes A_{1}\right)\right]=\mathcal{K}_{1} \otimes A_{1}$ by regularity of $\left(M_{1}, \Delta_{1}\right)$. It follows that $\alpha: T_{2} \rightarrow \mathrm{M}\left(A_{1} \otimes T_{2}\right)$ is non-degenerate and that the restriction of $\alpha$ to $T_{2}$ is continuous in the strong sense. We analogously show the same kind of properties for $T_{1}$ and $\beta$.

By Definition 7.1 in [2], we have $A_{1 \mathrm{r} \ltimes} \ltimes T_{2}=\left[\left(\hat{A}_{1} \otimes 1\right) \alpha\left(T_{2}\right)\right]$ and this a $\mathrm{C}^{*}$-algebra. So,

$$
\begin{aligned}
A_{1 \mathrm{r}} \ltimes T_{2} & =\left[\left(\hat{A}_{1} \otimes 1\right) \alpha\left(T_{2}\right)\left(\hat{A}_{1} \otimes 1\right)\right]=\left[(\omega \otimes \iota \otimes \iota)\left(\left(1 \otimes \hat{A}_{1} \otimes 1\right) W_{1,12}^{*} \alpha\left(A_{2}\right)_{23} W_{1,12}\left(1 \otimes \hat{A}_{1} \otimes 1\right)\right)\right] \\
& =\left[\left(\hat{A}_{1} \otimes 1\right) \alpha\left(A_{2}\right)\left(\hat{A}_{1} \otimes 1\right)\right],
\end{aligned}
$$

because $W_{1} \in \mathrm{M}\left(\mathcal{K}_{1} \otimes \hat{A}_{1}\right)$. But, from Proposition 9.1, we know that $A=\left[\left(\hat{A}_{1} \otimes 1\right) \alpha\left(A_{2}\right)\right]$ and we know that $A$ is a $\mathrm{C}^{*}$-algebra. Hence, we are done.

Recall that comparing our work with [3], the double crossed product $\left(M_{\mathrm{m}}, \Delta_{\mathrm{m}}\right)$ should be considered as the 'big' group $G$ in [3]. Hence, the following result is really the quantum version of [3], Lemma 3.9 and Proposition 3.10 .

Proposition 9.5. We have

$$
A_{\mathrm{m}}=\left[(\iota \otimes \iota \otimes \omega)\left(\mathrm{m}_{13}\left(\iota \otimes \Delta_{2}^{\mathrm{op}}\right)\left(A_{1} \otimes A_{2}\right)\right) \mid \omega \in \mathrm{B}\left(H_{2}\right)_{*}\right] .
$$

Further,

$$
\Delta_{1}^{\mathrm{op}} \otimes \Delta_{2}: A_{\mathrm{m}} \rightarrow \mathrm{M}\left(A_{1} \otimes A_{\mathrm{m}} \otimes A_{2}\right)
$$

defines a $C^{*}$-algebraic action of $\left(A_{1}, \Delta_{1}^{\mathrm{op}}\right)$ on the left and of $\left(A_{2}, \Delta_{2}\right)$ on the right on $A_{\mathrm{m}}$. This action is continuous in the strong sense:

$$
\left[\left(A_{1} \otimes 1 \otimes 1 \otimes 1\right)\left(\Delta_{1}^{\mathrm{op}} \otimes \Delta_{2}\right)\left(A_{\mathrm{m}}\right)\left(1 \otimes 1 \otimes 1 \otimes A_{2}\right)\right]=A_{1} \otimes A_{\mathrm{m}} \otimes A_{2} .
$$

Moreover, the crossed product satisfies:

$$
A \rtimes_{\mathrm{r}} A \cong A_{1 \mathrm{r}} \ltimes A_{\mathrm{m}} \rtimes_{\mathrm{r}} A_{2} .
$$

Proof. One checks easily that $A_{1} \otimes A_{2}=\left[A_{\mathrm{m}}\left(A_{1} \otimes 1\right)\right]$. Hence, it follows that

$$
\begin{aligned}
{\left[(\iota \otimes \iota \otimes \omega)\left(\mathrm{m}_{13}\left(\iota \otimes \Delta_{2}^{\mathrm{op}}\right)\left(A_{1} \otimes A_{2}\right)\right)\right] } & =\left[(\iota \otimes \iota \otimes \omega)\left(\mathrm{m}_{13}\left(\iota \otimes \Delta_{2}^{\mathrm{op}}\right)\left(A_{\mathrm{m}}\right) \mathrm{m}\left(A_{1} \otimes 1\right)_{13}\right)\right] \\
& =\left[(\iota \otimes \iota \otimes \omega)\left(\mathrm{m}_{12}^{-1}\left(\left(1 \otimes 1 \otimes A_{2}\right)\left(\iota \otimes \Delta_{2}^{\mathrm{op}}\right) \mathrm{m}\left(A_{\mathrm{m}}\right)\right) \mathrm{m}\left(A_{1} \otimes 1\right)_{13}\right)\right] .
\end{aligned}
$$

From Proposition 3.5, we get that

$\left[\left(1 \otimes 1 \otimes A_{2}\right)\left(\iota \otimes \Delta_{2}^{\mathrm{op}}\right) \mathrm{m}\left(A_{\mathrm{m}}\right)\right]=\left[(\iota \otimes \iota \otimes \iota \otimes \omega \otimes \mu)\left(\left(\left(A_{2} \otimes 1\right) W_{2}\right)_{35} W_{2,25} Z_{45}\left(\Sigma V_{1}^{*} \Sigma\right)_{14}\right)\right]=\mathrm{m}\left(A_{\mathrm{m}}\right) \otimes A_{2}$, because $W_{2} \in \mathrm{M}\left(A_{2} \otimes \mathcal{K}_{2}\right)$. Hence, we conclude that

$$
\left[(\iota \otimes \iota \otimes \omega)\left(\mathrm{m}_{13}\left(\iota \otimes \Delta_{2}^{\mathrm{op}}\right)\left(A_{1} \otimes A_{2}\right)\right)\right]=\left[A_{\mathrm{m}}\left(T_{1} \otimes 1\right)\right] .
$$


Now, we use the second formula for $V_{\mathrm{m}}$ in Theorem 5.3 and we find that

$$
\left[\left(T_{1} \otimes 1\right) A_{\mathrm{m}}\right]=\left[(\omega \otimes \iota \otimes \iota)\left(\left(\left(T_{1} \otimes \mathcal{K}_{2}\right) \beta\left(A_{1}\right)\right)_{21} U_{\alpha, 21} V_{2,13}\right)\right] .
$$

Next, we claim that $\left[\left(T_{1} \otimes \mathcal{K}_{2}\right) \beta\left(A_{1}\right)\right]=\left[\left(1 \otimes \mathcal{K}_{2}\right) \beta\left(A_{1}\right)\right]$. Because $\beta$ is continuous in the strong sense on $T_{1}$, we have $\left[\left(1 \otimes \mathcal{K}_{2}\right) \beta\left(T_{1}\right)\right]=T_{1} \otimes \mathcal{K}_{2}$. To prove our claim, it suffices to show that $\left[T_{1} A_{1}\right]=A_{1}$. This last statement can be proven as follows: from Theorem 5.3, we know that $(\iota \otimes \mathrm{m})\left(U_{\beta, 13} V_{1,12}\right)=V_{1,12} U_{\beta, 13}$, which gives

$$
T_{1}=\left[(\omega \otimes \iota \otimes \mu)\left((\iota \otimes \mathrm{m})\left(U_{\beta, 13}^{*}\right) V_{1,12}\right)\right]
$$

and so, because $V_{1} \in \mathrm{M}\left(\mathcal{K}_{1} \otimes A_{1}\right)$ and $U_{\alpha} \in \mathrm{M}\left(A_{1} \otimes \mathcal{K}_{2}\right)$,

$$
\left[T_{1} A_{1}\right]=\left[(\omega \otimes \iota \otimes \mu)(\iota \otimes \alpha)\left(U_{\beta}^{*}\right) A_{1}\right]=\left[(\omega \otimes \iota \otimes \mu)\left(U_{\alpha, 23} U_{\beta, 13}^{*} U_{\alpha, 23}^{*}\right) A_{1}\right]=A_{1} .
$$

Hence, our claim is proven. Combining this with Equation (9.2), we get

$$
\left[\left(T_{1} \otimes 1\right) A_{\mathrm{m}}\right]=\left[(\omega \otimes \iota \otimes \iota)\left(\left(\left(1 \otimes \mathcal{K}_{2}\right) \beta\left(A_{1}\right)\right)_{21} U_{\alpha, 21} V_{2,13}\right)\right]=A_{\mathrm{m}},
$$

where we used again the second formula for $V_{\mathrm{m}}$ in Theorem 5.3. Combining this equation with Equation (9.1), we finally arrive at

$$
\left[(\iota \otimes \iota \otimes \omega)\left(\mathrm{m}_{13}\left(\iota \otimes \Delta_{2}^{\mathrm{op}}\right)\left(A_{1} \otimes A_{2}\right)\right)\right]=A_{\mathrm{m}} .
$$

It is easy to check that $\Delta_{1}^{\mathrm{op}} \otimes \Delta_{2}$ defines a $\mathrm{C}^{*}$-algebraic action of $\left(A_{1}, \Delta_{1}^{\mathrm{op}}\right)$ on the left and $\left(A_{2}, \Delta_{2}\right)$ on the right on $A_{\mathrm{m}}$, which is continuous in the strong sense.

Because $R_{\mathrm{m}}\left(A_{\mathrm{m}}\right)=A_{\mathrm{m}}$, we also get

$$
A_{\mathrm{m}}=\left[(\iota \otimes \iota \otimes \omega)\left(\iota \otimes \Delta_{2}\right) \mathrm{m}^{-1}\left(A_{1} \otimes A_{2}\right)\right] .
$$

Now, by Definition 7.1 in [2], $A \rtimes_{\mathrm{r}} A=[A \hat{J} \hat{A} \hat{J}]$. Because $\hat{J} A \hat{J}=A$, we get, using $\operatorname{Ad}\left(\left(\hat{J}_{1} \otimes \hat{J}_{2}\right) \hat{J}\right)$,

$$
A \rtimes_{\mathrm{r}} A \cong\left(\hat{J}_{1} \otimes \hat{J}_{2}\right)[A \hat{A}]\left(\hat{J}_{1} \otimes \hat{J}_{2}\right) .
$$

On the other hand, by definition,

$$
A_{1 \mathrm{r} \ltimes} A_{\mathrm{m}} \rtimes_{\mathrm{r}} A_{2}=\left[\left(\hat{J}_{1} \hat{A}_{1} \hat{J}_{1} \otimes 1 \otimes 1 \otimes \hat{J}_{2} \hat{A}_{2} \hat{J}_{2}\right)\left(\Delta_{1}^{\mathrm{op}} \otimes \Delta_{2}\right)\left(A_{\mathrm{m}}\right)\right],
$$

which, by using the unitary $\hat{W}_{1}^{*} \otimes W_{2}$, is isomorphic with $\left[\left(\hat{J}_{1} \hat{A}_{1} \hat{J}_{1} \otimes \hat{J}_{2} \hat{A}_{2} \hat{J}_{2}\right) A_{\mathrm{m}}\right]$.

Now, we observe that

$$
\left(\hat{J}_{1} \otimes \hat{J}_{2}\right)[A \hat{A}]\left(\hat{J}_{1} \otimes \hat{J}_{2}\right)=\left[\left(\hat{J}_{1} \hat{A}_{1} \hat{J}_{1} \otimes 1\right) \mathrm{m}^{-1}\left(A_{1} \otimes A_{2}\right)\left(1 \otimes \hat{J}_{2} \hat{A}_{2} \hat{J}_{2}\right)\right] .
$$

Because the left hand side is a $\mathrm{C}^{*}$-algebra, we have

$$
\begin{aligned}
\left(\hat{J}_{1} \otimes \hat{J}_{2}\right)[A \hat{A}]\left(\hat{J}_{1} \otimes \hat{J}_{2}\right) & =\left[\left(\hat{J}_{1} \hat{A}_{1} \hat{J}_{1} \otimes \hat{J}_{2} \hat{A}_{2} \hat{J}_{2}\right) \mathrm{m}^{-1}\left(A_{1} \otimes A_{2}\right)\left(1 \otimes \hat{J}_{2} \hat{A}_{2} \hat{J}_{2}\right)\right] \\
& =\left[\left(\hat{J}_{1} \hat{A}_{1} \hat{J}_{1} \otimes \hat{J}_{2} \hat{A}_{2} \hat{J}_{2}\right)(\iota \otimes \iota \otimes \omega)\left(\mathrm{m}^{-1}\left(A_{1} \otimes A_{2}\right)_{12} V_{2,23}^{*}\right)\right] \\
& =\left[\left(\hat{J}_{1} \hat{A}_{1} \hat{J}_{1} \otimes \hat{J}_{2} \hat{A}_{2} \hat{J}_{2}\right)(\iota \otimes \iota \otimes \omega)\left(V_{2,23}^{*}\left(\iota \otimes \Delta_{2}\right) \mathrm{m}^{-1}\left(A_{1} \otimes A_{2}\right)\right)\right] \\
& =\left[\left(\hat{J}_{1} \hat{A}_{1} \hat{J}_{1} \otimes \hat{J}_{2} \hat{A}_{2} \hat{J}_{2}\right)(\iota \otimes \iota \otimes \omega)\left(\iota \otimes \Delta_{2}\right) \mathrm{m}^{-1}\left(A_{1} \otimes A_{2}\right)\right] \\
& =\left[\left(\hat{J}_{1} \hat{A}_{1} \hat{J}_{1} \otimes \hat{J}_{2} \hat{A}_{2} \hat{J}_{2}\right) A_{\mathrm{m}}\right],
\end{aligned}
$$

where we used twice that $V_{2} \in \mathrm{M}\left(\hat{J}_{2} \hat{A}_{2} \hat{J}_{2} \otimes \mathcal{K}_{2}\right)$. We have seen above that this last subspace is isomorphic with $A_{1 \mathrm{r}} \ltimes A_{\mathrm{m}} \rtimes_{\mathrm{r}} A_{2}$. So, we are done. 


\section{Appendix: the Radon-Nikodym derivative of a weight under a quantum group action}

In this appendix, we generalize results of T. Yamanouchi $[21,22]$ and we give considerably simpler proofs.

Let $N$ be a von Neumann algebra with n.s.f. weight $\theta$. If a l.c. group $G$ acts continuously by automorphisms $\left(\alpha_{g}\right)$ on $N$, we can consider the Connes cocycles (Radon-Nikodym derivatives) $\left[D \theta \circ \alpha_{g}: D \theta\right]_{t}$ as a function $\mathbb{R} \rightarrow L^{\infty}(G) \otimes N$. In the case of an action of a l.c. quantum group $(M, \Delta)$ on $N$, Yamanouchi [21] defined the analogous Radon-Nikodym derivative $[D \theta \circ \alpha: D \theta]_{t} \in M \otimes N$. We repeat his definition.

We first introduce some needed preliminaries.

Fix an action $\alpha: N \rightarrow M \otimes N$ of the l.c. quantum group $(M, \Delta)$ on the von Neumann algebra $N$ and fix an n.s.f. weight $\theta$ on $N$. Denote by $\tilde{J}_{\theta}$ and $\tilde{\nabla}_{\theta}$ the modular operators of the dual weight $\tilde{\theta}$, acting on the Hilbert space $H \otimes H_{\theta}$, with respect to the canonical GNS-construction for $\tilde{\theta}$ explained in Section 2.3.

On the crossed product $M_{\alpha} \ltimes N$, we have a dual action of $\left(\hat{M}, \hat{\Delta}^{\mathrm{op}}\right)$ and the second crossed product $\hat{M}_{\hat{\alpha}} \ltimes\left(M_{\alpha} \ltimes N\right)$ is canonically isomorphic with $\mathrm{B}(H) \otimes N$ through the isomorphism $\Phi: \mathrm{B}(H) \otimes N \rightarrow$ $\hat{M}_{\hat{\alpha}} \ltimes\left(M_{\alpha} \ltimes N\right)$, see [13], Theorem 2.6. So, we can consider the bidual weight $\tilde{\tilde{\theta}}$ on $\mathrm{B}(H) \otimes N$, with canonical GNS-map

$$
(x y \otimes 1) \alpha(z) \mapsto \Lambda^{\prime}(x) \otimes \hat{\Lambda}(y) \otimes \Lambda_{\theta}(z) \quad \text { for } \quad x \in \mathcal{N}_{\varphi^{\prime}}, y \in \mathcal{N}_{\hat{\varphi}}, z \in \mathcal{N}_{\theta}
$$

and where $\Lambda^{\prime}(x)=J \Lambda(J x J)$ is the canonical GNS-map for the left invariant weight on the commutant quantum group $\left(M^{\prime}, \Delta^{\prime}\right)$, which is the dual of $\left(\hat{M}, \hat{\Delta}^{\mathrm{op}}\right)$. In the same sense as above, these elements $(x y \otimes 1) \alpha(z)$ span a core for the GNS-map of $\tilde{\tilde{\theta}}$.

From the results of [16], Section 2, it then follows that

$$
\tilde{\tilde{\theta}}\left(\alpha\left(a^{*}\right)\left(x^{*} y \otimes 1\right) \alpha(b)\right)=\operatorname{Tr}_{\hat{\nabla}}\left(x^{*} y\right) \theta\left(a^{*} b\right)
$$

for all $x, y \in \mathcal{N}_{\hat{\nabla}}, a, b \in \mathcal{N}_{\theta}$, where we use the short-hand notation $\mathcal{N}_{\hat{\nabla}}:=\mathcal{N}_{\operatorname{Tr}_{\hat{\nabla}}}$ and where $\operatorname{Tr}_{\hat{\nabla}}$ is the n.s.f. weight on $\mathrm{B}(H)$ with density $\hat{\nabla}$. Also, the elements $(y \otimes 1) \alpha(b)$ span a core for the GNS-map of $\tilde{\tilde{\theta}}$.

On $\mathrm{B}(H) \otimes N$, we have two natural weights: $\tilde{\tilde{\theta}}$ and $\operatorname{Tr}_{\hat{\nabla}} \otimes \theta$. The following result was first proven by Yamanouchi in [21] and called the Takesaki duality theorem for weights. We indicate how it follows from [13].

Lemma 10.1. The following formula holds:

$$
\left[D \tilde{\tilde{\theta}}: D\left(\operatorname{Tr}_{\hat{\nabla}} \otimes \theta\right)\right]_{t}=\tilde{\nabla}_{\theta}^{i t}\left(\hat{\nabla}^{-i t} \otimes \nabla_{\theta}^{-i t}\right) .
$$

Proof. Writing $T_{\hat{\hat{\alpha}}}$ for the operator valued weight from $\mathrm{B}(H) \otimes N$ to $M_{\alpha} \ltimes N$ associated with the integrable action $\hat{\hat{\alpha}}$, we know from [13], Proposition 5.7 that $T_{\hat{\hat{\alpha}}}(z)=\tilde{J}_{\theta} T_{\hat{\alpha}}^{-1}\left(\tilde{J}_{\theta} z \tilde{J}_{\theta}\right) \tilde{J}_{\theta}$. Here, $T_{\hat{\alpha}}$ is the operator valued weight from $M_{\alpha} \ltimes N$ to $\alpha(N)$ and $T_{\hat{\alpha}}^{-1}$ is the commutant operator valued weight from $\alpha(N)^{\prime}$ to $\left(M_{\alpha} \ltimes N\right)^{\prime}$. Using the language of spatial derivatives, this means that

$$
\left(\frac{d \tilde{\tilde{\theta}}}{d\left(1 \otimes \theta\left(J_{\theta} \cdot J_{\theta}\right)\right)}\right)^{i t}=\left(\frac{d\left(\tilde{\theta}\left(\tilde{J}_{\theta} \cdot \tilde{J}_{\theta}\right) \circ T_{\hat{\alpha}}^{-1}\right)}{d\left(\theta \circ \alpha^{-1}\right)}\right)^{-i t}=\left(\frac{d\left(\tilde{\theta}\left(\tilde{J}_{\theta} \cdot \tilde{J}_{\theta}\right)\right)}{d\left(\theta \circ \alpha^{-1} \circ T_{\hat{\alpha}}\right)}\right)^{-i t}=\left(\frac{d\left(\tilde{\theta}\left(\tilde{J}_{\theta} \cdot \tilde{J}_{\theta}\right)\right)}{d \tilde{\theta}}\right)^{-i t}=\tilde{\nabla}_{\theta}^{i t} .
$$

Then, we get that

$$
\left[D \tilde{\tilde{\theta}}: D\left(\operatorname{Tr}_{\hat{\nabla}} \otimes \theta\right)\right]_{t}=\left(\frac{d \tilde{\tilde{\theta}}}{d\left(1 \otimes \theta\left(J_{\theta} \cdot J_{\theta}\right)\right)}\right)^{i t}\left(\frac{d\left(\operatorname{Tr}_{\hat{\nabla}} \otimes \theta\right)}{d\left(1 \otimes \theta\left(J_{\theta} \cdot J_{\theta}\right)\right)}\right)^{-i t}=\tilde{\nabla}_{\theta}^{i t}\left(\hat{\nabla}^{-i t} \otimes \nabla_{\theta}^{-i t}\right) .
$$

This concludes the proof.

Definition 10.2. Define $[D \theta \circ \alpha: D \theta]_{t}:=\tilde{\nabla}_{\theta}^{i t}\left(\hat{\nabla}^{-i t} \otimes \nabla_{\theta}^{-i t}\right)$. We call $[D \theta \circ \alpha: D \theta]_{t}$ the Radon-Nikodym derivative of $\theta$ under the action $\alpha$ of $(M, \Delta)$. 
We now prove the main properties of $[D \theta \circ \alpha: D \theta]_{t}$.

Theorem 10.3. Write $D_{t}:=[D \theta \circ \alpha: D \theta]_{t}$.

- $D_{t} \in M \otimes N$.

- $(\Delta \otimes \iota)\left(D_{t}\right)=(\iota \otimes \alpha)\left(D_{t}\right)\left(1 \otimes D_{t}\right)$.

Proof. Denote by $(\tilde{\tilde{\sigma}})_{t}$ the modular automorphism group of the bidual weight $\tilde{\tilde{\theta}}$ on $\mathrm{B}(H) \otimes N$. Because the dual weight $\tilde{\theta}$ is $\hat{\delta}$-invariant (see [13], Proposition 2.5), we conclude from the proof of [13], Proposition 4.3, that $\tilde{\tilde{\sigma}}_{t}(x \otimes 1)=\hat{\delta}^{-i t} \nabla^{-i t} x \nabla^{i t} \hat{\delta}^{i t} \otimes 1$ for all $x \in M^{\prime}$. From [16], Proposition 2.4, we know that $\hat{\delta}^{-i t} \nabla^{-i t}=\hat{\nabla}^{i t} \delta^{i t}$ and hence, $\tilde{\tilde{\sigma}}_{t}(x \otimes 1)=\hat{\nabla}^{i t} x \hat{\nabla}^{-i t} \otimes 1$ for all $x \in M^{\prime}$. We easily conclude that $D_{t} \in M \otimes N$.

Next, we introduce the notation $\theta^{\alpha}:=\tilde{\tilde{\theta}}$ to stress that we take a bidual weight with respect to the action $\alpha$. Consider the action $\beta:=(\sigma \otimes \iota)(\iota \otimes \alpha)$ of $(M, \Delta)$ on $\mathrm{B}(H) \otimes N$. Because $\theta^{\alpha}$ is an n.s.f. weight on $\mathrm{B}(H) \otimes N$, we can define its bidual weight $\left(\theta^{\alpha}\right)^{\beta}$ on $\mathrm{B}(H \otimes H) \otimes N$. From the discussion above, we know a canonical GNS-map for $\left(\theta^{\alpha}\right)^{\beta}$ given by

$$
(y \otimes z \otimes 1) \beta(\alpha(x)) \mapsto \Lambda_{\hat{\nabla}}(y) \otimes \Lambda_{\hat{\nabla}}(z) \otimes \Lambda_{\theta}(x) \quad \text { for all } \quad y, z \in \mathcal{N}_{\hat{\nabla}}, x \in \mathcal{N}_{\theta},
$$

where $\Lambda_{\hat{\nabla}}$ is a GNS-map for $\operatorname{Tr}_{\hat{\nabla}}$. We also know that the elements $(y \otimes z \otimes 1) \beta(\alpha(x))$ span a core for the corresponding GNS-map. Observe that $\left(\Sigma V^{*} \Sigma\right)(\hat{\nabla} \otimes \hat{\nabla})(\Sigma V \Sigma)=Q \otimes \hat{\nabla}$, where $Q$ is the closure of $\delta \hat{\nabla}$. Also observe that $\beta(\alpha(x))=\left(\Delta^{\mathrm{op}} \otimes \iota\right) \alpha(x)$ for all $x \in N$. Using the above core for $\left(\theta^{\alpha}\right)^{\beta}$, we conclude that

$$
\left(\theta^{\alpha}\right)^{\beta} \circ \operatorname{Ad}(\Sigma V \Sigma \otimes 1)=\operatorname{Tr}_{Q} \otimes \theta^{\alpha} \quad \text { and } \quad\left(\operatorname{Tr}_{\hat{\nabla}} \otimes \operatorname{Tr}_{\hat{\nabla}} \otimes \theta\right) \circ \operatorname{Ad}(\Sigma V \Sigma \otimes 1)=\operatorname{Tr}_{Q} \otimes \operatorname{Tr}_{\hat{\nabla}} \otimes \theta .
$$

So, on the one hand, we have,

$\left[D\left(\left(\theta^{\alpha}\right)^{\beta}\right): D\left(\operatorname{Tr}_{\hat{\nabla}} \otimes \operatorname{Tr}_{\hat{\nabla}} \otimes \theta\right)\right]_{t}=(\Sigma V \Sigma \otimes 1)\left[D\left(\operatorname{Tr}_{Q} \otimes \theta^{\alpha}\right): D\left(\operatorname{Tr}_{Q} \otimes \operatorname{Tr}_{\hat{\nabla}} \otimes \theta\right)\right]_{t}\left(\Sigma V^{*} \Sigma \otimes 1\right)=\left(\Delta^{\mathrm{op}} \otimes \iota\right)\left(D_{t}\right)$.

On the other hand, we have

$$
\begin{aligned}
{\left[D\left(\left(\theta^{\alpha}\right)^{\beta}\right):\right.} & \left.D\left(\operatorname{Tr}_{\hat{\nabla}} \otimes \operatorname{Tr}_{\hat{\nabla}} \otimes \theta\right)\right]_{t}=\left[D\left(\left(\theta^{\alpha}\right)^{\beta}\right): D\left(\left(\operatorname{Tr}_{\hat{\nabla}} \otimes \theta\right)^{\beta}\right)\right]_{t}\left[D\left(\left(\operatorname{Tr}_{\hat{\nabla}} \otimes \theta\right)^{\beta}\right): D\left(\operatorname{Tr}_{\hat{\nabla}} \otimes \operatorname{Tr}_{\hat{\nabla}} \otimes \theta\right)\right]_{t} \\
& =\beta\left(\left[D \theta^{\alpha}: D\left(\operatorname{Tr}_{\hat{\nabla}} \otimes \theta\right)\right]_{t}\right)\left[D\left(\left(\operatorname{Tr}_{\hat{\nabla}} \otimes \theta^{\alpha}\right)(\sigma \otimes \iota)\right): D\left(\operatorname{Tr}_{\hat{\nabla}} \otimes \operatorname{Tr}_{\hat{\nabla}} \otimes \theta\right)\right]_{t} \\
& =(\sigma \otimes \iota)\left((\iota \otimes \alpha)\left(D_{t}\right)\left(1 \otimes D_{t}\right)\right),
\end{aligned}
$$

where we used the following well known fact from the theory of operator valued weights: $[D \tilde{\theta}: D \tilde{\mu}]_{t}=$ $\beta\left([D \theta: D \mu]_{t}\right)$ where $\beta$ is an action of a l.c. quantum group and $\theta, \mu$ are n.s.f. weights with dual weights $\tilde{\theta}, \tilde{\mu}$. Combining both calculations above, the proof is finished.

The proof of the previous theorem can be adapted to prove the following useful and seemingly obvious formula.

Lemma 10.4. Suppose that $N$ is a von Neumann algebra with n.s.f. weight $\theta$ and let $\alpha$ and $\beta$ be a actions of $\left(M_{1}, \Delta_{1}\right)$, resp. $\left(M_{2}, \Delta_{2}\right)$ on $N$. Suppose that there exists $a^{*}$-isomorphism $\mathrm{m}: M_{1} \otimes M_{2} \rightarrow M_{1} \otimes M_{2}$ ruling, in a sense, the commutation between $\alpha$ and $\beta$. More precisely,

$$
(\iota \otimes \beta) \alpha=(\mathrm{m} \sigma \otimes \iota)(\iota \otimes \alpha) \beta .
$$

If $\mathrm{m}\left(\tau_{t}^{1} \otimes \tau_{t}^{2}\right)=\left(\tau_{t}^{1} \otimes \tau_{t}^{2}\right) \mathrm{m}$, then

$$
(\iota \otimes \beta)\left([D \theta \circ \alpha: D \theta]_{t}\right)\left(1 \otimes[D \theta \circ \beta: D \theta]_{t}\right)=(\mathrm{m} \sigma \otimes \iota)\left((\iota \otimes \alpha)\left([D \theta \circ \beta: D \theta]_{t}\right)\left(1 \otimes[D \theta \circ \alpha: D \theta]_{t}\right)\right) .
$$


Proof. Consider the amplified action $\beta_{a}:=(\sigma \otimes \iota)(\iota \otimes \beta)$ of $\left(M_{2}, \Delta_{2}\right)$ on $\mathrm{B}\left(H_{1}\right) \otimes N$. First, we have the bidual weight $\theta^{\alpha}$ on $\mathrm{B}\left(H_{1}\right) \otimes N$, which has again a bidual weight $\left(\theta^{\alpha}\right)^{\beta_{a}}$ on $\mathrm{B}\left(H_{2} \otimes H_{1}\right) \otimes N$. The same calculation as in Equation (10.1) yields that

$$
\left[D\left(\theta^{\alpha}\right)^{\beta_{a}}: D\left(\operatorname{Tr}_{\hat{\nabla}_{2}} \otimes \operatorname{Tr}_{\hat{\nabla}_{1}} \otimes \theta\right)\right]_{t}=(\sigma \otimes \iota)\left((\iota \otimes \beta)\left([D \theta \circ \alpha: D \theta]_{t}\right)\left(1 \otimes[D \theta \circ \beta: D \theta]_{t}\right)\right) .
$$

By symmetry, we have an amplified action $\alpha_{a}$ and

$$
\left[D\left(\theta^{\beta}\right)^{\alpha_{a}}: D\left(\operatorname{Tr}_{\hat{\nabla}_{1}} \otimes \operatorname{Tr}_{\hat{\nabla}_{2}} \otimes \theta\right)\right]_{t}=(\sigma \otimes \iota)\left((\iota \otimes \alpha)\left([D \theta \circ \beta: D \theta]_{t}\right)\left(1 \otimes[D \theta \circ \alpha: D \theta]_{t}\right)\right) .
$$

Consider the unbounded operator $K:=J_{1} \delta_{1} J_{1} \otimes J_{2} \delta_{2} J_{2}$, affiliated with $M_{1}^{\prime} \otimes M_{2}^{\prime}$. Then, $K \otimes 1$ is invariant under the modular automorphism group of $\operatorname{Tr}_{\hat{\nabla}_{1}} \otimes \operatorname{Tr}_{\hat{\nabla}_{2}} \otimes \theta$ and it commutes with

$$
\left[D\left(\theta^{\beta}\right)^{\alpha_{a}}: D\left(\operatorname{Tr}_{\hat{\nabla}_{1}} \otimes \operatorname{Tr}_{\hat{\nabla}_{2}} \otimes \theta\right)\right]_{t} \in M_{1} \otimes M_{2} \otimes N
$$

Hence, $K \otimes 1$ is invariant under the modular automorphism group of $\left(\theta^{\beta}\right)^{\alpha_{a}}$. So, we can define the n.s.f. weight $\mu:=\left(\left(\theta^{\beta}\right)^{\alpha_{a}}\right)_{K \otimes 1}$. We know a GNS-map for $\left(\theta^{\beta}\right)^{\alpha_{a}}$ and because the closure of $\left(\hat{\nabla}_{1} \otimes \hat{\nabla}_{2}\right) K$ is $P_{1} \otimes P_{2}$, we have a canonical GNS-map for $\mu$ given by

$$
(z \otimes 1) \alpha_{a}(\beta(x)) \mapsto \Lambda_{P_{1} \otimes P_{2}}(z) \otimes \Lambda_{\theta}(x) \quad \text { for all } \quad z \in \mathcal{N}_{P_{1} \otimes P_{2}}, x \in \mathcal{N}_{\theta}
$$

The elements $(z \otimes 1) \alpha_{a}(\beta(x))$ span a core for this GNS-map. Denote by $\mathcal{Z}$ the canonical implementation of the automorphism $\mathrm{m}$, which is a unitary on $H_{1} \otimes H_{2}$. Because $\mathrm{m}$ commutes with $\tau_{t}^{1} \otimes \tau_{t}^{2}$ and because $P_{1}^{i t} \otimes P_{2}^{i t}$ is the canonical implementation of $\tau_{t}^{1} \otimes \tau_{t}^{2}$, we get that $\mathcal{Z}$ commutes with $P_{1} \otimes P_{2}$. Using the core for the GNS-map of $\left(\theta^{\beta}\right)^{\alpha_{a}}$ and using the given formula

$$
(\iota \otimes \beta) \alpha=(\operatorname{m} \sigma \otimes \iota)(\iota \otimes \alpha) \beta,
$$

we conclude that

$$
\mu=\rho \circ \operatorname{Ad}(\Sigma \mathcal{Z} \otimes 1)
$$

where $\rho:=\left(\left(\theta^{\alpha}\right)^{\beta_{a}}\right)_{\Sigma K \Sigma \otimes 1}$.

Finally, it follows from Equation (10.3) that

$$
\left[D \mu: D\left(\operatorname{Tr}_{P_{1} \otimes P_{2}} \otimes \theta\right)\right]_{t}=(\sigma \otimes \iota)\left((\iota \otimes \alpha)\left([D \theta \circ \beta: D \theta]_{t}\right)\left(1 \otimes[D \theta \circ \alpha: D \theta]_{t}\right)\right),
$$

while it follows from Equation (10.2) that

$$
\left[D \rho: D\left(\operatorname{Tr}_{P_{2} \otimes P_{1}} \otimes \theta\right)\right]_{t}=(\sigma \otimes \iota)\left((\iota \otimes \beta)\left([D \theta \circ \alpha: D \theta]_{t}\right)\left(1 \otimes[D \theta \circ \beta: D \theta]_{t}\right)\right) .
$$

Applying $\operatorname{Ad}(\Sigma \mathcal{Z} \otimes 1)$ and using that $\mu=\rho \circ \operatorname{Ad}(\Sigma \mathcal{Z} \otimes 1)$ and that $\mathcal{Z}$ commutes with $P_{1} \otimes P_{2}$, we arrive at the statement of the lemma.

As a corollary, we prove the following result on closed quantum subgroups.

Proposition 10.5. Let $(M, \Delta)$ be a l.c. quantum group. Suppose that $N \subset M$ is a von Neumann subalgebra such that $\Delta(N) \subset N \otimes N$

Then, $\left(N,\left.\Delta\right|_{N}\right)$ is a l.c. quantum group if and only if $R(N)=N$ and $\tau_{t}(N)=N$ for all $t \in \mathbb{R}$.

Proof. If $\left(N,\left.\Delta\right|_{N}\right)$ is a l.c. quantum group, the inclusion map is a morphism and we know that $R(N)=N$ and $\tau_{t}(N)=N$ for all $t \in \mathbb{R}$ by Proposition 5.45 in [8]. So, we only have to prove the converse implication.

Define $\alpha:=\left.\Delta\right|_{N}$, which we consider as a left action of $(M, \Delta)$ on $N$ and $\beta:=\left.\Delta^{\mathrm{op}}\right|_{N}$, which we consider as a left action of $\left(M, \Delta^{\mathrm{op}}\right)$ on $N$. Choose an n.s.f. weight $\theta$ on $N$ and define

$$
u_{t}:=[D \theta \circ \alpha: D \theta]_{t} \in M \otimes N, \quad w_{t}:=[D \theta \circ \beta: D \theta]_{t} \in M \otimes N .
$$


We will show that there exists a $\left(\sigma_{t}^{\theta}\right)$-cocycle $\left(v_{t}\right)$ in $N$ such that $u_{t}=\alpha\left(v_{t}^{*}\right)\left(1 \otimes v_{t}\right)$ for all $t \in \mathbb{R}$. If we then correct the weight $\theta$ with the cocycle $\left(v_{t}\right)$, we find a left invariant weight on $\left(N,\left.\Delta\right|_{N}\right)$.

Because $\alpha(N) \subset N \otimes N$, we can use the argument of the proof of Proposition 3.12 in [13] to conclude that $u_{t} \in N \otimes N$ and analogously, $w_{t} \in N \otimes N$ for all $t \in \mathbb{R}$. Remark that at this point, we use in a crucial way that $R$ and $\tau_{t}$ leave $N$ globally invariant.

By Theorem 10.3, we know that $(\Delta \otimes \iota)\left(u_{t}\right)=(\iota \otimes \Delta)\left(u_{t}\right)\left(1 \otimes u_{t}\right)$, which we can rewrite as

$$
(\Delta \otimes \iota)\left(\tilde{u}_{t} W\right)=\left(\tilde{u}_{t} W\right)_{13}\left(\tilde{u}_{t} W\right)_{23} \quad \text { with } \quad \tilde{u}_{t}=(\hat{J} \otimes J) u_{t}^{*}(\hat{J} \otimes J)
$$

Because $\tilde{u}_{t} \in M \otimes M^{\prime}$, the corepresentation $\tilde{u}_{t} W$ is covariant with respect to the standard representation of $M$ on $H$ in the sense that $\Delta(x)=\left(\tilde{u}_{t} W\right)^{*}(1 \otimes x)\left(\tilde{u}_{t} W\right)$ for all $x \in M$. As we remarked after Definition 2.4 in [3], this gives the existence of faithful, normal ${ }^{*}$-homomorphisms $\hat{\pi}_{t}: \hat{M} \rightarrow \mathrm{B}(H)$ satisfying $\left(\iota \otimes \hat{\pi}_{t}\right)(W)=\tilde{u}_{t} W$.

On the other hand, we have analogously,

$$
\left(\Delta^{\mathrm{op}} \otimes \iota\right)\left(\tilde{w}_{t} \Sigma V^{*} \Sigma\right)=\left(\tilde{w}_{t} \Sigma V^{*} \Sigma\right)_{13}\left(\tilde{w}_{t} \Sigma V^{*} \Sigma\right)_{23} \quad \text { where } \quad \tilde{w}_{t}=(\hat{J} \otimes J) w_{t}^{*}(\hat{J} \otimes J) .
$$

So, $\tilde{w}_{t} \Sigma V^{*} \Sigma$ is a corepresentation of $\left(M, \Delta^{\mathrm{op}}\right)$ which is covariant with respect to the standard representation of $\left(M, \Delta^{\mathrm{op}}\right)^{\wedge}=\hat{M}^{\prime}$. For the same reason as above, we find faithful, normal *-homomorphisms $\hat{\pi}_{t}^{\prime}: \hat{M}^{\prime} \rightarrow \mathrm{B}(H)$ satisfying $\left(\iota \otimes \hat{\pi}_{t}^{\prime}\right)\left(\Sigma V^{*} \Sigma\right)=\tilde{w}_{t} \Sigma V^{*} \Sigma$.

Applying Lemma 10.4 to the actions $\alpha$ and $\beta$ (with $\mathrm{m}=\iota$ ), we find that

$$
(\iota \otimes \Delta)\left(w_{t}\right)\left(1 \otimes u_{t}\right)=(\sigma \otimes \iota)\left(\left(\iota \otimes \Delta^{\mathrm{op}}\right)\left(u_{t}\right)\left(1 \otimes w_{t}\right)\right),
$$

which can be rewritten as

$$
\left(\Sigma V^{*} \Sigma w_{t}\right)_{13} \text { and }\left(W u_{t}\right)_{23} \text { commute. }
$$

This means that the ranges of $\hat{\pi}_{t}$ and $\hat{\pi}_{t}^{\prime}$ commute.

Write $\pi(x)=x$ for $x \in M$. Define $\mathcal{U}_{t}:=\left(\hat{\pi}_{t}^{\prime} \otimes \iota\right)\left(V^{*}\right)(\pi \otimes \iota)\left(W^{*}\right)\left(\hat{\pi}_{t} \otimes \iota\right)(\tilde{V})$ with $\tilde{V}=(J \otimes J) \hat{W}^{*}(J \otimes J)$. Checking it on a slice of $W$, we get that $\left(\hat{\pi}_{t} \otimes \iota\right) \hat{\Delta}^{\mathrm{op}}(x)=(\pi \otimes \iota)(W)\left(\hat{\pi}_{t}(x) \otimes 1\right)(\pi \otimes \iota)\left(W^{*}\right)$ for all $x \in \hat{M}$. So,

$$
(\pi \otimes \iota)\left(W^{*}\right)\left(\hat{\pi}_{t} \otimes \iota\right)(\tilde{V})(1 \otimes a)=\left(\hat{\pi}_{t}(a) \otimes 1\right)(\pi \otimes \iota)\left(W^{*}\right)\left(\hat{\pi}_{t} \otimes \iota\right)(\tilde{V}) \text { for all } a \in \hat{M}
$$

So, we arrive at

$$
\mathcal{U}_{t}(1 \otimes a) \mathcal{U}_{t}^{*}=\hat{\pi}_{t}(a) \otimes 1 \quad \text { for } \quad a \in \hat{M}, \quad \mathcal{U}_{t}(1 \otimes b) \mathcal{U}_{t}^{*}=\pi(b) \otimes 1 \quad \text { for } \quad b \in M,
$$

where the second formula is checked in an analogous way as the first one. Because $\hat{M} M$ is strongly dense in $\mathrm{B}(H)$, it follows that there exist normal, faithful, *-homomorphisms $\Psi_{t}: \mathrm{B}(H) \rightarrow \mathrm{B}(H)$ such that $\mathcal{U}_{t}(1 \otimes x) \mathcal{U}_{t}^{*}=\Psi_{t}(x) \otimes 1$ for all $x \in \mathrm{B}(H)$. It is also clear that the map $t \mapsto \Psi_{t}(x)$ is strong*-continuous for all $x \in \mathrm{B}(H)$.

We prove that $\Psi_{t}(\mathrm{~B}(H))=\mathrm{B}(H)$. So, suppose that $x \in \Psi_{t}(\mathrm{~B}(H))^{\prime}$. Because $\Psi_{t}(a)=a$ for $a \in M$, it follows that $x \in M^{\prime}$ and we write $y=J x J \in M$. Further, $x \in \hat{\pi}_{t}(\hat{M})^{\prime}$, which means that $1 \otimes x$ commutes with $\tilde{u}_{t} W$. Hence, $1 \otimes y$ commutes with $W u_{t}$. So,

$$
\Delta(y)=u_{t}(1 \otimes y) u_{t}^{*}
$$

From this, we conclude that $\Delta^{\mathrm{op}}(y) \in M \otimes N$ and obviously $\left(\Delta^{\mathrm{op}} \otimes \iota\right) \Delta^{\mathrm{op}}(y)=(\iota \otimes \beta) \Delta^{\mathrm{op}}(y)$. This gives us that $\Delta^{\mathrm{op}}(y) \in \beta(N)$ and hence, $y \in N$. So,

$$
\alpha(y)=u_{t}(1 \otimes y) u_{t}^{*}=\tilde{\nabla}^{i t}\left(1 \otimes \sigma_{-t}^{\theta}(y)\right) \tilde{\nabla}^{-i t},
$$

from which we conclude that $\Delta\left(\sigma_{-t}^{\theta}(y)\right)=1 \otimes \sigma_{-t}^{\theta}(y)$. We finally find that $\sigma_{-t}^{\theta}(y) \in \mathbb{C}$ and so, $y \in \mathbb{C}$. This proves that $\Psi_{t}(\mathrm{~B}(H))=\mathrm{B}(H)$.

So, $\Psi_{t}$ is a family of isomorphisms of $\mathrm{B}(H)$ which is pointwise strong*-continuous. It is then easy to find a strongly continuous family $\left(\tilde{v}_{t}\right)$ of unitaries in $\mathrm{B}(H)$ such that $\Psi_{t}=\operatorname{Ad} \tilde{v}_{t}$ for all $t \in \mathbb{R}$. Because $\Psi_{t}(a)=a$ 
for all $a \in M$, we get $\tilde{v}_{t} \in M^{\prime}$. We define $v_{t}:=J \tilde{v}_{t}^{*} J$ and conclude from the equation $\left(\iota \otimes \hat{\pi}_{t}\right)(W)=\tilde{u}_{t} W$ that

$$
u_{t}^{*} W^{*}=\left(1 \otimes v_{t}^{*}\right) W^{*}\left(1 \otimes v_{t}\right) \quad \text { and hence, } \quad u_{t}=\Delta\left(v_{t}^{*}\right)\left(1 \otimes v_{t}\right) .
$$

But then, we observe that $\Delta\left(v_{t}\right) \in N \otimes M$, because $u_{t} \in N \otimes N$, and the same reasoning as above yields $v_{t} \in N$ for all $t \in \mathbb{R}$. So, we write $u_{t}=\alpha\left(v_{t}^{*}\right)\left(1 \otimes v_{t}\right)$.

Because $u_{t}=\left[D \tilde{\tilde{\theta}}: D\left(\operatorname{Tr}_{\hat{\nabla}} \otimes \theta\right)\right]_{t}$, we know that $u_{t+s}=u_{t}\left(\tau_{t} \otimes \sigma_{t}^{\theta}\right)\left(u_{s}\right)$. So, we can calculate

$$
\begin{aligned}
\alpha\left(v_{t+s}^{*}\right)\left(1 \otimes v_{t+s}\right) & =u_{t+s}=u_{t}\left(\tau_{t} \otimes \sigma_{t}^{\theta}\right)\left(\alpha\left(v_{s}^{*}\right)\left(1 \otimes v_{s}\right)\right)=\tilde{\nabla}^{i t}\left(\hat{\nabla}^{-i t} \otimes \nabla_{\theta}^{-i t}\right)\left(\tau_{t} \otimes \sigma_{t}^{\theta}\right)\left(\alpha\left(v_{s}^{*}\right)\left(1 \otimes v_{s}\right)\right) \\
& =\tilde{\nabla}^{i t} \alpha\left(v_{s}^{*}\right)\left(1 \otimes v_{s}\right)\left(\hat{\nabla}^{-i t} \otimes \nabla_{\theta}^{-i t}\right)=\alpha\left(\sigma_{t}^{\theta}\left(v_{s}^{*}\right)\right) u_{t}\left(1 \otimes \sigma_{t}^{\theta}\left(v_{s}\right)\right) \\
& =\alpha\left(\sigma_{t}^{\theta}\left(v_{s}^{*}\right) v_{t}^{*}\right)\left(1 \otimes v_{t} \sigma_{t}^{\theta}\left(v_{s}\right)\right) .
\end{aligned}
$$

It follows that $v_{t} \sigma_{t}^{\theta}\left(v_{s}\right) v_{t+s}^{*}$ is invariant under $\Delta$ and hence, a scalar. We find a continuous function $\lambda: \mathbb{R}^{2} \rightarrow \mathbb{T}$ such that $v_{t+s}=\lambda(t, s) v_{t} \sigma_{t}^{\theta}\left(v_{s}\right)$. It is then easy to check that $\lambda$ is a 2-cocycle and so, necessarily, a 2-coboundary. This means that there exists a continuous function $\mu: \mathbb{R} \rightarrow \mathbb{T}$ such that $\lambda(t, s)=\mu(t) \mu(s) \overline{\mu(t+s)}$. If we replace $v_{t}$ by $\mu(t) v_{t}$, we still have $u_{t}=\alpha\left(v_{t}^{*}\right)\left(1 \otimes v_{t}\right)$ and we get moreover that $v_{t+s}=v_{t} \sigma_{t}^{\theta}\left(v_{s}\right)$ for all $s, t \in \mathbb{R}$. Because the map $t \mapsto v_{t}$ is strong*-continuous, it follows that $\left(v_{t}\right)$ is a $\left(\sigma_{t}^{\theta}\right)$-cocycle.

So, there exists a unique n.s.f. weight $\varphi_{N}$ on $N$ such that $\left[D \varphi_{N}: D \theta\right]_{t}=v_{t}$. Then,

$$
\begin{aligned}
{\left[D \tilde{\tilde{\varphi}}_{N}: D\left(\operatorname{Tr}_{\hat{\nabla}} \otimes \varphi_{N}\right)\right]_{t} } & =\left[D \tilde{\tilde{\varphi}}_{N}: D \tilde{\tilde{\theta}}\right]_{t}\left[D \tilde{\tilde{\theta}}: D\left(\operatorname{Tr}_{\hat{\nabla}} \otimes \theta\right)\right]_{t}\left[D\left(\operatorname{Tr}_{\hat{\nabla}} \otimes \theta\right): D\left(\operatorname{Tr}_{\hat{\nabla}} \otimes \varphi_{N}\right)\right]_{t} \\
& =\alpha\left(v_{t}\right) u_{t}\left(1 \otimes v_{t}^{*}\right)=1 .
\end{aligned}
$$

So, $\left[D \varphi_{N} \circ \alpha: D \varphi_{N}\right]_{t}=1$, which means that $\left(\iota \otimes \varphi_{N}\right) \alpha(x)=\varphi_{N}(x) 1$ for all $x \in \mathcal{M}_{\varphi_{N}}^{+}$. Hence, $\varphi_{N}$ is a left invariant weight on $\left(N,\left.\Delta\right|_{N}\right)$.

Because $R(N)=N$, we can restrict $R$ to $N$ and we find an anti-automorphism of $N$ that anti-commutes with $\left.\Delta\right|_{N}$. So, we also get a right invariant weight $\left.\varphi\right|_{N} R$ and $\left(N,\left.\Delta\right|_{N}\right)$ is a l.c. quantum group.

\section{References}

[1] S. BAAJ, Représentation régulière du groupe quantique des déplacements de Woronowicz. Astérisque 232 (1995), 11-48.

[2] S. BaAJ \& G. Skandalis, Unitaires multiplicatifs et dualité pour les produits croisés de $\mathrm{C}^{*}$-algèbres. Ann. Scient. Ec. Norm. Sup. (4) 26 (1993), 425-488.

[3] S. BaAJ, G. Skandalis \& S. Vaes, Non-semi-regular quantum groups coming from number theory. Comm. Math. Phys., to appear.

[4] V. G. Drinfel'D, Quantum groups. In Proceedings ICM (Berkeley, Calif., 1986), Amer. Math. Soc., Providence, RI, 1987, pp. $798-820$.

[5] M. Enock \& J-M. Schwartz, Kac Algebras and Duality of Locally Compact Groups, SpringerVerlag, 1992.

[6] U. HaAgerup, Operator valued weights in von Neumann algebras I. J. Funct. Anal. 32 (1979), 175206.

[7] J. Kustermans, Locally compact quantum groups in the universal setting. Int. J. Math. 12 (2001), 289-338.

[8] J. Kustermans \& S. Vaes, Locally compact quantum groups. Ann. Scient. Ec. Norm. Sup. (4) 33 (2000), 837-934. 
[9] J. Kustermans \& S. VAes, Locally compact quantum groups in the von Neumann algebraic setting. Math. Scand., to appear.

[10] S. MaJID, More examples of bicrossproduct and double cross product Hopf algebras. Isr. J. Math. 72 (1990), 133-148.

[11] T. Masuda \& Y. NAKagami, A von Neumann algebraic framework for the duality of the quantum groups. Publ. RIMS, Kyoto University 30 (1994), 799-850.

[12] S. VAes, Strictly outer actions of locally compact groups and quantum groups. Preprint.

[13] S. VAES, The unitary implementation of a locally compact quantum group action. J. Func. Anal. 180 (2001), 426-480.

[14] S. VAes, A Radon-Nikodym theorem for von Neumann algebras. J. Operator Theory 46 (3) (2001), $477-489$.

[15] S. VAes \& L. VAinerman, Extensions of locally compact quantum groups and the bicrossed product construction. Adv. in Math., to appear.

[16] S. Vaes \& A. VAn Daele, The Heisenberg commutation relations, commuting squares and the Haar measure on locally compact quantum groups. Proceedings of the OAMP Conference, Constantza, 2001, to appear.

[17] A. Van Daele, An algebraic framework for group duality. Adv. in Math. 140 (1998), 323-366.

[18] S.L. Woronowicz, Compact matrix pseudogroups. Comm. Math. Phys. 111 (4) (1987), 613-665.

[19] S.L. Woronowicz, Compact quantum groups. In Symétries quantiques (Les Houches, 1995), NorthHolland, Amsterdam, 1998, pp. 845-884.

[20] S.L. Woronowicz, From multiplicative unitaries to quantum groups. Int. J. Math. 7 (1) (1996), $127-149$.

[21] T. Yamanouchi, Takesaki duality for weights on locally compact quantum group covariant systems. J. Operator Theory, to appear.

[22] T. Yamanouchi, Canonical extension of actions of locally compact quantum groups. J. Func. Anal., to appear.

[23] T. Yamanouchi, Double group construction of quantum groups in the von Neumann algebra framework. J. Math. Soc. Japan 52 (4) (2000), 807-834. 